2017-12

\title{
Dementia prevention, intervention, and
} care

\author{
Livingston, $\mathrm{G}$
}

http://hdl.handle.net/10026.1/18238

10.1016/s0140-6736(17)31363-6

The Lancet

Elsevier BV

All content in PEARL is protected by copyright law. Author manuscripts are made available in accordance with publisher policies. Please cite only the published version using the details provided on the item record or document. In the absence of an open licence (e.g. Creative Commons), permissions for further reuse of content should be sought from the publisher or author. 


\section{The Lancet International Commission on Dementia Prevention and}

Care

Professor Gill Livingston MD *

Division of Psychiatry, University College London, UK; Camden and Islington NHS Foundation Trust, London, UK

Andrew Sommerlad MSc

Division of Psychiatry, University College London, UK

Vasiliki Orgeta PhD

Division of Psychiatry, University College London, UK

Sergi G Costafreda PhD

Division of Psychiatry, University College London, UK; Camden and Islington NHS Foundation Trust, London, UK

Jonathan Huntley PhD

Division of Psychiatry, University College London, UK; Department of Old Age Psychiatry, King's College

London, UK

Professor David Ames MD

National Ageing Research Institute and University of Melbourne Academic Unit for Psychiatry of Old Age,

Parkville and Kew, Victoria, Australia

Professor Clive Ballard MD

Dean of Medicine, University of Exeter, UK

Professor Sube Banerjee MD

Centre for Dementia Studies, Brighton and Sussex Medical School, University of Sussex, Brighton, East Sussex, UK

Professor Alistair Burns MD

University of Manchester, Manchester, UK

Professor Jiska Cohen-Mansfield PhD

Department of Health Promotion, School of Public Health, Sackler Faculty of Medicine, Tel Aviv University, Tel Aviv, Israel; Heczeg Institute on Aging, Tel Aviv University, Tel Aviv, Israel; Minerva Center for Interdisciplinary Study of End of Life, Tel Aviv University, Tel Aviv, Israel

Claudia Cooper PhD

Division of Psychiatry, University College London, UK; Camden and Islington NHS Foundation Trust, London, UK

Professor Nick Fox MD

Dementia Research Centre, University College London, Institute of Neurology, National Hospital for Neurology and Neurosurgery, London, UK

Laura N Gitlin PhD

Center for Innovative Care in Aging, Johns Hopkins University, Baltimore, Maryland, USA

Professor Robert Howard MD

Division of Psychiatry, University College London, UK; Camden and Islington NHS Foundation Trust, London, UK 
Professor Helen C Kales MD

Department of Psychiatry, University of Michigan, Ann Arbor, MI, USA and VA Center for Clinical Management Research, Ann Arbor, MI, USA

Professor Eric Larson MD

Group Health Research Institute, Group Health Cooperative, Seattle, WA, USA

Professor Karen Ritchie PhD

Inserm, Unit 1061, Neuropsychiatry: Epidemiological and Clinical Research, La Colombière Hospital, University of Montpellier, France; Faculty of Medicine, Imperial College, London, UK

Professor Kenneth Rockwood MD

Centre for the Health Care of Elderly People, Geriatric Medicine Dalhousie University, Halifax, Nova Scotia, Canada

\section{Elizabeth L Sampson MD}

Marie Curie Palliative Care Research Department, Division of Psychiatry University College London, London, UK

Quincy Samus PhD

Department of Psychiatry and Behavioral Sciences, Johns Hopkins Bayview, Johns Hopkins University, Baltimore, MD, USA

Professor Lon S Schneider MD

Department of Psychiatry and the Behavioural Sciences and Department of Neurology, Keck School of Medicine, Leonard Davis School of Gerontology of the University of Southern California, Los Angeles, CA, USA

Professor Geir Selbæk PhD

Norwegian National Advisory Unit on Aging and Health, Vestfold Health Trust, Tønsberg, Norway; Institute of Health and Society, Faculty of Medicine, University of Oslo, Oslo, Norway; Centre for Old Age Psychiatric Research, Innlandet Hospital Trust, Ottestad, Norway

Professor Linda Teri PhD

Department Psychosocial and Community Health, School of Nursing, University of Washington, Seattle, USA

Naaheed Mukadam MSc

Division of Psychiatry, University College London, UK

* Corresponding author 


\section{Executive Summary}

\section{Acting now on dementia prevention and care can vastly improve living and dying for individuals with dementia and their families and in doing so transform the future for society.}

Dementia is the greatest global challenge for health and social care in the 21st century. It occurs mainly in older people so increases in numbers and costs are driven, worldwide, by increased longevity resulting from the welcome reduction in people dying prematurely. The Lancet International Commission on Dementia Care met to consolidate the huge strides that have been made and the emerging knowledge as to what we should do to prevent and manage dementia.

There were around 47 million people living with dementia globally in 2015, and this is projected to increase to 66 million by 2030 and 115 million by 2050. Dementia affects the individual living with it, who gradually loses abilities, as well as their relatives and other supporters, who have to cope with seeing a family member or friend become ill and decline, while responding to their needs, such as increasing dependency and changes in behaviour. In addition, it affects wider society as people also require health and social care. The 2015 global cost of dementia was estimated to be US\$818 billion and this will continue to increase as the numbers of people with dementia rise. Nearly $85 \%$ of costs are related to family and social, rather than medical, care. It may be that future new medical care, including public health measures, may replace and possibly reduce some of this cost.

Dementia is by no means an inevitable consequence of reaching retirement age, or even of entering the ninth decade. There are lifestyle factors that may reduce, or increase, an individual's risk of developing dementia. In some populations dementia is already being delayed for years; while in others the numbers of people living with it has increased. In this commission, we have extended current models of risk by including hearing loss, derived from a new review and meta-analysis performed for this report, and social isolation. Incorporating potentially reversible risk factors from different phases of the life-span and not just old age, we are able to propose a novel life-course model of risk from which population attributable fractions have been derived to demonstrate the possible impact on future incidence of successful elimination of the most potent factors. We have brought together all this evidence and have calculated that around one third of dementia may theoretically be preventable. More childhood education, exercise, maintaining social engagement, reducing or stopping smoking, management of hearing loss, depression, diabetes, hypertension and obesity could all contribute to prevention or delay of dementia. There is also preliminary evidence about other potentially modifiable risk factors. We have outlined the mechanisms by which these risk factors impact on the brain.

Of course not everyone will be able to make changes, some changes will not make a difference and some dementia risk is genetic and not currently modifiable. Nonetheless delaying dementia for some years for even a small percentage of people would be an enormous achievement and enable many more people to reach the end-of-life without developing dementia. Many people present to services with Mild Cognitive Impairment $(\mathrm{MCl})$ a risk state for dementia, which occurs in up to a fifth of people aged over 65 and this provides an opportunity for more targeted interventions.

It is now known that many of dementia's manifestations are manageable and while the underlying dementing illnesses are generally not curable, they may be modifiable with good dementia care. In 
this report, we have summarised what should be done now, and when the available evidence is not definitive, we have made this clear.

We have itemised interventions which can transform the lives of people with dementia and their families, maximising cognition, decreasing distressing associated symptoms, reducing crises and improving quality of life. Timely diagnosis is a prerequisite to receiving these interventions. We are interested in what works and have included pharmacological, psychological, environmental and social interventions. If these are implemented, people with dementia will have their cognition optimised and be less likely to be agitated, depressed or have troublesome psychotic symptoms and family carers will have reduced levels of anxiety and depression. It is also important to discuss future decision-making as soon as possible with people with dementia and allow them to nominate an agent who can enact pre-specified wishes or make choices consistent with their values.

People with dementia are usually older, often have co-morbidities and may need help in coping with these illnesses. A third of older people now die with dementia and all professionals working in endof-life care need to make this a central part of their planning and communication.

In this commission, we have detailed evidence-based approaches to dementia and its symptoms. Services should be available, scalable and give value. As there are limited resources, professionals and services need to use what works, not use what is ineffective, and be aware of the difference.

Overall, there is good potential for prevention and, once someone develops dementia, for care to be high-quality, accessible, and give value to an under-served, growing population. Effective dementia prevention and care could transform the future for society and vastly improve living and dying for individuals with dementia and their families. Acting now on what we already know can make this difference happen. 


\section{Key Messages}

1 There are increasing numbers of people with dementia globally although incidence in some countries has decreased.

2 Be ambitious about prevention: We recommend energetically treating hypertension in middle aged and older people without dementia to reduce dementia incidence. Interventions for other risk factors, including more childhood education, exercise, maintaining social engagement, reducing smoking, and management of hearing loss, depression, diabetes and obesity; may have the potential of delaying or preventing a third of dementias.

3 Treat cognitive symptoms: To maximise cognition, people with Alzheimer's dementia or Dementia with Lewy Bodies should be offered Cholinesterase Inhibitors (ChEls)at all stages, or memantine for severe dementia. ChEls are not effective in Mild Cognitive Impairment.

4 Individualise dementia care: Good dementia care spans medical, social and supportive care, should be tailored to unique individual and cultural needs, preferences, priorities, and should incorporate support for the family carers

5 Care for family carers. Family carers are at high risk of depression. Effective interventions reduce the risk and treat the symptoms, include START (Strategies for Relatives) or REACH (Resources for Enhancing Alzheimer's Caregiver Health intervention) and should be made available.

6 Plan for the future. People with dementia and their families value discussions about the future and decisions about possible attorneys to make decisions. Clinicians should consider capacity to make different types of decisions at diagnosis.

7 Protect people with dementia. People with dementia and society require protection from possible risks of the condition, including self-neglect, vulnerability including to exploitation, managing money, driving or using weapons. Risk assessment and management at all stages of the disease is essential but it should be balanced against the persons' right to autonomy. 8 Manage neuropsychiatric symptoms. Management of the neuropsychiatric symptoms of dementia including agitation, low mood or psychosis, is usually psychological, social, and environmental, with pharmacological management reserved for those with more severe symptoms.

9 Consider end of life. A third of older people die with dementia, so it is essential that professionals working in end-of- life care consider whether a patient has dementia as they may be unable to make decisions about their care and treatment or express their needs and wishes.

10 Technology: Technological interventions have the potential to improve care delivery but should not replace social contact.

\section{Introduction}

As the world's population ages, increasing numbers of people are living with dementia and this is projected to continue to rise, especially in low- and middle-income countries (figure 1) (1). There were around 47 million people living with dementia worldwide in 2015 , affecting the individual living 
with it, their family as they become more dependent and the wider society which provides and often pays for care and support. The annual global cost of dementia is estimated to be US $\$ 818$ billion (2). Nearly $85 \%$ of costs are related to family and social, rather than medical, care. Future medical care, including public health measures, may replace and reduce some of this cost (3).The number of people with dementia is expected to increase to 66 million by 2030 and 115 million by 2050 (4), driven by rising numbers of older adults $(5 ; 6)$. However, some recent population studies have found a lower incidence of people with dementia than predicted from previous projections, and therefore while the rate of increase and crisis related to providing care continues, this may not be quite as large as previously expected $(7 ; 8)$.

Dementia was long considered to be neither preventable nor treatable, but encouraging progress has been made. The Lancet International Commission on Dementia Prevention and Care has therefore met to consolidate emerging knowledge as to what can work and what individuals should do to prevent and manage dementia particularly with the health systems in high income countries. It is now known that many of dementia's manifestations are manageable and while the underlying illnesses are not curable, the course may be modifiable with good dementia care. Current care can improve the trajectory of symptoms and the family's ability to cope with them and thus change the experience of the course of dementia. Additionally, there is evidence that an important fraction of dementia is preventable.

Dementia and Mild Cognitive Impairment $(\mathrm{MCl})$ are characterised by decline from a previously attained cognitive level but in dementia, in contrast to $\mathrm{MCl}$, the decline impacts on activities of daily living or social functioning (9). In MCl, although the patient can still engage in complex activities, for example, paying bills or taking medication, greater effort or new strategies may be required.

Dementia is usually preceded by $\mathrm{MCl}$, and the boundary between two is grey, and many people present to dementia services with $\mathrm{MCl}$.

There are many different causes of dementia, with Alzheimer's disease (AD) the most common. Vascular dementia is the next most common, followed by dementia associated with Lewy bodies (DLB). Mixed dementia with features of more than one aetiology are also common. Frontotemporal degeneration and dementias associated with brain injury, infections, and alcohol abuse are less common causes (10). In this commission when we use the word dementia we are referring to all the different types of dementia.

The word "dementia" is derived, from the Latin 'De' - out of and 'Mens'- mind and its use has been considered by some to have demeaning connotations. There are stigmatising cultural beliefs about dementia, such as it is a punishment or a curse (11). This may lead to people avoiding diagnosis as they may feel stigmatised by others or in their own mind. Diagnostic and Statistical Manual of Mental Disorders (DSM 5) has stopped using the word dementia and instead uses the phrase "major neurocognitive disorders" (12). These are illnesses with demonstrable neural substrate abnormalities together with cognitive symptoms, which occur in people who have had normal brain development (13). Mild neurocognitive disorder has also been added to DSM 5, equating to World Health Organisation International Classification of Diseases (ICD-10) classification of mild cognitive disorder (9).

The evaluation of the needs of a person with dementia has to consider other illnesses and medications that impact and interact with the dementia, and their social and physical living 
environment. Dementia usually occurs in people aged $>65$ years old (14) when comorbidity is common. Age-related physical health problems and dementia co-occur more often than by chance. This is because some physical problems, such as diabetes and hypertension, increase the risk of Alzheimer's disease and vascular dementia, making a mixed dementia more likely; and the more physical illnesses a person has, the more likely they are to develop dementia, possibly related to a lack of resilience and repair contributing to all of these problems (15). Impaired mental and physical function also interfere with exercise or social activities (16). These health and social challenges influence diagnosis, prognosis, response to treatment and need for health and social care. Yet people with complex needs are generally under-represented in trials; those who are eligible for and participate in research tend to be fitter, younger, male and more highly educated (17).

In this commission we have used the best current evidence to make recommendations. When current evidence is incomplete we have summarised the balance of evidence and explained its strengths and limitations. An overall limitation is that this is generally focussed on and from high income countries and we have less evidence from middle and low income countries.

\section{Preventing}

\section{Demographics and dementia}

The number of people with dementia is rising rapidly (figure 1), primarily due to worldwide ageing populations, particularly in low- and middle-income countries $(1 ; 4)$. This trend is expected and widely reported $(18 ; 19)$. There are increasing numbers of people with dementia everywhere.

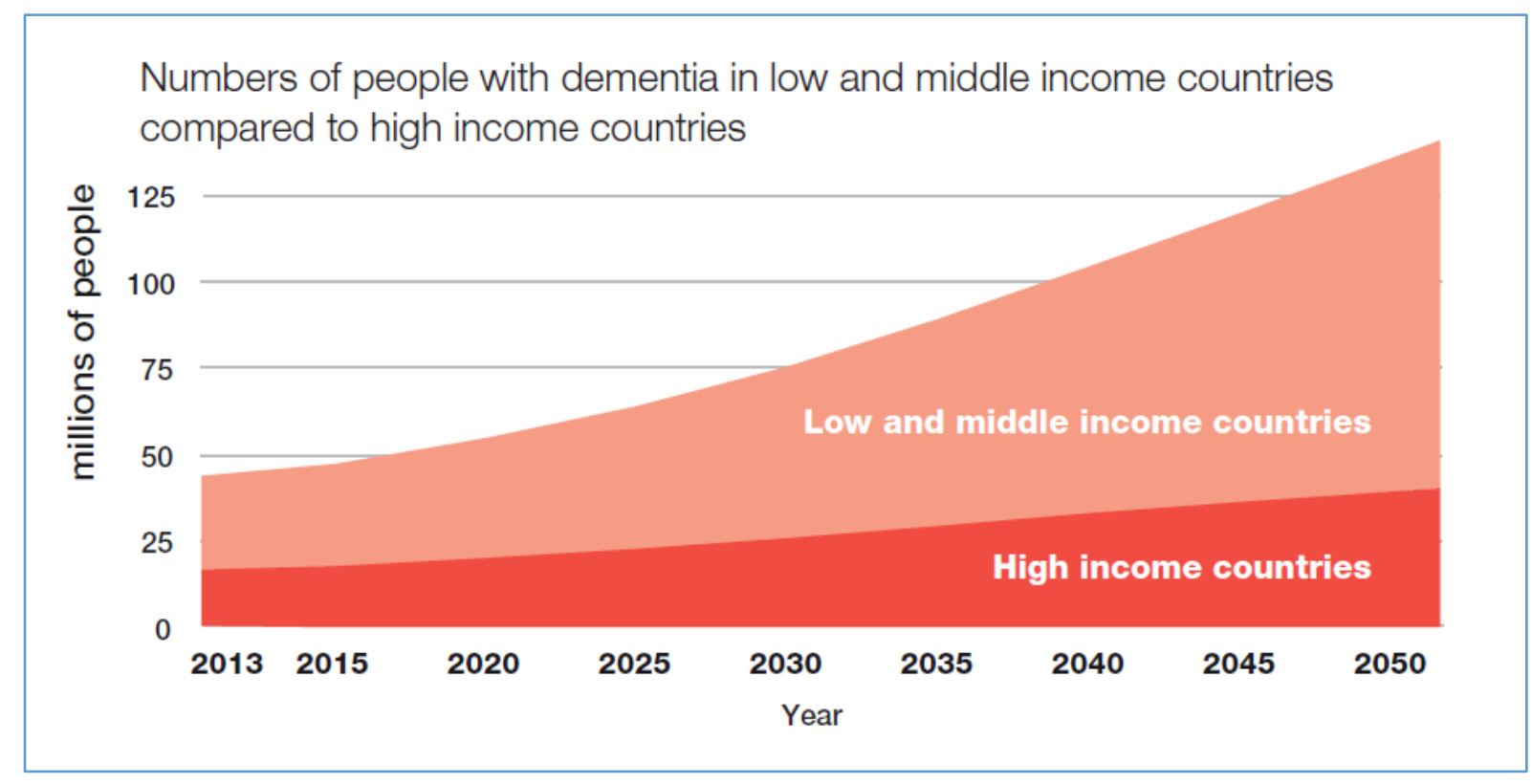

Figure 1. The growth in numbers of people with dementia in high-income and low- and middleincome countries 
Figure reproduced from 'The Global Impact of Dementia 2013-2050' (1) by permission of Alzheimer's Disease International

While there is no current disease-modifying treatment for any common dementia, delaying dementia's onset would benefit even the oldest adults (20). There has been an unexpected observed decline in age-specific dementia incidence or prevalence rates in some countries, such as the United States (US), the United Kingdom (UK), Sweden, the Netherlands, and Canada (7;8;21-26). Conversely, there have been reports of an increase in incidence rates in China (27) and prevalence rates in Japan $(28 ; 29)$ while rates in Nigeria are stable (30).Both US studies found the decrease in age specific prevalence (despite an increase in the absolute number of people with dementia) was associated with increasing education $(25 ; 26)$.

These data suggest reduced dementia risk in successive generations according to their lifetime exposure to health and lifestyle factors. In some countries, the current cohort of people aged over 65 is cognitively healthier than their predecessors with greater resilience, as a result of reduced exposure to dementia risk factors, or increased exposure to protective factors. However, the increasing midlife rates of obesity and associated ill-health are projected to lead to a $19 \%$ increase in dementia rates in China and $9 \%$ in US (31).

\section{The complexity of dementia neuropathology complicates prevention}

Some dementia risk factors, including cardiovascular, cerebrovascular, metabolic and psychiatric factors, diet, lifestyle and education, are potentially changeable (32). Dementia is heterogeneous and risk factors vary, and also co-exist, for different types of dementia. Vascular brain injury including strokes and microvascular infarcts not only lead to vascular dementia but occur more commonly in older people with AD (33-35), and are present in some people who do not have dementia. In those with both neuropathological $A D$ and lacunar infarcts, the cognitive impairment is more severe than those without such infarcts (36). These patients are sometimes diagnosed as having "mixed dementia" (37), AD in which plaques and tangles are seen alongside microvascular infarcts or less commonly Lewy bodies, all of which likely contribute to cognitive decline (38-42).

It is possible, as we have done below, to model the effect of changing the potentially modifiable risk factors. Currently, the evidence for lifestyle changes on cognitive decline is mixed. The changes in incidence reported in diverse countries provide evidence that reducing or increasing rates of dementia are both possible. Lower rates indicate either that onset has been delayed for some people or that other competing causes of mortality occurred (43).

The EU Joint Programme on Neurodegenerative Disease Research called in 2014 for population- and disease-based cohorts to be exploited to obtain the high quality evidence necessary to capture the range of potential health influences and confounding factors starting in midlife, and provide evidence on the direction of causality(44).

While modifying risk factors is important in dementia prevention, age, the greatest risk factor for dementia overall, is unmodifiable. Dementia usually presents in older age, with exponential increases in incidence over the age of 65 . Overall about $80 \%$ of dementias are in people aged $\geq 75$ 
years $(14 ; 45)$ and there may be an interaction between age, neuropathology, comorbidity and the clinical presentation. It is likely that age on its own would be a less powerful risk factor once other risk factors and comorbidity are taken into account but it still remains an important consideration, especially as life expectancy continues to increase.

\section{A focus on resilience: cognitive reserve}

Some people with neuropathological changes of AD do not have dementia (40), indicating resilience. Figure 2 illustrates how some cognitively normal individuals in community-based U.S. studies tolerate a large and mixed burden of vascular, Lewy body and Alzheimer's neuropathology (40). These findings have led to the concept of cognitive reserve, which is that people who have such brain reserve can tolerate more neuropathology without cognitive and functional decline, therefore develop dementia more slowly (46). This reserve is related to either the brain anatomical substrate or adaptability of cognition, due to factors discussed in more detail below $(47 ; 48)$.

The theory suggests that less cognitive reserve leads to earlier development of dementia. Furthermore, it suggests that populations with, for example, increased rates of hypertension might develop dementia earlier, as the resultant neuropathology reduces the cognitive reserve buffer. As predicted, those with less cognitive reserve due to intellectual disability develop dementia at a younger age (49). Additionally, people of African origin in the UK and US who have high rates of hypertension, have increased rates of dementia at younger age (50-52). 


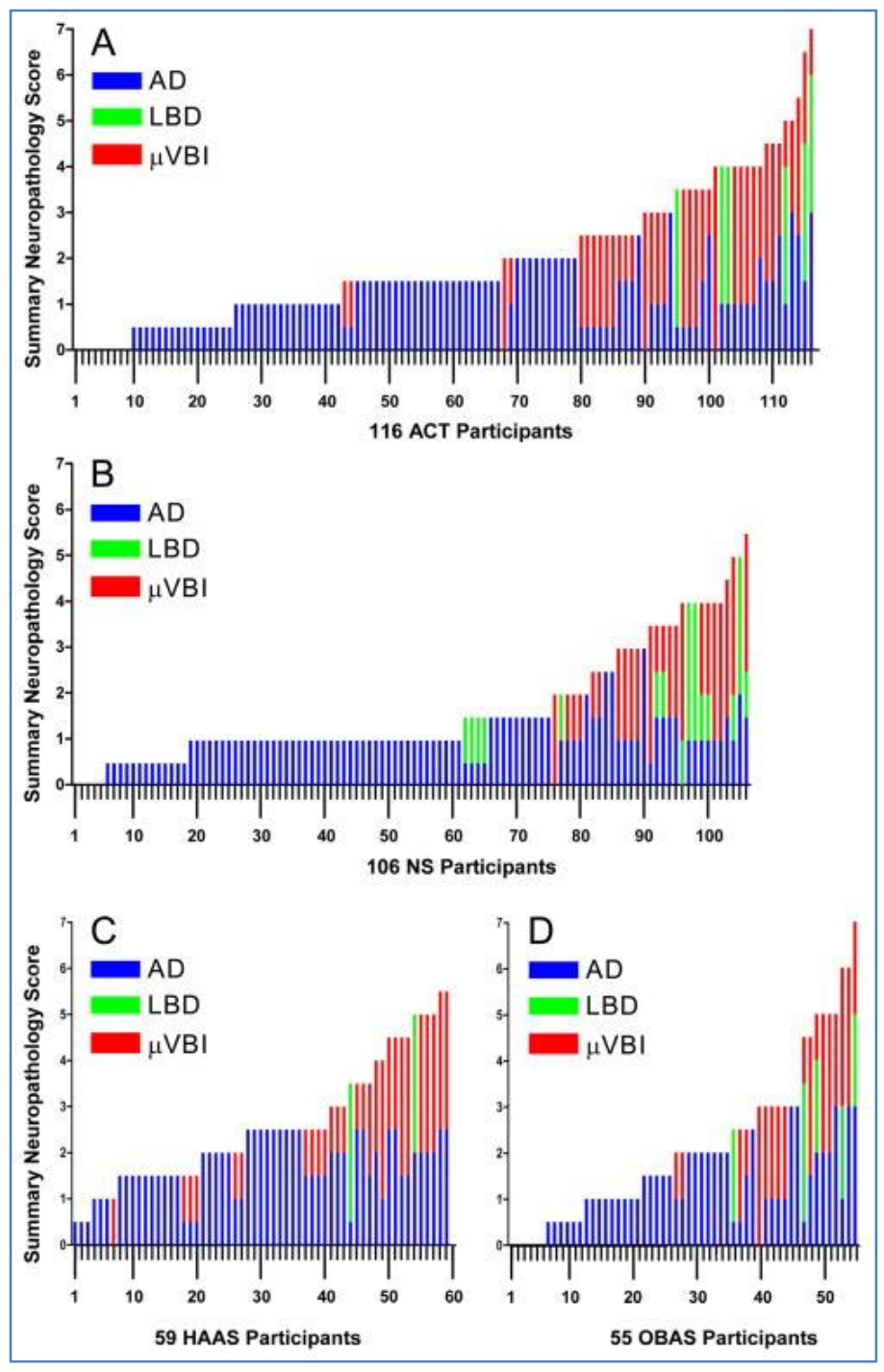

Figure 2. Brain autopsy results from cognitively normal individuals expressed as Summary Neuropathology Score (potentially range 0 to 9) ranked from lowest to highest.

Key: Each stacked bar shows an individual's burden of $A D$ (blue), $L B D$ (green), and $\mu V B I$ (red). (A) 116 Adult Changes in Thought (ACT) participants, (B) 106 Nun Study (NS) participants, (C) 59 Honolulu Asian Aging Study (HAAS) participants, (D) 55 Oregon Brain Health \& Science (OBAS) participants.

Figure reproduced from Sonnen et al (40) by permission of the American Medical Association

A broader approach to dementia prevention including promoting resilience makes sense in our ageing societies. Strategies for promoting resilience to prevent or delay dementia's onset are extrapolated from studies on declining dementia incidence rates which report healthier lifestyles are associated with declining prevalence of cognitive impairment and dementia (23;24). Cognitive resilience in late life is likely to be enhanced by building reserve earlier in life through education and other intellectual stimulation (53;54). Through neuronal branching and plasticity such changes may subsequently be translated into brain reserve. Lower rates of late-life dementia are found with higher education levels (25). Improved socio-economic status during gestation and early childhood has a protective association with late-life dementia risk (55). These findings indicate that improving 
brain reserve $(53 ; 54 ; 56)$ combined with interventions known to prevent damage are ways to promote resilience.

\section{Modifiable Risk Factors for dementia}

Prevention is better than cure and underlies the growing interest in modifiable risk factors. Any future disease-modifying treatment for dementia will not remove the need for effective prevention of dementia. In the risk literature, midlife has been defined as aged 45-65 years and late life as aged $>65$. We have used these definitions throughout this commission for consistency, but these risks are often relevant throughout the life course. Much of this work focuses on estimating the Population Attributable Fraction (PAF), which is the percentage reduction in new cases over a given time if a particular risk factor were completely eliminated. The work to date focuses on wellestablished cardiovascular risk factors for dementia, including diabetes, midlife hypertension, midlife obesity, physical inactivity and smoking, as well as depression and low educational attainment (32).

\section{Newly calculated population attributable fraction for modifiable risk factors}

\section{Which modifiable risk factors?}

We sought to calculate a combined PAF for known modifiable risk factors for dementia. We decided which risk factors to include by identifying those listed in the UK National Institute of Health and Care Excellence (NICE) (57) and US National Institute of Health (NIH) (58) guidelines. For risk factors included in recent papers reporting dementia PAF - vascular risk factors, not continuing in education beyond primary school and depression $(32 ; 59)$ - we used their data on relative risk and prevalence. For the additional risk factors included in our calculations, we sought systematic reviews of their relative risk and prevalence and, in the absence of one; we asked other authors on the Lancet Commission for suitable papers and conducted our own meta-analysis. We focused on all-cause rather than cause-specific dementia as there was most data for this outcome. As far as possible, we used prevalence and relative risk data from international studies to make our figures relevant to global dementia risk (see Table 1).

NICE and NIH identify social isolation and peripheral hearing loss as potentially modifiable dementia risk factors.

We used a systematic review and meta-analysis for social isolation and incident dementia to calculate its PAF (60). This paper divided the exposure into social contact (telephone or face-to-face contact with family or friends), social participation (belonging to or taking part in community activities or organisations) and loneliness (a subjective feeling of dissatisfaction at one's level of social contact). We have used the figures for social contact as we judged this the most accurate measure of actual contact time. The weighted relative risk (RR) for incident dementia associated with less frequent social contact was 1.57 (95\% $\mathrm{Cl}$ [1.32-1.85]). PAF calculations require knowing the prevalence of the risk factor but this was not given in any of these papers. There was also heterogeneity in individual papers' definition of infrequent social contact. We therefore used results from a representative sample of older people in the UK (61) to estimate prevalence and incorporated the prevalence of reporting social contact less than monthly, which is probably a conservative definition. 
There were no systematic reviews for hearing loss and incident dementia. We therefore consulted experts to generate a list of relevant papers and used the quality checklist for prognosis studies (62) defining high quality papers as those that had followed a cohort of cognitively healthy people for at least 5 years, had an objective measure of peripheral hearing (pure-tone audiometry), incident dementia as an outcome, and adjusted for age and cardiovascular risk factors as potential confounding factors. Three studies met these criteria (63-65), with follow-up over 9, 12 and 17 years. Each found that peripheral hearing loss was a significant risk factor for dementia. We metaanalysed this data and calculated a pooled RR of 1.94 (95\% Cl [1.38-2.73]) (figure 3).

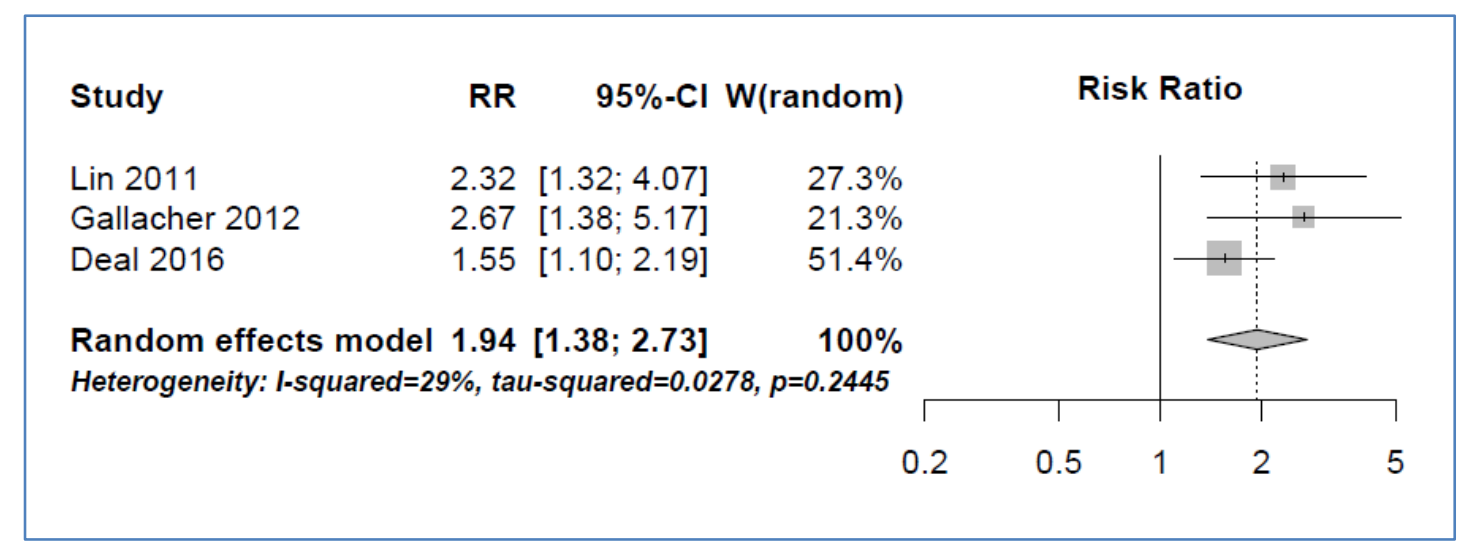

Figure 3. Forest plot of effect of hearing loss, measured by pure tone audiometry, on incident dementia rates $9-17$ years later in cognitively healthy people

\section{PAF for modifiable risk factors}

The attributable risk in a population depends on the prevalence of the risk factor and the strength of its association (relative risk) with the disease. In our calculations, we have used relative risks from systematic reviews and, while these were adjusted for many confounders, they could not have been adjusted for all the risk factors in our total PAF calculation. Therefore using the formula for calculation of individual risk factor PAF for circumstances in which all confounding risk factors have been adjusted for would be inappropriate (66). We therefore used a version of the formula which was used in a previous paper and which is more appropriate when confounding has not been fully accounted for (32;59). 


\section{Formula for individual Population Attributable Fraction (PAF)}

$\mathrm{PAF}=\mathrm{Pe}(\mathrm{RRe}-1) /[1+\mathrm{Pe}(\mathrm{RRe}-1)]$

$\mathrm{Pe}=$ prevalence of the exposure

$\mathrm{RRe}=$ relative risk of disease due to that exposure

\section{Calculation of communality}

- Input data on all nine risk factors in our model

- Calculate tetrachoric correlation to generate correlation coefficients and a correlation matrix.

- Conduct a principal-component analysis on the correlation matrix to generate eigenvectors, which are directions mapped onto the data points and from which variance to the data is measured. These represent unobserved factors underlying all the variables that explain the variance observed.

- Components with eigenvalues $\geq 1$ were retained in the model

- Communality was calculated as the sum of the square of all factor loadings (i.e. how much each unobserved component explained each measured variable).

\section{Calculation of overall Population Attributable Fraction (PAF)}

We then calculated overall PAF: PAF $=1-\left[\left(1-\mathrm{PAF}_{1}\right)\left(1-\mathrm{PAF}_{2}\right)\left(1-\mathrm{PAF}_{3}\right) \ldots\right]$

Each individual risk factor's PAF was weighted according to its communality using the formula:

$$
\text { Weight }(w)=1 \text {-communality }
$$

Weighting was included in the calculation of overall PAF using the formula:

$$
\text { PAF }=1-\left[\left(1-w^{*} P A F_{1}\right)\left(1-w^{*} P^{\prime} F_{2}\right)\left(1-w^{*} P A F_{3}\right) \ldots\right]
$$

\section{Box 1. Method for calculation of population attributable fraction and communality}

\section{Communality of risk factors}

We used figures from the 2014 Health Survey for England (HSE), a representative sample of over 10,000 UK community-dwelling adults, to calculate communality of risk factors - the variance in observed variables accounted for by common factors - to allow calculation of each factor's unique risk $(32 ; 67 ; 68)$. HSE data has all the relevant risk factors except social contact frequency, so we used cohabitation as a proxy measure for social contact, with the assumption that those participants who live with someone else have higher levels of social contact than those who live alone. Our principal component analysis, extracted using this method, found that there were three principal components which explained $53 \%$ of the total variance between the nine risk factors, suggesting substantial overlap. The prevalence, communality and relative risk with the PAF adjusted for communality of each included risk factor is in table 1. We then calculated overall PAF (table 1) using the same formula as others had but incorporating the additional variables of hearing loss and social isolation (box 1). We present the new model of life course risk factors in figure 4. 
Total potentially modifiable risk factors for dementia

\begin{tabular}{lccccc}
\hline \multicolumn{1}{c}{ Risk Factor } & $\begin{array}{c}\text { Relative risk } \\
\text { for dementia }\end{array}$ & $\begin{array}{c}\text { Prevalence } \\
\text { (\%) }\end{array}$ & $\begin{array}{c}\text { Communality } \\
\text { (\%) }\end{array}$ & $\begin{array}{c}\text { PAF } \\
\text { (\%) }\end{array}$ & $\begin{array}{c}\text { Weighted } \\
\text { PAF* (\%) }\end{array}$ \\
\hline \multicolumn{1}{c}{ Early life } & & & & & \\
\hline $\begin{array}{l}\text { Less education (none or primary } \\
\text { school only) }\end{array}$ & 1.6 & 40.0 & 64.6 & 19.1 & $\mathbf{7 . 5}$ \\
\hline \multicolumn{1}{c}{ Midlife (age 45-65) } & & & & & \\
\hline Hypertension & 1.6 & 8.9 & 57.3 & 5.1 & $\mathbf{2 . 0}$ \\
\hline Obesity & 1.6 & 3.4 & 60.4 & 2.0 & $\mathbf{0 . 8}$ \\
\hline Hearing loss & 1.9 & 31.7 & 46.1 & 23.0 & $\mathbf{9 . 1}$ \\
\hline \multicolumn{1}{c}{ Later life (age $>$ 65) } & & & & & \\
\hline Smoking & 1.6 & 27.4 & 51.1 & 13.9 & $\mathbf{5 . 5}$ \\
\hline Depression & 1.9 & 13.2 & 58.6 & 10.1 & $\mathbf{4 . 0}$ \\
\hline Physical inactivity & 1.4 & 17.7 & 26.6 & 6.5 & $\mathbf{2 . 6}$ \\
\hline Low social contact & 1.6 & 11.0 & 45.9 & 5.9 & $\mathbf{2 . 3}$ \\
\hline Diabetes & 1.5 & 6.4 & 70.3 & 3.2 & $\mathbf{1 . 2}$ \\
\hline Total adjusted for communality & & & & & $\mathbf{3 5 . 0}$ \\
\hline
\end{tabular}

Table 1. Risk factors for dementia; relative risk for dementia, prevalence, communality and Population Attributable Fraction (PAF) for dementia

Notes: *Weighted PAF is the relative contribution of each risk factor to the overall PAF when adjusted for communality

Our results suggest that around $35 \%$ of dementia is attributable to a combination of these nine risk factors; namely education to a maximum of age 11 or 12, midlife hypertension, midlife obesity, hearing loss and later-life depression, diabetes, physical inactivity, smoking and social isolation. In comparison, completely eliminating the Apolipoprotein $E(A p o E) ~ \varepsilon 4$ allele as the major genetic risk factor is calculated to produce a 7\% reduction in incidence, using the PAF calculation methods (69). 


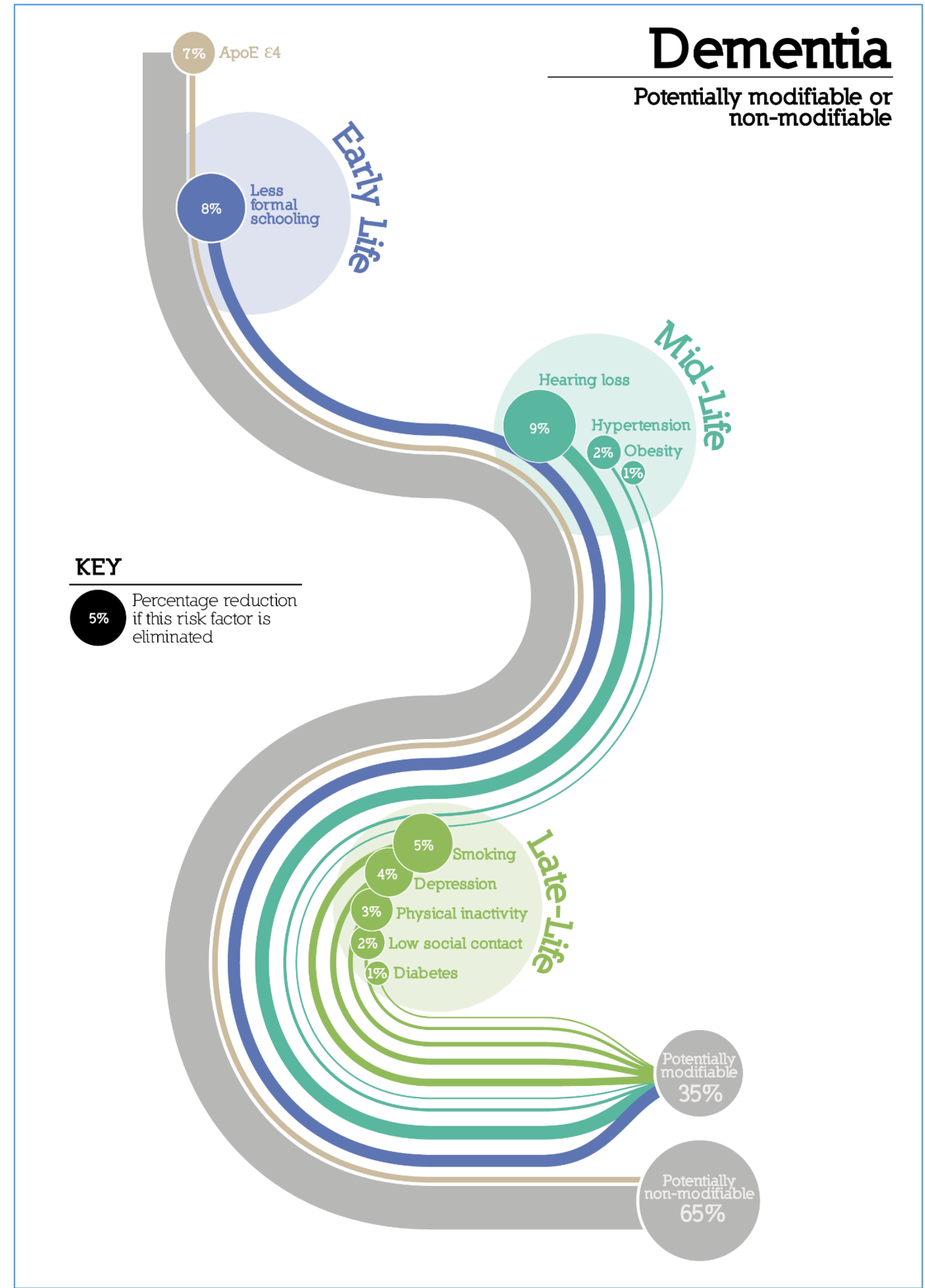

Figure 4. Life course model of contribution of modifiable risk factors to dementia Notes: Numbers are rounded to nearest integer. 


\section{Effects of potentially modifiable risk factors on the brain}

A summary of the suggested mechanisms linking these risk factors to dementia are shown in figure 5. Vascular damage to the brain not only increases risk of microvascular and macrovascular lesions but also of atrophy and neurodegeneration. Oxidative stress and inflammation is associated with deposition of amyloid-beta (70). Diabetes and metabolic syndrome are associated with atherosclerosis and brain infarcts, and glucose-mediated toxicity causes microvascular abnormalities and neurodegeneration (71). Evidence of impaired insulin receptor activation in $A D(72)$ has led to suggestions that it may represent 'an insulin resistant brain state' (73). Exercising more in midlife is associated with a reduced risk of dementia (74). Exercise is postulated to have a neuroprotective effect, potentially through promoting release of Brain Derived Neurotrophic Factor (75) (76), reducing cortisol and reducing vascular risk. Exercise alone, as discussed below, does not appear to improve cognition in healthy older adults (77).

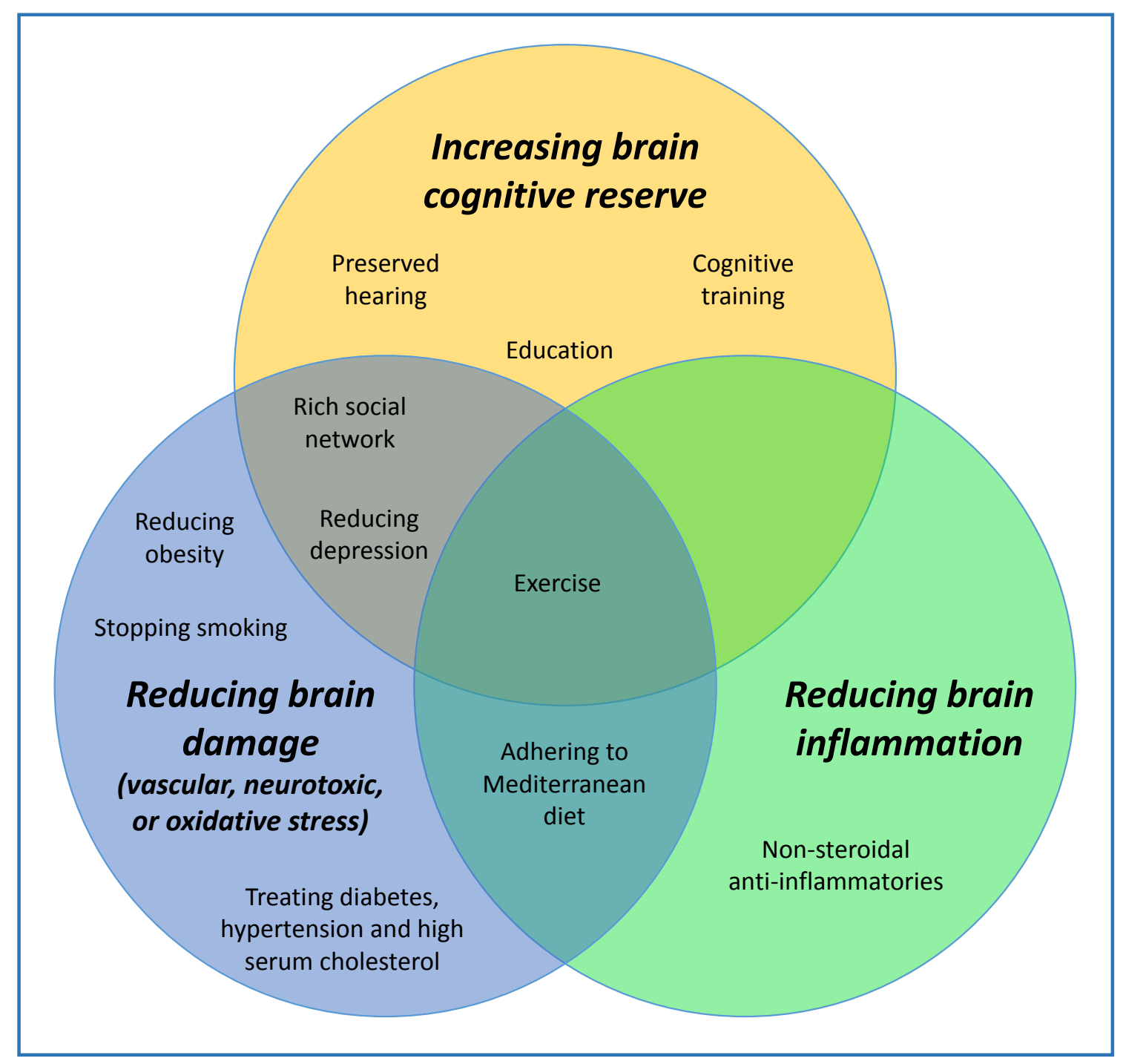

Figure 5. Potential brain mechanisms for preventative strategies in dementia 


\section{Specific risk factors and mechanisms}

We discuss the specific risk factors and their effect below and they are summarised in figure 5 .

\section{Education}

Less formal schooling is associated with a relative risk of dementia of 1.59 (95\% Cl [1.35-1.86]) and the high PAF is because of the large worldwide estimated prevalence of $40 \%$. Less time in education, which we defined as lack of secondary school education, has the second highest PAF in our model. Low educational level is thought to result in vulnerability to cognitive decline due to lack of cognitive reserve (56) which enables people to maintain function despite brain pathology (78). We do not yet know whether education post-secondary school is additionally protective.

\section{Hearing}

Recognition of hearing loss as a risk factor for dementia is relatively new and it has not been included in previous calculations of PAF, nor has it been a priority in the management of those at risk of cognitive impairment. Cohort studies that have investigated hearing have usually found that even mild levels of hearing loss increase the long-term risk of cognitive decline and dementia in those who are cognitively-intact but hearing-impaired at baseline $(63 ; 65 ; 79-87)$. However, while there are 11 positive studies, two found the increased risk was no longer significant in adjusted analyses $(88 ; 89)$.

The risk of hearing loss for dementia in the meta-analysis of three studies which we performed for this paper (figure 3, pooled relative risk $=1.94 ; 95 \% \mathrm{Cl}[1.38-2.73]$ ) is not only higher than the risk from other individual risk factors; but it is also pertinent to many people as it is highly prevalent, occurring in $32 \%$ of those aged $>55(90)$. Its high relative risk and prevalence explains the high PAF. We have used the prevalence of hearing loss in those over 55 to calculate PAF as this was the youngest mean age in which presence of hearing loss was shown to increase dementia risk (65). Hearing loss is therefore grouped with the midlife risk factors but evidence suggests it continues to increase dementia risk in later life.

The mechanism underlying peripheral hearing loss associated cognitive decline is not yet clear, nor is it established whether correction, such as hearing aids, can prevent or delay dementia's onset. Older age and microvascular pathology increase the risk of both dementia and peripheral hearing loss, and may therefore confound the association. Hearing loss may either add to the cognitive load of a vulnerable brain leading to changes in the brain (91), or lead to social disengagement or depression $(92 ; 93)$ and accelerated atrophy (94), all of which could contribute to accelerated cognitive decline (95).

While impaired hearing may detrimentally influence performance on formal cognitive assessments, those with impaired baseline hearing had normal baseline cognition so this cannot, however, account for the findings.

We lack experimental evidence on whether some of these negative effects may be alleviated by hearing aid use. Any intervention would require greater complexity than merely suggesting to people that they use a hearing aid. This is because only a minority of people with hearing loss are either diagnosed or treated (96), and when prescribed, many people do not use hearing aids (97).

Central hearing loss is distinct from peripheral hearing loss. It is a difficulty in understanding speech in noise, that is not explained by cochlear (peripheral) hearing impairment, and does not improve 
with peripheral amplification (such as hearing aids) (98). It is unlikely to be a modifiable risk factor and could be a prodromal symptom of Alzheimer's disease causing impaired speech perception, especially in the presence of competing sounds (99). This is consistent with the fact that central auditory areas are affected by Alzheimer's disease pathology (100). It is very unlikely that central hearing impairment would account for the association between peripheral hearing loss and dementia identified in studies, because the central hearing loss that is followed by Alzheimer's disease is rare, at $2 \%$ of the older population (99) while the prevalence of peripheral hearing loss in the studies included in the meta-analysis in a similar middle aged and older population is much larger (28\%, $43 \%$ and $58 \%$, depending on the specific study). Milder levels of central hearing loss might be more prevalent but they have not been linked to increased risk of dementia (101).

A recent small pilot intervention, Hearing Equality through Accessible Research \& Solutions (HEARS), used visual materials and training for the participant and a family member, to increase listening devices usage in cognitively healthy older adults and indicated that it may be possible to increase their use(102).

\section{Exercise and physical activity}

Older adults who exercise are more likely to maintain cognition, but like cognitive interventions, there is no current evidence that this prevents cognitive decline or dementia. One meta-analysis of 15 prospective cohort studies following 33,816 individuals without dementia for 1-12 years, found that physical activity had a significant protective effect against cognitive decline, with high levels of exercise being the most protective: hazard ratio 0.62 (95\% Cl 0.54-0.70) (103). Another metaanalysis included 16 studies with 163,797 participants without dementia and found that the relative risk of dementia in the highest physical activity groups compared to the lowest was $0.72(95 \% \mathrm{Cl}$ $0.60-0.86)$ and the relative risk of $A D$ was $0.55(95 \% \mathrm{Cl} 0.36,0.84)(104)$. Physical exercise leads to benefits in older people without dementia, including improving balance and reducing falls (105), improving mood (106), reducing mortality and improving function (107).

\section{Diabetes, hypertension and obesity}

Hypertension had the highest PAF amongst this group of risk factors, linked by their status as vascular risk factors, but they all had PAFs below $5 \%$ (108). Obesity is linked with pre-diabetes and metabolic syndrome which is characterised by insulin resistance and high levels of peripheral insulin. This is thought to cause a decrease in brain insulin production which can impair amyloid clearance (109). Increasing inflammation and high blood glucose concentrations may also be mechanisms by which diabetes impairs cognition (110).

\section{Smoking}

Smoking had the third highest PAF, in keeping with previous analyses (32). The association with cognitive impairment may be due to the link between smoking and cardiovascular pathology but cigarette smoke also contains neurotoxins which heighten the risk (111). Again, its high prevalence contributes to increasing the PAF and population level. Interventions are currently being used to reduce cigarette smoking and it has and is declining in most countries, although in 2015 it appeared to be increasing in the Eastern Mediterranean and Africa (112).

\section{Depression}

Depressive symptoms can be a part of the clinical presentation of dementia, which has led to debate as to the direction of causation; whether depression is a prodromal symptom or an independent risk 
factor for dementia. Cohort studies with longer follow-up times show a link between number of depressive episodes and risk of dementia, which strengthens the assertion that depression is a risk factor for dementia (113). The mechanism is likely to be multifactorial, as depression is linked to cerebrovascular pathology, and affects stress hormones, neuronal growth factors and hippocampal volume (114). In the past three decades there has been increased antidepressant prescription and this is hypothesised to impact on dementia incidence as animal data suggests that some antidepressants, including citalopram, decrease amyloid production (115-117).

\section{Social contact}

The PAF for social contact was similar to that of hypertension or physical inactivity. As with depression, social isolation may be a prodrome or a part of the dementia syndrome. However, there is a growing body of evidence that social isolation is a risk factor for dementia and it increases the risk of hypertension (118), coronary heart disease (119) and depression (120). Social isolation may also result in cognitive inactivity, linked to faster cognitive decline and low mood (60). All of these are risk factors for dementia themselves. This highlights the importance of considering the social engagement of older people and not only their physical and mental health.

\section{Lifestyle factors- Mediterranean diet and alcohol use}

Those who adhere to the Mediterranean diet (low meat and dairy, high fruit, vegetables and fish) have fewer vascular risk factors, and reduced plasma glucose and serum insulin levels, insulin resistance and markers of oxidative stress and inflammation (121). Not smoking, exercising regularly, eating fruit and vegetables daily and drinking only a moderate amount of alcohol increase life expectancy and health in ageing (122) so there is growing interest in these factors' impact on cognition. We do not have data to include dietary factors and alcohol in our calculations but they may be important.

\section{Head injury}

Most head injuries are mild and the commonest head injury is a non-repetitive traumatic brain injury (TBI). The largest study of TBI found that $12 \%$ of 7,130 participants in a 20 year longitudinal cohort study had a history of TBI (defined as $>1$ hour loss of consciousness). This was neither associated with a greater risk of development of dementia nor $A D$, nor increased plaques and tangles in the 1,589 participants who had an autopsy (123). TBI was associated with the development of Parkinson's disease and Lewy body pathology.

An earlier meta-analysis of seven studies, following people up at least one year after TBI, found it was not associated with increased risk of all cause dementia, but there was increased risk of AD (OR $1.40,95 \% \mathrm{Cl}$ [1.02-1.90]) (124), and there is some evidence that this effect is modified by sex; that the risk of dementia following TBI is greater for men $(125 ; 126)$. The meta-analysis also found there was no difference in risk between single and repetitive TBI. It concluded there were limitations in and heterogeneity of studies.

It is unclear what short-term brain pathology is typically caused by a single blast related head injury (127). Repetitive mild head injury in athletes or war is associated with chronic traumatic encephalopathy, a progressive tauopathy, which can eventually manifest as dementia (128). The Institute of Medicine has concluded that moderate or severe TBI, such as in war, is a risk factor for $A D$ (129) but overall the evidence appears to be that non-repetitive TBI does not predispose to all cause dementia. 


\section{Other factors}

Visual impairment and sleep disorders have received some attention for their role in the development of cognitive impairment (57). Sleep might promote repair of damage caused by other factors but given the lack of systematic reviews or enough consistent, high quality evidence we have not been able to include it in our calculations of PAF. Bilingualism may also be protective, as a cognitive activity (130) but results from prospective cohort studies regarding its protection against cognitive decline have been mixed $(131 ; 132)$. One longitudinal study has found that living near major roads increases the chance of having a recorded diagnosis of dementia (133).

\section{Limitations of the data}

\section{Causality in longitudinal studies}

The PAF model assumes a causal association between a risk factor and dementia, and a causative link is required for interventions to lead to actual reductions in the incidence of dementia. With regard to causality, the most convincing evidence of causality would be randomised controlled trials in humans. This is not possible for many proposed dementia risk factors such as education; but we know that falling age-specific incidence is associated with more education (25). In the absence of this experimental human evidence, causality criteria have been proposed (134).

The emergent risk factors in our publication, including hearing loss and low social engagement, meet these criteria, suggesting plausible causal relationships. For hearing loss, for example - (1) Strength of association: Our meta-analysis showed effect size of 1.94 (95\% Cl [1.38-2.73]); (2) Consistency: the three high quality cohort studies identified in our meta-analysis reported a statistically significant association between peripheral hearing loss and dementia, with overlapping $95 \%$ confidence intervals; (3) Temporality: the studies measured hearing loss, then followed non-demented people for at least 9 years, identifying incident dementia cases during this follow-up; (4) Biological gradient: There is a dose-response whereby mild, moderate or severe hearing loss lead to increased relative risk of dementia of 1.89, 3.00 and 4.94, respectively(135); (5) Plausibility: In animal models, hearing loss precedes changes in brain structures (136), volume (137) and networks(138). Improving hearing (and social and exercise interventions) might improve cognition by environmental enrichment, associated with reduced amyloid deposition in mice models (139). There are likely additional humanspecific mechanistic pathways because of the importance of language relative to other species; language is a key element of the co-evolution of larger brain size, social interaction and larger-scale group cooperation in humans (140). Hearing loss in humans may therefore result in uniquely interrelated and detrimental social, cognitive and brain effects.

\section{Modifiability of the risk factors}

PAF reflects the proportional reduction of incident dementia cases that current evidence suggests would not occur if risk factors were eliminated. This figure should be interpreted with caution as it is not feasible to completely eliminate any of these risk factors and some risk factors can also be part of the dementia syndrome. However, our understanding of what we could and should target provides an opportunity to consider better management or preventive strategies to reduce the burden of risk. 


\section{Differences in PAF estimates}

Our assessment of the effect of potentially modifiable risk factors is higher than previous estimates reported but we have incorporated two additional risk factors, one of which, hearing loss, is extremely common in middle and later life, so would be expected to have a high PAF. We have used data to calculate communality from the Health Survey for England from 2014, whereas previous estimates used data from 2006. We have made our estimates as conservative as possible by calculating communalities for adults $>65$ years of age, as this is the age-group most vulnerable to dementia, and correlation between risk factors is likely to be more relevant in this age-group rather than in all adults.

\section{When in the life course is a risk factor important?}

While we have presented the current evidence about specific times when a risk factors has been shown to be important during the life course, they may be relevant at other times. It may be that ongoing education continues to increase cognitive reserve. Similarly, diabetes, hypertension, depression, being sedentary and smoking are probably important in middle age, and later life, and hearing loss may be a risk in late as well as mid-life.

\section{Other risk factors not in our model}

We have not incorporated other factors, such as diet, alcohol, traffic or sleep which may be relevant. It is therefore possible that the potentially preventable fraction of dementia is underestimated in our figures.

\section{Reverse causality}

The direction of causality is sometimes unclear and may sometimes be bidirectional. For example, reduced socialisation or increased depressive symptoms may be caused by, and cause, cognitive decline and thus our figures may be an overestimate. When considering some risk factors which occur not long before the onset of impairment, it is difficult to be sure of direction of causation; e.g., whether depression increases the risk of dementia or dementia increases the risk of depression or if the relationship is bidirectional.

\section{Communality of risk factors}

Our communality calculations take into account shared mechanisms of reversible risk factors but it is also possible that genes may predispose to both dementia and hypertension, depression or hearing loss.

\section{Global estimates of prevalence}

The prevalence of risks we have used are from the largest populations we could find, but these are not always global and will differ in different parts of the world with varying cultures and incomes. We have also used conservative estimates of prevalence for social isolation or hearing loss aged over 55 , to produce as accurate an estimate of PAF as possible.

\section{Data quality}


Finally, there are differences in the quantity of data so that the estimates for hearing loss are less stable than those for hypertension, smoking or diabetes, for example, since there are fewer studies that that have been used to contribute to the estimates presented.

\section{Importance of PAF findings}

The general principle is that there is an important proportion of modifiable risk factors in dementia, whether we assume the true PAF to be lower or higher than our estimate. This could translate into a large impact on the global burden of dementia which would then have huge implications for social and healthcare costs.

While public health interventions will not delay, prevent or cure all potentially modifiable dementia, the management of metabolic, mental health, hearing and cerebrovascular risk factors may push back the onset of many cases for some years. Dementia prevalence would be halved if its onset were delayed by five years (141). Estimates are that worldwide dementia prevalence would be reduced by over a million cases, with a $10 \%$ reduction in the prevalence of the seven principal health and lifestyle factors; or an intervention that delayed dementia by a year, might decrease the number of people living with dementia globally by 9 million in 2050 (32;59). While we might not expect risk factor modification to have this magnitude of impact in reality, any reduction in dementia risk would be a significant achievement.

\section{Interventions to prevent dementia}

\section{Single agent trials to prevent dementia}

The existence of potentially modifiable risk factors does not mean that all dementia is preventable or make it more treatable once established. There have been intervention studies building on the evidence of modifiable dementia risk factors to reduce dementia incidence, testing the effects of physical activity, cognitive training, and medication including antihypertensives (142;143). The low dementia incidence rates meaning that trials have to be large to and long to show a reduction in dementia cases and with the multiple risk factors contributing to dementia may explain why most prevention trials have been inconclusive (144), leading to the development of multi-modal preventative strategies.

\section{Antihypertensives}

While most interventions trials have been ineffective, the exception is antihypertensives. A trial in non-demented but hypertensive(defined as $160-200 /<110 \mathrm{mmHg}$ ) people aged $>80$ years, of the antihypertensive indapamide, with the option of perindopril, was stopped early because a reduction of stroke and mortality in the treatment group meant it was unethical to continue placebo (145). Therefore the trial did not fulfil the power calculation and the $95 \%$ confidence intervals overlapped between treatment and placebo groups (hazard ratio [HR] 0.86, 95\% Cl [0.67-1.09]). However, when these data were combined in a meta-analysis with other placebo-controlled trials of antihypertensive treatment the combined risk ratio for dementia favoured treatment (HR 0.87, 95\% $\mathrm{Cl}[0.76-1.00])$ (145). Similarly, a meta-analysis showed there was a reduction in cognitive decline in the treatment groups (weighted mean difference $=0.42 ; 95 \% \mathrm{Cl}[0.30-0.53]$ ) (146). This was consistent with an RCT, aiming to reduce systolic blood pressure to $<150 \mathrm{~mm} \mathrm{Hg}$ in people aged $>60$ without dementia using nitrendipine (10-40 mg/day) with the possible addition of enalapril (5-20 $\mathrm{mg} /$ day) or hydrochlorothiazide (12.5-25 mg/day) which reduced dementia's incidence rate (147). In 
the pre-Diva trial discussed below, the treatment of hypertension also appeared to be important (148). The benefits of strictly managing hypertension must be balanced with risks and target blood pressure for people aged $>80$ years should be below $150 / 90 \mathrm{mmHg}(149)$.

\section{Non-steroidal anti-inflammatory drugs, hypoglycaemic agents, vitamins, hormone replacement, ginkgo, statins}

By contrast, trials of non-steroidal anti-inflammatory drugs (NSAID) (150), a 24 week RCT of an oral hypoglycaemic drug, rosiglitazone (151), oestrogen hormone replacement therapy, statins (152), vitamins and ginkgo biloba extract have all been negative (3).

There is good evidence that statins do not prevent (or increase) cognitive impairment or dementia from two negative trials with 26,340 participants aged 40 to 82 years of whom 11,610 were aged 70 or older with risk factors for vascular disease (152).

While several meta-analyses have shown HRT to have a $29-44 \%$ protective effect against dementia, a US study observed negative effects (153). The latter study was, however, on women many years after the menopause and at high cardiovascular risk. More recent reviews of both observational and intervention studies conclude there are overall neither harmful nor beneficial effects in relation to dementia, with negative effects being more likely in women in poor health, especially those with cardiovascular disease and diabetes (154). HRT cannot presently be recommended to prevent dementia; however, it is possible that there may be beneficial effects for a sub-group of healthy women receiving treatment in the peri-menopausal period. Furthermore, most research was in women taking orally administered conjugated equine oestrogens and progesterone, and the longterm effects of more recently developed molecules and transdermal administration are unknown.

\section{Mediterranean Diet}

Four hundred and forty seven healthy participants, with a mean age of 67 years, at high cardiovascular risk but with no cardiovascular disease or significant cognitive impairment were randomly assigned to one of three dietary groups (155). These were a Mediterranean diet supplemented with extra virgin olive oil (1 L/week), a Mediterranean diet supplemented with mixed nuts $(30 \mathrm{~g} / \mathrm{d})$, or a control diet (advice to reduce dietary fat) and adherence to the supplements was measured by urine testing. In the primary analysis of composite cognitive change over 4 years, those in intervention groups did better than the control group. Secondary analysis of the numbers developing $\mathrm{MCl}$ found no significant difference, and no participants developed dementia, suggesting that this intervention may have effect on cognitive aging but not the dementia syndrome. Participants who withdrew had worse baseline cognition and more APOE $\varepsilon 4$ genotypes than completers, thus being more likely to be cognitively impaired at follow-up, and there were more dropouts in the control group, which suggests that the intervention's benefits may have been underestimated.

\section{Cognitive interventions}

Initial evidence that engaging in cognitively stimulating activities may benefit cognition and reduce dementia risk came from epidemiological studies. One study assessed the frequency of participation in seven common mentally-stimulating activities at baseline and followed up 801 older adults without dementia for 4.5 years (108). A 1-point increase in the cognitive activity score was 
associated with a 33\% reduction in the risk of AD. A meta-analysis of 29,279 individuals from 22 longitudinal cohort studies with a median follow up of 7.1 years calculated a summary odds ratio (OR) of incident dementia for high vs low engagement in mentally-stimulating activities of 0.5 (95\% $\mathrm{Cl}$ [0.42-0.61]), after controlling for other dementia predictors such as age, sex, general health, cerebrovascular disease, education, occupation and baseline cognition (56). This suggests that cognitive reserve is not a static property, but may be amenable to manipulation by cognitive interventions in later life.

There is some evidence of generalised cognitive improvements from either single domain or reasoning training in healthy older people but not currently of prevention of cognitive decline or dementia. When 2,802 healthy older people were randomised to receive ten group sessions focusing on attention, memory or reasoning, there were improvements within the trained domains (156), with functional benefits at 10 year follow up (157). A recent online study compared reasoning training with general cognitive training and an active control in 6,742 participants, of whom 2,912 were aged $>60$ years. Although there was significant dropout over the six month study, reasoning training demonstrated generalised benefits in both trained and untrained measures of executive function ( $d=0.42$ ), on activities of daily living (effect size $d=0.15$ ) and verbal learning $(d=0.18$ ) (158). The combination of cognitive training with other lifestyle interventions in the FINGER trial is described below (159). The commercial brain training tools that are widely promoted often have efficacy claims that are not substantiated by real evidence that they can prevent cognitive decline.

\section{Exercise and physical activity interventions}

RCTs of exercise interventions for cognition in healthy older adults have been less successful than might have been expected from the longitudinal cohort studies. Recent meta-analyses have either reported no overall evidence that exercise improves cognition in healthy older adults (77), or that benefits are limited to specific cognitive domains. A recent meta-analysis reviewed 25 studies of aerobic exercise, resistance training or Tai Chi (160). Fifteen of these reported significant improvements for exercise vs controls on measures of executive function, memory or composite measures of cognition. However, the only significant results from the meta-analysis were for resistance training improving reasoning vs controls (two studies, mean difference $=3.16(95 \% \mathrm{Cl}$ [1.07-5.24] 135 participants) and Tai Chi improving processing speed and attention vs no exercise control (two studies, 156 participants). Conversely, a meta-analysis of 29 studies of aerobic exercise in healthy adults, including three studies of participants with $\mathrm{MCl}$, found overall exercise related improvements in people with MCl's memory (Hedges' $g=0.237, p=0.05$ ) (161). A recent RCT of 100 adults with $\mathrm{MCl}$, randomised to resistance training or cognitive training, reported that resistance training significantly improved the primary cognitive outcome, ADAS-Cog (effect size $=-0.33(95 \% \mathrm{Cl}$ [ - 0.73-0.06])) at 6 months and executive function at 18 months (162). The potential mechanisms for physical exercise to improve cognition or prevent dementia are indirect effects on other modifiable risk factors such as obesity, insulin resistance, hypertension, hypercholesterolaemia and general cardiovascular fitness; and direct neurological effects such as increased neurogenesis, cerebral blood flow, and levels of Brain Derived Neurotrophic Factor(76;163;164). It is suggested that some interindividual variability in response contributing to the conflicting RCT findings may be related to individual differences in exercise-related neuroplasticity (165). It may also be that protective effects in long-term studies accumulate over years rather than over a short time and that people who exercise are different in several ways to those who do not. One RCT of 40 minutes walking three 
times weekly for a year (versus stretching and conditioning) showed exercise training increased hippocampal size and improved memory in healthy adults aged 55-80 (166). Overall there is not sufficient scientific evidence that physical activity reduces dementia risk (167).

\section{Social engagement}

Longitudinal studies suggest that social interaction may prevent or delay dementia but there is a lack of intervention study evidence that social activity prevents cognitive decline or dementia. People who live alone, have never married, are divorced or widowed have an increased risk of all cause dementia (168). A recent meta-analysis of social activity found that incident dementia risk was elevated for people with more limited social activity participation (RR: $1.41,95 \% \mathrm{Cl}$ [1.13-1.75]) and less frequent social contact (RR: $1.57,95 \% \mathrm{Cl}$ [1.32-1.85]) (60) but not those who had low satisfaction with social contact. The relatively short follow-up period in some studies precludes strong conclusions about the direction of causation.

People with dementia may be less motivated to engage socially or find more difficulties in organising activities, be embarrassed by their difficulties or worried they may be unable to manage previous activities or may get lost. Social norms and low tolerance for cognitive decline of others can result in increasing isolation of many people with dementia. At early stages of cognitive decline, people report feeling lonelier than people with intact cognition (169). While many family members may increase contact as the person with dementia requires more support, visits by family members tend to decrease as the dementia becomes more severe, as relatives may find it distressing or be unsure that their relative gains from their visits. People with more severe dementia may move homes for support further from their previous social support network.

There is little knowledge about the effect of social activity interventions on cognition. One pilot RCT for older adults with social activity as an intervention component found adults with impaired executive function showed significant improvements (170). Another pilot RCT compared cognitive training, health promotion course, and a book-club as interventions for people with subjective memory problems but not dementia and found no between group difference (171)

\section{Studies using combination strategies to prevent dementia}

\section{The FINGER study}

The Finnish Geriatric Intervention Study to Prevent Cognitive Impairment and Disability (FINGER) $(159 ; 172)$ provided four intensive lifestyle-based strategies (diet, exercise, cognitive training and vascular management) to over 600 people, aged over 60 , who were at high risk of dementia according to their age, sex, education, systolic blood pressure, total cholesterol and physical activity (173). It compared cognition in the intervention group to controls who received general health advice. This highly intensive intervention consisted of about 200 meetings (300 hours) with health professionals and trainers over two years.

Participants in the intervention group showed a mean improvement versus the control group in a composite measure of cognition (Cohen's $d=0.13$ ) on executive function and processing speed, but not memory. Despite the intervention's intensity, the effect was small, although this demonstrates potential for lifestyle modification to improve cognition function in people at risk of dementia. Pragmatic multi-modal models for dementia prevention should be tested in other populations and 
settings (159). Earlier intervention and longer follow-up will determine whether these approaches reduce dementia risk.

\section{The pre-DIVA study}

The Prevention of Dementia by Intensive Vascular Care (preDIVA) study in the Netherlands (174) also aimed to reduce vascular risk factors to prevent dementia in a six-year multi-domain, nurseadministered vascular care intervention open-label, cluster-randomised controlled trial (RCT) with a total of 3,526 participants from general practice. Smoking habits, diet, physical activity, weight, and blood pressure were monitored and individually-tailored lifestyle advice according to protocol was provided, supported by motivational interviewing. Blood glucose and lipid concentrations were assessed every two years in both groups and when indicated otherwise. If indicated, medication was given for hypertension, diabetes or dyslipidaemia. There was no significant difference in dementia incidence between the intervention and usual care group over 6.7 years (hazard ratio [HR] 0.92, 95\% $\mathrm{Cl}[0 \cdot 71-1 \cdot 19])$ (148). The authors thought the negative findings may have been related to the relative lack of cardiovascular risk factors in the study population, reducing the possibility of risk reduction. An accompanying editorial noted that, in the intervention group, $10 \%$ more of the participants who were not using antihypertensives at baseline were subsequently treated, and in those participants, there was reduced risk of dementia (22 [4\%] of 512 intervention developed dementia vs 35 [7\%] of 471 control; HR 0.54, 95\% Cl [0.32-0.92]) (175). These outcomes illustrate the importance of targeted interventions and of a clear model linking risk factors to dementia.

\section{Other multidomain studies}

The Multidomain Alzheimer Preventive Trial in France (MAPT; NCT00672685) (176) with multidomain lifestyle interventions and the Healthy Aging Through Internet Counselling in the Elderly (HATICE) (177) project have similar aims, the latter using a less costly e-health intervention, but the results are not yet available.

\section{Dementia intervention: what, when, for how long and for whom?}

Such programmes are not yet ready for implementation as large-scale public health interventions. This is related to the desire for conclusive RCTs to confirm efficacy; the cost and intensity of interventions needed to change behavior; and doubts as to the underlying aetiology of dementia. There are, however, numerous examples where public health interventions have reduced disease incidence before the disease process has been understood e.g. hand-washing reducing puerperal fever, clean water eliminating cholera and condoms reducing HIV transmission. Risk reducing strategies in many countries in cardiovascular and metabolic health, cigarette smoking, depression, social and physical activity, and hearing may be implemented and might account for the decreased incidence of dementia in more recent cohorts. 
Although dementia is diagnosed in late life, pathology develops years earlier (178). Increasing evidence from epidemiological, clinical, imaging and biomarker studies suggests that dementia, especially $A D$, may be a clinically silent disorder starting in mid-life, whose terminal phase is characterised by dementia $(179 ; 180)$. A fundamental question is, therefore, when in the lifespan should dementia prevention programmes be implemented and for how long? Studies to date appear to show that providing modestly enhanced care to non-targeted patients already receiving medical care does not reduce dementia (175).

\section{Key points and recommendations}

Preventing or delaying dementia onset is a public health priority with potential to reduce not only the disability of individuals but also associated societal and economic burden. In many countries dementia is already being delayed for years. Thus while trials, which by their nature are relatively short and include a smaller number of people, are disappointing, results from risk factor modification for whole populations or high risk populations have been more hopeful. Dementia may constitute the terminal stage of disease processes beginning decades earlier, and lifestyle changes targeting these processes may sometimes prevent or delay dementia onset. There is good evidence that treating hypertension reduces dementia incidence and preliminary evidence that modifying several risk factors has a beneficial effect on cognition. The interventions most likely to be beneficial (increasing education in early life, increasing physical activity and social engagement, reducing smoking, treating hypertension, diabetes and hearing impairment) are safe and confer other health benefits.

\section{Early detection: Preclinical AD}

Preclinical AD occurs when there are early Alzheimer's pathogenic changes but no memory impairment (181). These pathogenic changes in AD include extra-cellular deposition of, beta amyloid also known as Abeta protein, from cleaved amyloid precursor protein which is the main component of plaques; and intracellular accumulation of Tau protein which is the main constituent of tangles.

The main purpose of preclinical detection of $A D$ is to identify individuals at high risk of progression to dementia due to $A D$, so that they can have the opportunity to participate in treatment trials to delay or prevent cognitive decline. They can also be informed and make changes in their lifestyle which may delay dementia's onset. Some people may also find prognostic information to be useful, as it allows them to make plans and lifestyle changes for a possible future dementia.

Many or even most of those found to be at risk of dementia will die in good cognitive health, at a merely theoretical risk of developing dementia, and thus it is important that risk information, e.g., amyloid scans results, is presented cautiously as it has the potential to cause harm without compensatory benefit. The potential of early detection will be realised if effective AD modifying treatments for these stages are developed, in which case detection would be essential in order to determine to whom such treatments should be offered and services would have to change and expand to accommodate this. The ethical implications of pre-dementia biomarker testing are profound but have yet to be worked out in any detail (182).

Preclinical $A D$ is also known as "asymptomatic at-risk state for $A D$ " as it is uncertain what the predictive value is of this pathology. Those with rare familial $A D$ are sometimes termed as having "presymptomatic $A D$ " and are expected to develop $A D(183)$. AD has an insidious onset and most 
people pass through a preclinical asymptomatic phase when cerebral A $\beta 42$ amyloid and other abnormal proteins are accumulating in the brain, followed by $\mathrm{MCl}$, and ultimately progress to dementia (184). Abnormal biomarkers are common, with 10 to $30 \%$ of cognitively normal older people, depending on age, having significant brain amyloid deposits in PET scanning; these increase with age; and are more likely to be high in those with the APOE $\varepsilon 4$ allele (185). These follow-ups have been in highly selected populations and we do not know their predictive value in more general populations of older people. Most cognitively normal older people, with significant amyloid depositions detected in a scan, do not decline clinically over the following 18-36 months (186). However, amyloid positivity on scan was the most significant predictor of progression to dementia from $\mathrm{MCl}$ in one study, with 59\% progressing to dementia within 3 years (187). Similarly, 3-year conversion from $\mathrm{MCl}$ to $A D$ was predicted by low baseline CSF (equivalent to high brain) amyloid- $\beta$ levels (188). A small, three year, longitudinal study of 32 cognitively normal, amyloid positive older adults, found $25 \%$ had developed $\mathrm{MCl}$ or dementia due to $\mathrm{AD}$ over three years, while only one of 73 of those with a negative amyloid scan developed $\mathrm{MCl}$ (189). Overall, although amyloid deposition is a risk for the development of $A D(190)$ its precise predictive value is still unknown (185).

Numerous pharmacological compounds have been developed over the past few decades to combat dementia $(3 ; 191)$. The results of trials have all been negative and consideration is now being given to drug development for earlier disease stages, so-called preclinical $A D$, characterised by biomarkers or the pathology of $A D$ without signs or symptoms. For example EPAD (the European Prevention of Alzheimer's Disease program), a Horizon 2020/Innovative Medicines Initiative in collaboration with the EFPIA (European Federation of Pharmaceutical Industries and Associations) is designed to address this question by developing a platform able to deliver large pre-clinical proof of concept trials for both existing and newly developed compounds (192). A central problem, however, for both prevention and disease modifying interventions is outcome measures. If treatment is to be given to cognitively- and functionally-intact persons in the decades prior to dementia onset, then the outcome measures could be biomarkers or time to dementia diagnosis. The latter would take large populations and many years of follow-up. Any evaluation should include side effects, as these may limit long-term treatment. Further information on cognitive, imaging and biomarker is needed to establish what should be measured and to determine treatment effect size.

Cohorts of healthy older people and those at risk, such as the PREVENT study (193), Alzheimer's Disease neuroimaging initiative (ADNI) (194) and Dominantly-inherited Alzheimer's network (DIAN) (195), are currently being assembled for these purposes. There are now several clinical trials aimed at prevention in people who are cognitively well but at higher risk of Alzheimer's disease by virtue of genetics or biomarkers (196).

\section{Key points and recommendations}

Depending on their age, $10-30 \%$ of cognitively normal older individuals have abnormal brain amyloid or $A B$ and tau levels in CSF. Only a minority of those adults will progress to $\mathrm{MCl}$ or dementia due to $A D$ over three years. There are potential ethical concerns about identifying a population at risk of dementia, many of whom may not develop dementia in their lifetime. Therefore, at present the main purpose of biomarkers is to identify and characterise higher risk individuals to take part in trials. 


\section{Mild Cognitive Impairment}

Mild cognitive impairment ( $\mathrm{MCl}$ ) is also sometimes called Cognitive Impairment No Dementia (CIND) $(197 ; 198)$. It has been defined as an objective cognitive impairment, reported by a patient or relative, in a person with essentially normal functional activities, who does not have dementia (199). It can broadly be considered as an intermediate state between normal aging and early dementia, which sometimes reverts to normal cognition. $\mathrm{MCl}$ is probably best conceptualised as a probability state which can be used to delineate a population at higher risk of dementia, with cognitive decline not meeting diagnostic criteria for dementia. People with $\mathrm{MCl}$ are clinically and neuropathologically heterogeneous (197). It affects many more people than dementia does, and estimates of prevalence vary from $4-19 \%$ of people aged $\geq 65$, depending on the definition used and how it is interpreted $(198 ; 200 ; 201)$. Functional loss secondary to cognitive impairment has previously been the entry point of persons with neurodegenerative disorders into the health and social care system but many people now present with $\mathrm{MCl}$. Around $39 \%$ of those diagnosed with $\mathrm{MCl}$ in specialist settings and $22 \%$ in population studies, develop dementia over the subsequent three years(202), compared to $3 \%$ of the non- $\mathrm{MCl}$ population of the same age (203). $\mathrm{MCl}$ can be divided into amnestic $\mathrm{MCl}$ (aMCl) defined as those with a particular impairment of episodic memory (204) often thought to be likely to develop into $\mathrm{AD}$; and non-aMCl.

\section{Prodromal AD}

People with $\mathrm{aMCl}$ and a positive CSF $\mathrm{A} B$ and tau biomarker, or positive $\mathrm{A} B$ PET scan, have been termed as having prodromal $A D(181 ; 183)$ or $\mathrm{MCl}$ due to $A D(205)$ an advance over the heterogeneous term $\mathrm{MCl}$. This subgroup is more likely to progress to Alzheimer's Dementia (199). In other subgroups, $\mathrm{MCl}$ may be caused by vascular pathology, or herald other types of dementia.

Development of future $\mathrm{MCl}$ interventions should recognise this heterogeneity, or direct specific interventions at homogenous sub-groups, for example, those likely to have prodromal AD. However, if disorders such as $A D$ can be diagnosed in the preclinical or prodromal period then treatment would ideally be given then.

\section{Risk factors for progression from $\mathrm{MCl}$ to dementia}

There is evidence from prospective studies, summarised in a systematic review, that diabetes, prediabetes, metabolic syndrome, lower serum folate levels, and the presence of neuropsychiatric symptoms increase the risk of progression from $\mathrm{MCl}$ to dementia, but less education does not (206). Mediterranean diet decreases the risk of conversion from amnestic $\mathrm{MCl}$ to $\mathrm{AD}(206)$. A slightly different view emerged from a large but unreplicated community cohort study where people were retrospectively classified as having $\mathrm{MCl}$ (207). It suggested that risk factors for progression to dementia differed between men and women; and interventions should focus principally on risk of stroke in men and depressive symptomatology and reducing anticholinergic medication in women (208).

The concept of mild behavioural impairment (209) is proposed to describe people at an increased risk of dementia due to the presence of late-life acquired neuropsychiatric symptoms such as apathy, affective symptoms, impulse control problems or social inappropriateness, which are viewed in this context as being prodromal dementia symptoms. A third to three-quarters of people with $\mathrm{MCl}$ 
have neuropsychiatric symptoms; most commonly depression, anxiety, apathy and irritability (210). Some of the symptoms may be a reaction to the experience of declining abilities. Neuropsychiatric symptoms may be indicators of people who are at higher risk of dementia as they predict conversion to dementia (206). Nonetheless, neuropsychiatric symptoms may be implicated in the aetiology of dementia, through neuroendocrine axis activation; or interact synergistically with a biological factor, such as genetic predisposition. Either of these putative relationships suggests treatment might have potential to delay dementia but it is unclear whether they are truly potentially modifiable risk factors rather than indicating individuals who are further along the path to a dementia syndrome.

\section{PAF for Modifiable Risk Factors in $\mathrm{MCl}$}

In order to highlight the potential for slowing progression of $\mathrm{MCl}$ to dementia, we have calculated the PAF using the formula above, for those modifiable risk factors shown in systematic reviews to affect the rate of progression. These are having diabetes, the presence of neuropsychiatric symptoms and not adhering to a Mediterranean-style diet. The individual risk factor PAFs represent the percentage of people who would theoretically not develop dementia from $\mathrm{MCl}$ if that risk factor could be completely eliminated. The direction of causality of neuropsychiatric symptoms discussed above does, however, remain.

We calculated communality for these risk factors using data on people aged $>65$ years from the Health Survey England using the methods described above. In the absence of data on Mediterranean diet, we used obesity as a proxy measure for not following a Mediterranean diet; and for neuropsychiatric symptoms, we used depression. We have also conservatively assumed these prevalences in people aged $\geq 65$ years are the same as in the population with $\mathrm{MCl}$. The principal component extracted using this method explained $45 \%$ of the total variance between the three risk factors. Using these methods, we calculated that $21.7 \%$ of dementia progression from $\mathrm{MCl}$ is potentially preventable by eliminating poor diet, diabetes and neuropsychiatric symptoms (assuming these are risk factors for, not symptoms of, or the result of dementia). Table 2 shows data on relative risks, prevalence and communalities and the PAF are These risks are ones for which we have data but that other factors, including hearing and social interaction, may be important in $\mathrm{MCl}$ but there is a lack of evidence at present.

\begin{tabular}{lccccc}
\hline \multicolumn{1}{c}{ Risk Factor } & $\begin{array}{c}\text { Relative risk } \\
\text { for dementia }\end{array}$ & $\begin{array}{c}\text { Prevalence } \\
(\%)\end{array}$ & $\begin{array}{c}\text { Communality } \\
(\%)\end{array}$ & $\begin{array}{c}\text { PAF } \\
\text { (\%) }\end{array}$ & $\begin{array}{c}\text { Weighted } \\
\text { PAF* (\%) }\end{array}$ \\
\hline Diabetes & 1.65 & 6.4 & 7.6 & 4.0 & 1.5 \\
\hline Neuropsychiatric symptoms & 2.52 & 29 & 61.1 & 30.6 & 11.5 \\
\hline Diet & 1.92 & 32.5 & 66.7 & 23.0 & 8.7 \\
\hline $\begin{array}{l}\text { Total adjusted for } \\
\text { communality }\end{array}$ & & & & & $\mathbf{2 1 . 7}$ \\
\hline
\end{tabular}

Table 2. Potentially modifiable risk factors for progression to dementia from $\mathrm{MCl}$; their relative risk, prevalence and population attributable fraction

Notes: *Weighted PAF is the relative contribution of each risk factor to the overall PAF when adjusted for communality

We used population prevalence of obesity and depression as proxies for diet and neuropsychiatric symptoms respectively. 


\section{Interventions to reduce or delay conversion of $\mathrm{MCl}$}

People with $\mathrm{MCl}$ have almost all been diagnosed after requesting a memory assessment, and are seeking to reduce their risk of dementia so have relatively high motivation to change. NICE recommends follow-up, so if dementia is diagnosed, planning can begin at an early stage, but no specific treatments (211). A recent $\mathrm{NIH}$ report recommended trials of interventions for dementia prevention encompassing multiple risk factors and targeting high risk individuals (172).

Multimodal interventions are likely to be needed to prevent progression to dementia in $\mathrm{MCl}$. This may involve approaches to decrease neuropathological damage (treating vascular risk factors, diabetes, diet, exercise) and treat neuropsychiatric symptoms, combined with those that maximise function (cognitive and social stimulation, treating neuropsychiatric symptoms). Understanding which components are useful and how to streamline and make these interventions cost-effective will be challenging.

\section{Cognitive interventions for $\mathrm{MCl}$}

A recent systematic review identified six studies of cognitive training in participants with $\mathrm{MCl}$. Four studies reported significant improvements on objective memory outcomes immediately following training, however only one out of three studies including general cognitive outcomes reported benefits (212). Similarly, global cognition did not improve with cognitive training in three small trials, in one of which it was a primary outcome, and there were no consistently significant findings on other secondary outcomes (213).

\section{Exercise interventions for $\mathrm{MCl}$}

There is mixed evidence that exercise can improve cognitive outcomes in $\mathrm{MCl}$. In a review of 14 studies, $92 \%$ of cognitive outcomes reported were non-significant, and only $42 \%$ of effect sizes were classified as potentially clinically relevant $(E S>0.20)$ (214). A systematic review found memory did not improve with exercise (213). In one very high-quality study, there was no effect of a year-long moderate aerobic exercise group compared with relaxation, balance and flexibility exercise active control, although post-hoc analysis showed some effect in individual domains in woman and different effect in men (215). The results of less high quality studies were mixed but did not indicate generalised cognitive improvement compared to control (213). Overall, there is no conclusive evidence for exercise in $\mathrm{MCl}$.

\section{Medication for $\mathrm{MCl}$}

A recent systematic review found no evidence that any drug interventions, cholinesterase inhibitors (ChEls) delay conversion to dementia in a general population with $\mathrm{MCl}$ (213). However, phase 2 studies of aducanumab in patients with prodromal or mild AD found it reduced amyloid in the brain in a dose-dependent fashion and phase 3 studies are now taking place (216).

\section{Cholinesterase inhibitors}

There was no reduction in incident $A D$ in four higher quality trials where this was the primary outcome - two evaluated galantamine, one donepezil, and one rivastigmine (213). Donepezil improved global cognition in one high quality trial where it was a primary outcome measure, and a second where it was a secondary outcome, but global cognition did not improve in the three other large, high quality trials of ChEls. Post-hoc analyses of RCT data indicate some benefit in specific 
populations characterised by the presence of biomarkers. There was less cerebral atrophy in people taking galantamine who have the ApoE $\varepsilon 4$ allele than those with other ApoE variants (217), and cognitive response to donepezil was higher in butylcholinesterase-K carriers than those with other genotype profiles (218). However, these post-hoc analyses should be treated with caution as no study has found a subtype difference when that was the primary hypothesis. Additionally, no studies have reported on functional effects or rate of progression to dementia.

\section{Non-steroidal anti-inflammatory drugs}

Trials have not shown non-steroidal anti-inflammatory drugs (NSAID) to be effective in $\mathrm{MCI}$ (213;219). One high quality study found that Rofecoxib, a cox-2 selective inhibitor increased incident cases of AD. A smaller study found triflusal (versus placebo) had no significant effect on cognition as a primary outcome measure, although it was associated with a reduced risk of the secondary outcome, conversion to AD. Several trials of NSAID have had to be stopped because of safety concerns. As any beneficial anti-inflammatory effect may be long-term, people with $\mathrm{MCl}$ may not be the appropriate treatment population (219).

\section{Statins}

We could not find any interventional trials of statins. One longitudinal observational study found statins did not affect cognitive decline in people with $\mathrm{MCl}(220)$.

\section{Vitamin B and E and folic acid}

Vitamin $E$ did not reduce incident dementia or impact on a range of secondary outcomes in one high quality study (221). Two placebo-controlled trials found that B vitamins (B12 and B6 plus folate) had no significant effect on immediate memory over 6 months (215) or global cognition (222).

\section{Ginkgo biloba}

On primary outcomes, $240 \mathrm{mg}$ daily Ginkgo biloba did not reduce incident dementia, AD or cognitive decline over 6 years in high-quality trials (223-225).

\section{Key points and recommendations}

Up to a fifth of people aged $>65$ have $\mathrm{MCl}$ and diagnosis rates in wealthier countries are rising. Nearly half of people with $\mathrm{aMCl}$, also known as $\mathrm{MCl}$ due to $\mathrm{AD}$, or prodromal $\mathrm{AD}$, develop dementia in three years. This time is a potential intervention window to delay its onset and reduce incidence and prevalence, although there are no current effective interventions. There is evidence from longitudinal studies that addressing diabetes may help reduce conversion from $\mathrm{MCl}$ to dementia. Multimodal and multicomponent interventions targeting heterogeneous causes of progression to dementia in people at risk of dementia (not necessarily with $\mathrm{MCl}$ ) may reduce risk of cognitive decline but have not been trialed in $\mathrm{MCl}$ specifically. Any intervention developed to reduce the progression to dementia from $\mathrm{MCl}$ will need to be practical and replicable so it can be scaled-up. ChEls are not effective in $\mathrm{MCl}$ and should not be used.

\section{Diagnosing}

\section{Increasing the diagnosis rate}

There are now public health strategies and plans to increase the diagnosis rate for dementia in many countries, including Bulgaria, Denmark, France, Israel, Malta, Netherlands, Norway, Switzerland and the UK. The English strategy was instituted after variations in the diagnosis rate across regions of 
England were highlighted (226). It consisted of three parts. Firstly, a public and practitioner information campaign, including TV and newspaper adverts to counter the argument that a diagnosis of dementia was not worthwhile, rooted in the mistaken beliefs that dementia is inevitable as we age and that no treatment or support is available. The second part was to provide practitioners with the confidence and tools to make a diagnosis and increase the number of diagnostic memory clinics (227). Thirdly, there was a monitoring and targeting of diagnosis rates at primary care level; a "quantified ambition" to reach a two-thirds diagnosis rate. Since this has started there has been diagnostic rates increases in in the UK, with a concomitant increase in the prescription of anti-dementia drugs, so that rates went up from an initial base of less than $40 \%$ in 2009 to 50\% in March 2014 to 67\% in November 2015 (228) (229).

\section{Screening or case finding for dementia}

Screening all older people for dementia is not recommended because of unclear benefits (230). However, case finding, i.e. searching systematically for people at high risk, may be appropriate considering that a disproportionate number of people with dementia are admitted to hospital as an emergency for physical ill-health prior to dementia being diagnosed, so that possibly $40 \%$ of older people in hospital have dementia (231). These hospital admissions typically lead to poorer outcomes and longer admissions than for people with similar physical problems but without dementia possibly because people may be treated without recognition that they lack capacity to consent to treatment or be discharged home without additional support for complex medication regimes and without participating in or understanding the discharge plan (231;232). Clinicians should therefore consider case finding in older people admitted to hospital to improve their management and outcomes.

\section{Timely detection of dementia}

A timely diagnosis, meaning communicating a diagnosis at a time when the person with dementia and their carers will benefit from interventions and support, is a prerequisite for good dementia care. Many people with dementia are never given the diagnosis (233), only 20-50\% of those with dementia have a primary care notes recorded diagnosis, and this number is less in lower-income countries (234). Many receive a diagnosis when it is too late for them to make decisions about their own and their family's future; or to benefit from interventions. Although some people do not wish to know the diagnosis, people with dementia and their families find diagnostic uncertainty anxietyprovoking and are often relieved by diagnostic certainty (235-238). Yet diagnosis is often delayed for several years, resulting in increased anxiety and carer burden in the interim (236). Timely diagnosis allows people to plan for the future; decide to have experiences they would otherwise delay; benefit from treatments; and access social support and voluntary care. These interventions can reduce or delay the progression of cognitive and neuropsychiatric symptoms (239) and decrease crises by, for example, supporting people to pay bills and take prescribed medication and delay care home entry. Additionally, knowing there is a diagnosis helps families understand their relative's behaviour and allows them to access evidence-based therapies (discussed in more detail later) which improve coping skills, reducing their high risk of developing affective disorders (240-242). There are few adverse effects of diagnosis and most people say they would want to know if they had developed dementia (243).

Timely diagnosis is often difficult for a variety of reasons (236), such as people considering the symptoms are an inevitable part of ageing, people with memory problems being reluctant to consult their GP about their memory or denying problems when seen (237), possibly related to fear of the 
diagnosis and concerns about stigma (244) as well as lack of insight. GPs may be reluctant or unsure how to make this diagnosis (245) and may not include cognitive evaluation for older adults as part of routine patient management. The short time reported in a cohort between initial recorded diagnosis and death suggests diagnosis is frequently made late and at a time of crisis (246). Later diagnosis is a particular problem for those from Minority Ethnic groups, where stigma and a lack of understanding that dementia is an illness can be especially problematic (247) and where there may be poor access to or lack of acceptance of medical care (248).

A systematic review of trials to increase dementia diagnosis rates found no clearly successful intervention (249). Although educating GPs increased their ability to diagnose dementia, this did not increase diagnostic rates in practice and local campaigns were ineffective on their own. A casefinding approach in primary care, where patients and families are asked about concerns about their memory and intent to act on them, may delineate a group who are more likely to have dementia (250). A recent intervention to increase timely diagnosis by empowering patients led to an increase in patients presenting to the GP but no change in the rate of referral to dementia diagnostic services (251).

\section{Key points and recommendations}

Diagnosis of dementia is a vehicle to improve care but is often delayed. While screening for dementia is not recommended, clinicians should consider case-finding in high-risk groups. Successful strategies to increase diagnosis to date have been at the level of public health policy and include the public and healthcare practitioners, as strategies aimed just at practitioners have been disappointing.

\section{Making the diagnosis}

National guidelines in many countries recommend that people with suspected dementia are referred to a specialist memory clinic or individual specialist doctor $(233 ; 252)$. Guidelines recommend a systematic approach: history taking from the patient and informant, review of medication, structured cognitive assessment, blood tests and (in some countries) structural imaging. The blood tests are to detect comorbid illness whose treatment may improve cognition, and the very rare reversible dementias, such as that caused by hypothyroidism or infection, e.g. syphilis or Human Immunodeficiency Virus (253).

Imaging can be either computed tomography (CT) or magnetic resonance imaging (MRI) and its purpose is to exclude rare treatable causes and to elucidate the aetiology, allowing pharmacological and psychosocial treatments to be tailored to the specific dementia subtype.

\section{Cognitive testing}

There are large numbers of short validated cognitive tests, with a recent systematic review identifying 22 (254) so professionals have to consider which to use and interpret the results, taking into account the setting and the individual patient's premorbid education, language and literacy skills and any current motor, hearing and visual impairment. The most commonly used test is the Mini-Mental State Examination (MMSE) (255) but it lacks sensitivity in patients with high premorbid educational attainment and suspected early impairment and intellectual property rights limit its broad use internationally (256). The short form of the Addenbrooke's Cognitive Examination (ACE-R or its equivalent ACE-III), available in a many languages, is more sensitive $(254 ; 257)$. The shorter 
forms of the ACE and Montreal Cognitive Assessment are also effective in detecting dementia with Parkinson's disease or DLB (258;259). The Rowland Universal Dementia Assessment scale (RUDAS) (260) is useful when literacy or education is low. Computerised assessments are likely to be used more often in the future.

\section{Neuroimaging}

Most national guidelines suggest that structural neuroimaging are part of routine clinical assessment of dementia, though in many areas there is no feasible access to neuroimaging, and some countries, e.g. Canada (261), do not recommend its routine use. CT scans are cheaper, quicker (helpful if patients have trouble lying flat or remaining still) and can be used in those with pacemakers (262). However, MRI is the preferred imaging modality for early diagnosis due its greater sensitivity and ability to differentiate dementia subtypes, especially for those with vascular lesions.

\section{Structural imaging: regional and progressive brain atrophy}

The pattern of regional brain atrophy helps in distinguishing the common neurodegenerative causes of dementia e.g. FTD from AD (263). Disproportionate hippocampal atrophy suggests AD over vascular dementia or DLB but there is overlap (264). Rates of brain atrophy on serial MRI are increased (3-4 times) in AD relative to age-matched control individuals (265)(266). A repeat scan after a year may clarify the diagnosis, distinguishing changes from natural morphological variation.

Medial temporal lobe atrophy on MRI also differentiates AD from healthy ageing; as a result these findings have been incorporated into new research diagnostic criteria for $A D$, prodromal $A D$ and $M C l$ due to $A D$ (181). MRI also differentiates AD from vascular dementia or DLB with more than $80 \%$ sensitivity and specificity and is predictive of progression from $\mathrm{MCl}$ to $A D$ with almost the same level of accuracy $(267 ; 268)$.

\section{Vascular abnormalities}

Evidence of significant vascular burden on imaging is a prerequisite for a diagnosis of vascular dementia. Significant vascular burden is defined as either many lacunae, strategic infarcts, a substantial burden (>25\%) of white matter lesions, or a combination of these (269). The degree of vascular pathology has to credibly account for the clinical cognitive impairment (269) as some degree of vascular change is typical in older populations without dementia and therefore is also present in other forms of dementia (270). As AD and cerebrovascular disease commonly co-exist, it is often difficult to ascribe accurately the relative contributions of each to an individual's cognitive decline. However, clinicians should ensure that substantial change, as described above, is present if the dementia is to be attributed entirely to vascular pathology.

\section{Functional and Molecular Imaging}

Positron emission tomography imaging using fludeoxyglucose (18F) as radiotracer (FDG-PET) permits in vivo assessment of brain metabolism and supports assessment of FTD, particularly when clinical evaluation is uncertain and there is little change on structural imaging. It shows focal frontal and/or temporal hypometabolism which is characterised by temporoparietal and posterior cingulate hypometabolism $(181 ; 271)$. Therefore in the US the use of FDG-PET for differentiating FTD from AD is 'reimbursable' by Medicare in patients who meet diagnostic criteria for both AD and FTD(272). FDG-PET has greater accuracy than imaging of cerebral perfusion with hexamethylpropyleneamine oxime SPECT $(273 ; 274)$. 
Functional imaging is helpful clinically in distinguishing DLB from other causes of dementia as dopamine depletion can be detected by DAT (dopamine transporter) scans $(275 ; 276)$. In moderate dementia when DLB is suspected, a normal DAT scan reliably excludes DLB although at early stages there is a $20 \%$ false negative rate (277).

Molecular imaging of amyloid or tau is a major research advance and is a promising modality for diagnosis of AD with several amyloid PET tracers currently licensed for clinical use(278). Published "appropriate use criteria" suggest amyloid PET imaging is most appropriate where there is diagnostic uncertainty about possible AD after expert evaluation (279) and is most helpful for young-onset or unexplained progressive dementias. Cerebral amyloid plaque accumulation in $A D$ is thought to precede clinical symptoms by more than a decade which gives amyloid PET high sensitivity but relatively low specificity in older individuals. Although widely used in research, clinical use of amyloid imaging is limited by its cost in the absence of a disease-modifying treatment and uncertainties about the risk of false positive AD diagnoses. Tau imaging is currently only a research tool $(279 ; 280)$.

MRI incorporating diffusion imaging has great sensitivity and specificity for prion disease which is a rare cause of dementia; typical changes are virtually pathognomonic (263).

\section{Cerebrospinal fluid and blood biomarkers}

Routine testing of cerebrospinal fluid (CSF) or blood for biomarkers is not currently recommended clinically by any national guidelines, though the American Academy of Neurology recommends CSF testing for investigation of younger patients with dementia (281) and the European Federation of Neurological Societies recommend its use in atypical clinical presentations of AD (282). However, there is interest in the future value of such tests as they have the potential to elucidate the dementia subtype at an earlier stage, as CSF changes supportive of a diagnosis of AD can be identified up to 15 years before the clinical presentation of dementia $(195 ; 283)$. There are wide variations in current practice globally, varying from routine use in the Netherlands and Sweden, where $40 \%$ of people with newly diagnosed dementia had a lumbar puncture (284), to infrequent use in North America, where it is reserved for research settings with strict protocols (285), reflecting uncertainty about the added value of these investigations, as heightened diagnostic accuracy does not translate to tailored drug treatments.

However, there is little doubt that analysing biomarkers improves AD diagnostic accuracy and may in future be markers of disease progression or outcome targets for clinical trials. There is a large number of potential biomarkers which represent neurodegeneration, amyloid precursor protein metabolism, tangle pathology, blood-brain-barrier function or glial activation due to inflammation (286). However, results tend to be from highly selected populations, that even a meta-analysis of many studies may produce overly optimistic performance results. There can also be reproducibility and accuracy difficulties in the measurement of amyloid (but not tau) biomarkers. A recent comprehensive meta-analysis (287), of 15 potential biomarkers across 231 studies, found that elevated levels of CSF T-tau (average ratio for AD versus control was $2 \cdot 54,95 \% \mathrm{Cl}$ [2.44-2.64]) and Ptau (1.88, 95\% Cl [1.79-1.97]) and low CSF A 42 (0.56, 95\% Cl [0.55-0.58]) differentiated between people with $A D$ and healthy controls. A similar pattern distinguished between people with $\mathrm{MCl}$ who go on to develop $A D$ and those who do not (average ratio 1.76 for T-tau, 1.72 for P-tau, and 0.67 for CSF A 42 ). Other biomarkers studied had limited value, except for CSF Neurofilament light protein (2.35, 95\% Cl [1.90-2.91]) and plasma T-tau (1.95, 95\% Cl [1.12-3.38]) (287). 
No specific fluid biomarkers exist or are clinically recommended for DLB or the frontotemporal dementias in general, but the above approaches may differentiate these forms of dementia from $A D$ $(286 ; 288)$. Specific genetic variants of frontotemporal dementia can be identified using plasma and CSF biomarker testing, such as by reduced CSF and plasma levels of the protein progranulin (288) in people with progranulin gene (GRN) mutations (289), but accurate prognosis or differential treatment of these FTD subtypes is not yet developed enough for clinical value. Dementia caused by rapidly-progressive prion disease is rare but may be detected with high sensitivity and specificity using CSF biomarkers (290) (286).

CSF biomarker analysis has the potential for adverse consequences. There are direct risks of pain, anxiety and post-lumbar puncture headache (285), and cost implications, although the only costeffectiveness analysis judged it to be, at $€ 205$ (approximately $£ 173$ or USD\$230), a cost-effective investigation for diagnosing likely $A D$ in $\mathrm{MCl}$ (291). Diagnosis may also be delayed by additional investigations, a situation which would be exacerbated by more widespread use.

Further research into the predictive value of fluid biomarkers and the development of standardised analytic techniques and normal laboratory ranges is needed $(286 ; 288 ; 292)$. Previous guidelines suggested that CSF analysis should be reserved for when rare reversible causes of cognitive decline are suspected (293) (if a history of metastatic cancer, suspicion of CNS infection, reactive serum syphilis serology, hydrocephalus, age under 55 years, rapidly progressive or unusual dementia, immunosuppression or suspicion of vasculitis) and updated diagnostic criteria for AD suggest that CSF analysis should not be routine (292).

\section{Genetic testing}

Genetic contributions to dementia are complex and genetic testing is not recommended for all due to ethical concerns about uncertain benefit and potential harm. The ApoE $\varepsilon 4$ allele is the only genetic factor which greatly increases susceptibility to late-onset AD (onset age $>65$ years) (294). It increases dementia risk, with heterozygotes having a three times higher risk and homozygotes risk being 15 times higher than that of ApoE $\varepsilon 3$ homozygotes (295). As ApoE $\varepsilon 4$ does not alone cause AD, testing for the allele is not clinically recommended (282).

Young-onset familial AD is linked, in 50\% of cases to mutations in the APP, PS1 or PS2 genes (296). Several contributory genes for the frontotemporal dementias have been identified, including GRN, microtubule-associated protein tau (MAPT) and C9ORF72. Again, the clinical implications of these specific diagnoses are not sufficiently clear for routine testing (297).

Testing of patients and unaffected at-risk relatives for genetic causes of dementia is not routinely done and should only be conducted with fully informed consent, after genetic counselling.

\section{Key points and recommendations}

Diagnosis requires structured history taking, cognitive tests and blood screening. Results of cognitive testing should be interpreted in the light of premorbid education, language and literacy skills and any current motor, hearing and visual impairment.

We recommend structural neuroimaging for suspected $A D$ and vascular dementia with MRI, if available. For those who cannot tolerate MRI, CT imaging should be used, and if possible comment on hippocampal volume. Vascular changes often co-exist with AD but a diagnosis of vascular dementia requires demonstration of major infarcts, a substantial burden $(>25 \%)$ of white matter 
lesions or many lacunae or strategic infarcts. Functional imaging of dopamine is helpful for distinguishing Lewy body disease from AD.

CSF testing for dementia-related biomarkers is not routinely used in most countries. In most countries it is reserved for the exclusion of rare reversible causes of dementia or for possible youngonset dementia.

\section{Treating}

\section{Principles of assessment and treatment in people with dementia}

People with dementia have complex problems, as they have symptoms in many domains. These include cognition, neuropsychiatric symptoms, activities of daily living and usually comorbid physical illnesses. Interventions have to consider the person as a whole and attend to their medical, cognitive, emotional, psychological and social needs. Thus, individuals require different treatments and these will change with the course of the dementia. Assessment of an individual's problems in these areas is termed 'needs assessment' (298).

Everyone with dementia should have their physical health including medication reviewed, a risk assessment, management plan and interventions to maximise cognition. We have taken the clinical approach below of considering individual needs in cognition, psychosis, agitation, depression, sleep and apathy and then discuss possible approaches to management; psychological, social, environmental, physical and medication. We have drawn algorithms to help navigate these complex plans. All are consistent with the multi-disciplinary DICE approach for the assessment and management of neuropsychiatric symptoms of dementia (Describe the problem, Investigate the cause, Create a plan and Evaluate the effectiveness of it) (299) which can be used as a general approach. After the sections on what treatments to use, we have discussed their delivery.

\section{Principles of psychological, social and environmental management}

There are around 100 RCTs published in the last ten years with intermediate (not high) level evidence about outcomes in dementia (300). In this section we address the evidence for management strategies for specific syndromes, like depression or agitation. We discuss in the next sections those aimed at helping family carers. While interventions are diverse, many follow a consistent pattern. The most effective psychosocial treatments are usually multimodal, individualise care and train carers in skills including optimising communication, coping and environmental adaptations (301). There is no "magic bullet" in the treatment of dementia, that is treatments that target all symptoms with one type of intervention, either pharmacological or non-pharmacological, do not work. All treatments require that target symptoms are defined and measured.

Such strategies and programmes involve more than 'being nice' or 'providing advice'. Rather, those that show the best results are structured and systematic. Some have published manuals and materials available to professionals working with carers and people with dementia (242;242;302306). Many other approaches have been tried and not worked, so it is important to use evidencebased strategies.

\section{Risk assessment and management}

Part of the initial assessment of all people with dementia is to evaluate and manage risk, to enable people with dementia to live well at home, for as long as possible. The risks change throughout the 
course of dementia and therefore require regular re-assessment. Most societies place a high ethical value on autonomy (307). Therefore risk management must balance a person with dementia's rights with society's and families usually beneficent wishes to reduce risks. The general principle is of risk enablement, to allow people to have an acceptable level of risk, managed by using the least restrictive options (308). This requires an assessment of the decisional capacity of the person with dementia regarding risks (discussed further below). The risks which should be considered arise mainly because of decreased ability to maintain safety, through forgetting, apathy, decreased insight or poor judgement. These include, but are not limited to, nutritional deficiencies resulting from being unable to plan to eat and drink well; not being able to understand or remember to take medication as prescribed; lack of safety at home through falls, floods, fire or gas escape, with subsequent risks to other people; poor road safety both in walking and in driving; and potential vulnerability to crime and abuse from others (309-311).

Removing means of serious harm, including access to guns for people with dementia and carers who have thoughts of causing harm, would be a practical way of protecting from harm. Preventing people with dementia who cannot drive safely from doing so protects people with dementia, carers and society; there are country-specific rules about driving.

Family, friends or care professionals frequently manage other risks on an everyday basis. They use simple measures such as ensuring vulnerable people with dementia are not left alone in risky situations, prompting to eat, using automatic alarms for heat, smoke, gas or movement and wearing alert bracelets with contact details. There are also legal measures, such as a family member being nominated as an attorney, so that families can pay bills and manage money and these are discussed further in the family carers' section. Medication should be simplified and can be packaged in easy-tomanage forms (blister packs, dossette boxes) and family, services or technology can remind people to take them. The following sections address these in more detail, including how to offer support and assess capacity to make decisions, and potential technological approaches.

\section{Cognition}

\section{Medication for cognition}

The only approved drug treatments in many countries for cognitive symptoms of dementia are for AD, DLB or Parkinson's disease dementia (PDD). They target biochemical abnormalities consequent upon neuronal loss but do not modify the underlying neuropathology or its progression. ChEls may partly restore the deficit in acetylcholine arising from loss of neurones in the nucleus basalis of Meynert and in the central septal area, projecting to cortical regions (312). Memantine may attenuate the toxic effects of glutamate released from degenerating neurones, although it is uncertain that this is its mechanism of action (313). No neuroprotective potential has been demonstrated in humans (314). Few studies of anti-dementia drugs provide placebo-controlled data beyond six months. They are not indicated in $\mathrm{MCl}$ as people with prodromal $\mathrm{AD}$ did not show clinically meaningful improvement or slowing of progression in trials of ChEls, and systematic reviews of $\mathrm{MCl}$ trials suggest increased mortality risks $(315 ; 316)$.

\section{Cholinesterase inhibitors}

Three ChEls are in routine use: donepezil, rivastigmine and galantamine. These are available respectively as a tablet or orodispersible tablet; transdermal patch or capsule or liquid; and as a 
capsule. Most evidence about these three drugs for AD is summarised in the 2006 review from the Cochrane collaboration (317). All ChEls at optimal doses, compared to placebo, show modest benefit on cognition as measured by the Alzheimer's Disease Assessment Scale -cognition (ADAS-cog) of 2.4 points (318). They also show a mean difference of 1.37 points on MMSE (figure 6) which is equivalent to the minimum clinically important difference (319). Studies published since 2006 confirm ChEls' cognitive benefit of (320-322). There are also benefits in global change, assessed by clinician with carer's input (figure 7), and activities of daily living. An updated Cochrane review about rivastigmine in $A D$ found a similar but slightly smaller effect (323). The very small difference in behavioural symptoms on the neuropsychiatric inventory (NPI) (324) (mean difference $-2.44,95 \% \mathrm{CI}$ [-4.12 to -0.76]) is not a clinically-significant difference. Though these studies do not exclude people, they have not purposively recruited participants with neuropsychiatric symptoms, so this finding may be limited to people with relatively minor symptoms. We report ChEl's effect in specific syndromes later in this report.

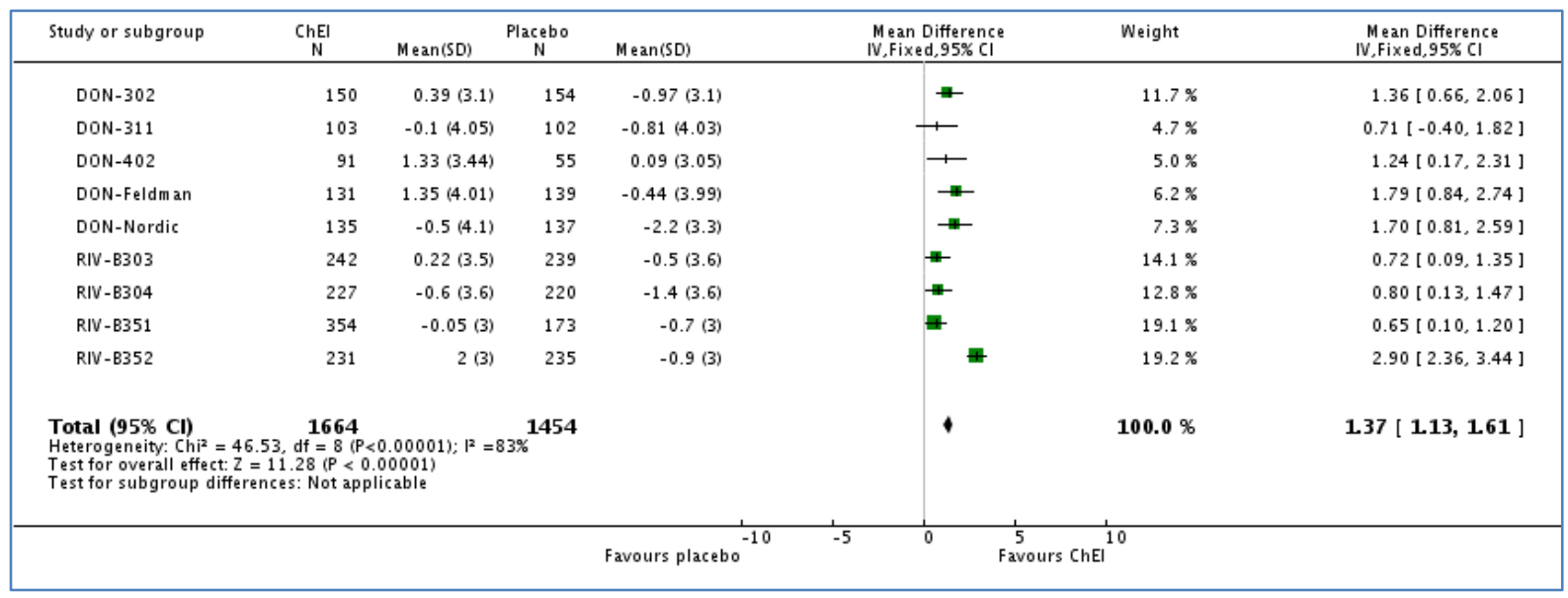

Figure 6. Effect of cholinesterase inhibitors at optimum dose on cognition, measured by MMSE, in Alzheimer's disease; mean change in score from baseline at 6 months or later

Figure reproduced from Birks et al (317)by permission of the Cochrane Database of Systematic Reviews

Key: $D O N$ - = donepezil trial; $R I V$ - = rivastigmine trial

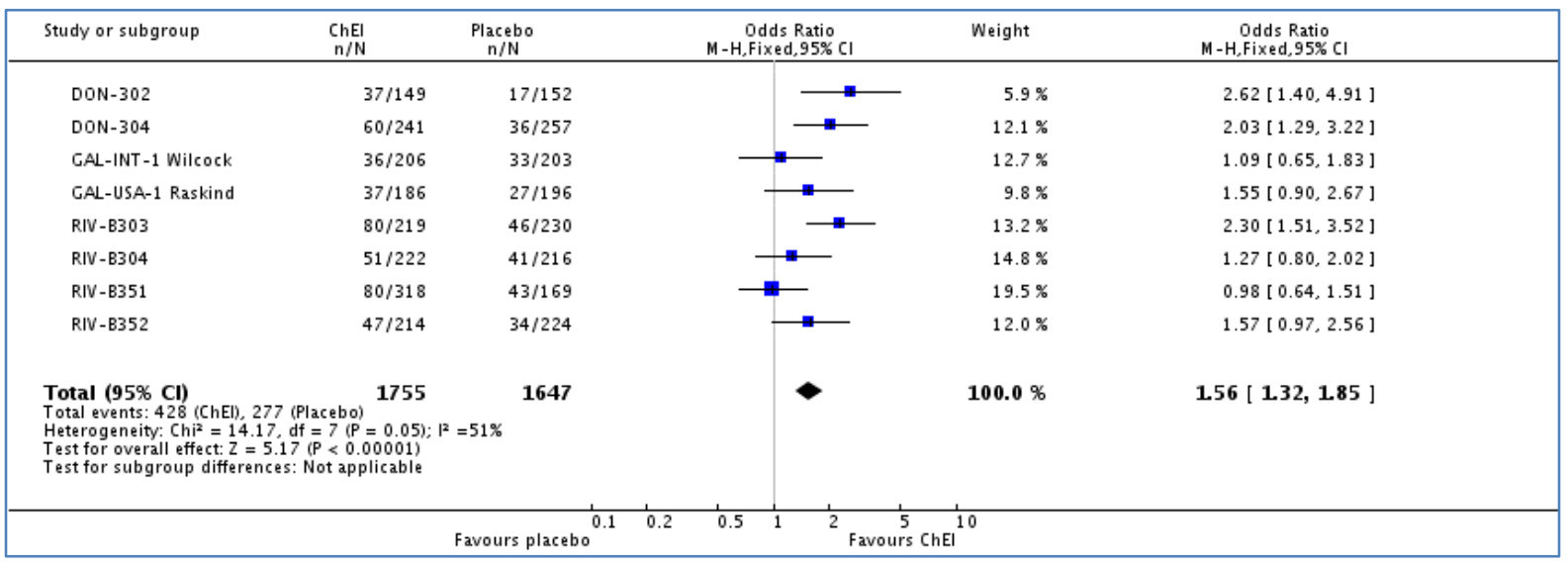


Figure 7. Effect of cholinesterase inhibitors at optimum dose on global assessment, measured by CIBIC-Plus, in Alzheimer's disease; mean change in score from baseline at 6 months or later

Figure reproduced from Birks et al (317) by permission of the Cochrane Database of Systematic Reviews

Key: DON- = donepezil trial; GAL- = galantamine trial; RIV- = rivastigmine trial

ChEls are sufficiently clinically- and cost-effective for NICE to recommend any of them for managing mild-to-moderate $A D$ (325). It is not currently possible to assess who are responders based on their initial response to medication, so treatment should continue if the patient agrees to and tolerates the medication. The ChEls are fairly well tolerated but adverse events seen in patients taking such medications include nausea, vomiting, diarrhoea, vivid dreams (reported for donepezil only, and ameliorated by morning dosing) and leg cramps, and RCTs report higher withdrawals due to adverse events in those taking ChEls than placebos.

As trials of ChEls have not usually continued over years, it was previously unclear if ChEl treatment benefits continued as AD progressed. However the DOMINO trial, a well-conducted double-blind discontinuation study, found that donepezil cessation (replaced by a placebo) in those with moderate-to-severe AD (MMSE <12) was accompanied by a cognitive (MMSE mean difference = 1.9) and functional decline, an increase in neuropsychiatric symptoms and doubling of risk of care home admission in the year after discontinuation (326) (327). These results suggest ChEls should be continued for people whose dementia has become severe.

The potential for greater benefit from higher ChEl doses is theorised from imaging showing that $10 \mathrm{mg}$ of donepezil resulted in inhibition of only $19-27 \%$ of cerebral cortical acetylcholinesterase activity (328;329). A double-blind RCT on 1371 people with moderate-severe AD found that, after 24 weeks, those taking a $23 \mathrm{mg}$ donepezil tablet per day scored 2.2 points higher on the 100 point Severe Impairment Battery than those continuing to take $10 \mathrm{mg}$ daily. There was no difference in clinician assessment of overall severity and functioning and more people in the high-dose group (18.6\% versus $7.9 \%$ ) withdrew from the study due to adverse events, most commonly gastrointestinal (330). Post-hoc analyses suggested greater benefit of high-dose donepezil for severe dementia but this was not replicated in a study, which found no significant difference between $10 \mathrm{mg}$ and $23 \mathrm{mg}$ donepezil tablets in severe dementia (331). While the US FDA has licenced a $23 \mathrm{mg}$ donepezil tablet, and it is used in the US in later stages of $A D(332)$, the clinical-effectiveness remains uncertain. Rivastigmine 24 hour patches come in doses of $4.6 \mathrm{mg}, 9.5 \mathrm{mg}$ and $13.3 \mathrm{mg}$. The OPTIMA trial $(333 ; 334)$ found that the $13.3 \mathrm{mg}$ patch was better than the $9.5 \mathrm{mg}$ patch for ADL (at week 48) and cognition (at week 24 ) in people with mild-to -moderate AD.

ChEls are also used for DLB and both rivastigmine $6-12 \mathrm{mg}$ and donepezil 5 and $10 \mathrm{mg}$ have been found in double-blind, placebo-controlled trials to be safe and well tolerated, with a cognitive effect, and a reduction in visual hallucinations $(335 ; 336)$. Meta-analyses find that ChEls improve cognition and global function in DLB and PDD (337;338). Only the largest of four trials assessing behaviour showed a nominally significant, and very small, effect on behaviour. ChEls or memantine are not recommended for vascular (339) or frontotemporal dementias (340). 


\section{Memantine}

Memantine is a non-competitive modulator of the NMDA receptor and normalises glutamatergic neurotransmission. It prevents excitatory amino acid neurotoxicity (341). It is usually given up to a dose of $20 \mathrm{mg} /$ day. A meta-analysis summarised three trials on 1291 patients with moderate-tosevere $A D$ (MMSE 3-14) and three unpublished studies in 997 patients with mild-to-moderate $A D$, all lasting six-months (342). In the moderate-to-severe group, there was a small beneficial effect on cognition (figure 8 ), activities of daily living, mean levels of neuropsychiatric symptoms and global assessment (mean difference on CIBIC-plus $0.28,95 \% \mathrm{CI}[0.15-0.41]$ ). In the mild-to-moderate groups, there was a marginal beneficial effect on cognition which was not accompanied by effects on behaviour or everyday functioning.

Two trials of memantine in mild-to-moderate DLB found improvement in global impression (343;344), and one of these found improvement in mean behavioural symptoms (343), but no benefit in other clinical domains. A marginal benefit for cognition in mild-to-moderate vascular dementia did not equate to any global or functional improvement.

\begin{tabular}{|c|c|c|c|c|c|c|c|}
\hline Study or subgroup & Memantine & Mean(SD) & Placebo & $\operatorname{Mean}(S D)$ & $\begin{array}{l}\text { Mean Difference } \\
\mathrm{IV}, \text { Fixed, } 95 \% \mathrm{Cl}\end{array}$ & Weight & $\begin{array}{l}\text { Mean Difference } \\
\text { IV,Fixed,95\% CI }\end{array}$ \\
\hline 9605/Reisberg 2003 & 124 & $-4(11.34)$ & 123 & $-10.1(13.5)$ & - & $17.2 \%$ & $6.10[2.99,9.21]$ \\
\hline MD-02/T ariot 2004 & 198 & $0.9(9.42)$ & 196 & $-2.5(9.66)$ & 7 & $46.9 \%$ & $3.40[1.52,5.28]$ \\
\hline MD- 01 & 170 & $-1.7(11.34)$ & 165 & $-2.6(8.61)$ & -1 & $35.9 \%$ & $0.90[-1.25,3.05]$ \\
\hline \multicolumn{3}{|c|}{ 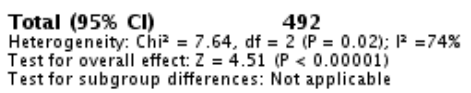 } & 484 & & $<$ & $100.0 \%$ & $2.97[1.68,4.26]$ \\
\hline & & & & Favours placebo ${ }^{-10}$ & ${ }^{0}{ }^{\circ}$ Favours & $e^{10}$ & \\
\hline
\end{tabular}

Figure 8. Effect of memantine at optimum dose on cognition, measured by the severe impairment battery, in moderate to severe Alzheimer's disease; mean change in score from baseline at 6 months or later

Figure reproduced from McShane et al (342) by permission of the Cochrane Database of Systematic Reviews

A consensus panel made a tentative positive recommendation for the benefit of a combination of memantine and ChEls in moderate-to-severe AD on the basis of a recent meta-analysis showing small but significant benefit for global assessment, cognitive ability and neuropsychiatric symptoms without major differences in adverse events rate (345). The single study considering the combination of high-dose rivastigmine patch $(13.3 \mathrm{mg} / 24$ hours) and memantine for severe $A D$ found no additional therapeutic benefit but that this combination was safe (346).

There are no controlled data on the memantine's efficacy beyond six months and no studies to determine whether it can delay progression from $\mathrm{MCl}$ to dementia. Memantine is an option for managing moderate $A D$ for people who cannot take ChEls, and for managing severe $A D$ (325). An extended release formulation of memantine at a higher dose of $28 \mathrm{mg}$ daily is licenced in the US for moderate-to-severe $A D$ and has a more convenient dosing schedule. A placebo-controlled trial found it was effective in people with moderate-to-severe $A D$ (347) but the observed effects were not larger than those of the standard formulation at lower doses and no direct comparison has taken place. 


\section{Souvenaid}

Souvenaid is a medical food product for oral consumption formulated to meet nutritional requirements in $A D$ and comprises docosahexaenoic acid (DHA), eicosapentaenoic acid (EPA), uridine-mono-phosphate (UMP), choline, phospholipids, folic acid, vitamins B6, B12, C, E, and selenium. These are hypothesised to be useful as precursors and co-factors for the formation of neuronal membranes and consumption of souvenaid increases their levels $(348 ; 349)$. However, a double-blind trial of 527 participants with mild-to-moderate AD showed no difference in the ADASCog outcomes (350). A systematic review and meta- analysis found good quality studies with a total of 1011 participants and no difference in global cognition, functional levels or behaviour (351).

\section{Key points and recommendations}

Cholinesterase inhibitors (donepezil, rivastigmine and galantamine) have a small but clinically important effect on cognition and function at all AD severities but have side-effects. Donepezil and rivastigmine have a positive effect on cognition, and in the Lewy body disorders, on hallucinations. Memantine has a smaller effect on cognition in moderate-to-severe AD.

\section{Other cognitive interventions}

Cognitive interventions encompass a range of approaches to maintain or improve cognition through mentally stimulating activities. There are broadly three main cognitive intervention approaches.

Cognitive stimulation therapy (CST) stems from reality orientation and is usually group-based. It consists of group sessions led by a trained coordinator incorporating social activity, reminiscence and simple cognitive exercises (Box 2). Cognitive rehabilitation aims to improve everyday function by helping the patient set individual goals, and devising strategies to achieve these (352) and may be useful for patients with mild-to-moderate $A D$, for whom individualised goals to improve specific functions could improve function and quality of life. A large multicentre study of goal orientated cognitive rehabilitation in mild $A D$ is currently underway (353). Cognitive training involves theoretically-driven strategies or exercises targeting specific cognitive domains, usually with an adaptive level of difficulty. Cognitive training may have benefits in healthy older adults but not for those with $\mathrm{MCl}$, as detailed above. 


\section{What is Cognitive Stimulation Therapy?}

The aim of Cognitive Stimulation Therapy (CST) is to actively mentally stimulate participants through cognitive activities and reminiscence, multi-sensory stimulation and group social contact. Each session is led by a facilitator. The standard CST model is a group intervention of 14 themed sessions, each lasting approximately 45 minutes and held twice per week. This standard programme has been manualised and can be potentially administered by anyone working with people with dementia and held in care homes, hospitals or day centres.

The programme includes:

- A non-cognitive warm-up activity (e.g. soft ball game and song).

- Elements of 'reality orientation' including a board displaying personal and orientation information.

Sessions then focus on different themes including childhood, food and current affairs, using money, faces, scenes and quizzes or word games.

\section{Box 2. Cognitive stimulation therapy}

\section{Cognitive Stimulation Therapy}

CST (354) is the psychological approach with the strongest evidence for improving cognition. Recent meta-analyses find that CST benefits general cognition (Hedges g effect size $=0.51$, equivalent to a mean difference of CST vs control of 1.78 points (95\% Cl, [1.23-2.33]) on the MMSE (figure 9) (355)), comparable with ChEls although, unlike in ChEl trials, the control group in CST trials has no placebo therapy (356). A Cochrane Review has found that CST may improve self-reported quality of life (SMD $=0.38,95 \% \mathrm{Cl}[0.11-0.66])$, but had no significant effect on ADLs (SMD $=0.21,95 \% \mathrm{Cl}[-0.05-0.47]$ ) (356). CST is cost-effective for people with mild-to-moderate dementia and is recommended in the UK by NICE. Despite the evidence of effectiveness however, limitations include a lack of active control interventions, few attempts to blind raters and limited follow-up studies to clarify how long effects last (355). The group-based and multi-component nature of CST also means it is unclear which aspects of the intervention are the most useful, and whether the social element is crucial , a distinct possibility, as individualised CST has not been found to be effective (357). Overall, while clearly efficacious the evidence that this reaches the threshold for a minimum clinically important difference is debatable, and it may not be effective in all settings. 


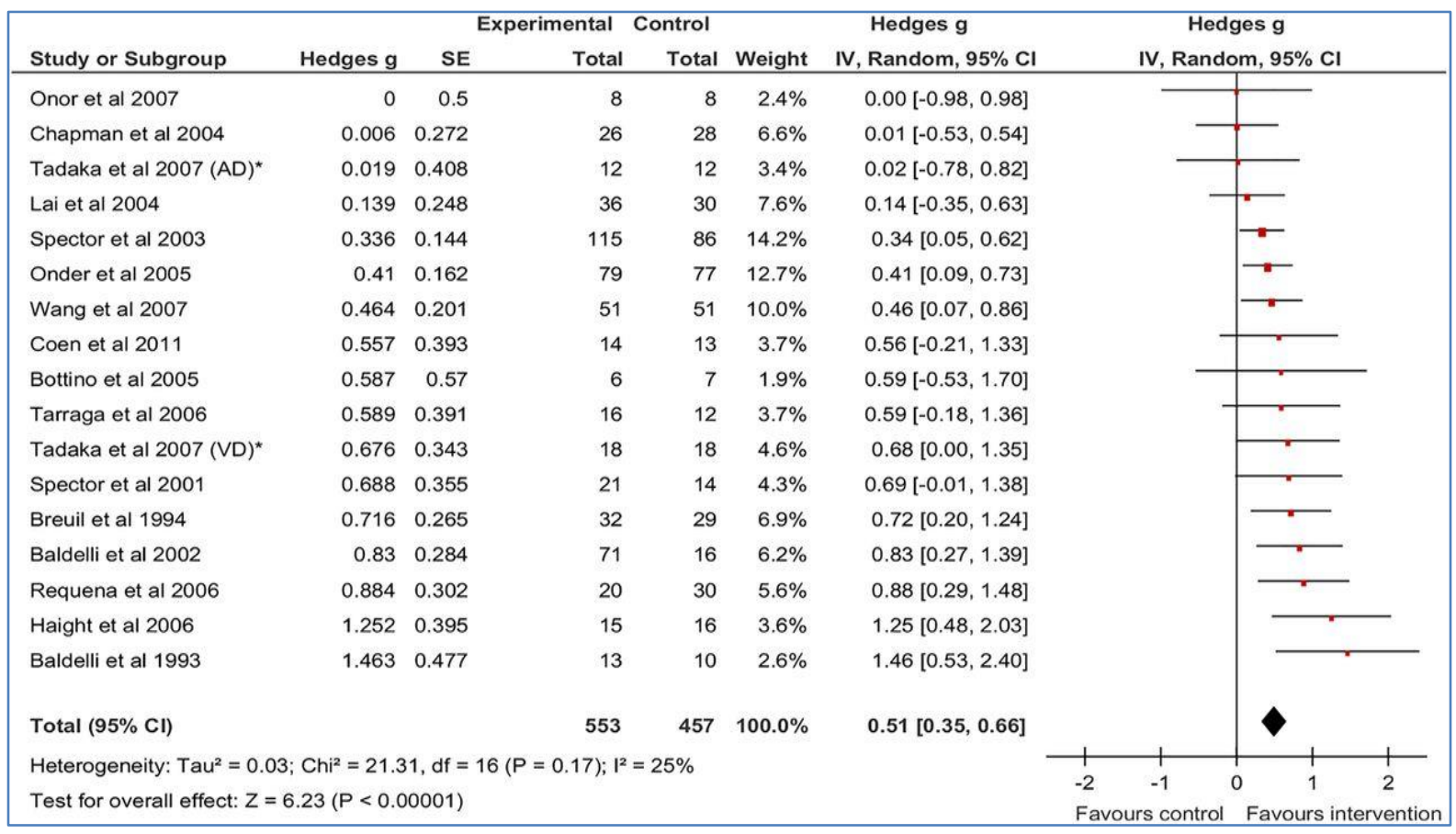

Figure 9. Effect of Cognitive Stimulation Therapy vs usual care on cognition, measured by the MMSE

Figure reproduced from Huntley et al (355) by permission of BMJ Publishing Group*needs permission

\section{Cognitive training}

There are relatively few RCTs on cognitive training in dementia, and their small sample sizes, outcome measures variability, and multiple techniques used, making it difficult to evaluate single strategies. A recent meta-analysis to evaluate cognitive training for common clinical outcomes of general cognition (MMSE and ADAS-Cog) found only four RCTs reporting them. The pooled effect sizes were small and non-significant (e.g. for the MMSE effect size of $0.22,95 \% \mathrm{Cl}$ [-0.75 to 1.18]) (355). Similarly a recent Cochrane Review including six studies with a total of 173 participants found no significant effects of cognitive training on global outcome measures or activities of daily living in patients with AD and vascular dementia (358). However, an RCT of $18 \times 30$ minutes sessions of either adaptive chunking training or a control intervention over 8 weeks for 30 patients with mild AD led to improvements in verbal and general memory and further testing of adaptive training is required (359).

\section{Cognitive rehabilitation}

There are few trials of cognitive rehabilitation in people with dementia. In a recent $R C T, 653$ patients with mild $A D$ (mean MMSE 21.6) were randomised to group cognitive training, group reminiscence therapy or individualised cognitive rehabilitation weekly for 12 weeks, then 6-weekly for 21 months. There was no reduction in rate of cognitive decline compared to usual care but the individual cognitive rehabilitation group demonstrated significantly lower functional decline at 24 months. Neither intervention (versus controls) was superior on secondary cognitive, functional or behavioural outcomes (360). 


\section{Key points and recommendations}

Group CST improves cognition in patients with mild-to-moderate dementia. It is unclear whether the active component is cognitive or social as individual CST is ineffective or whether the effect size is clinically-significant. Individual cognitive rehabilitation can be effective for patients with mild-tomoderate dementia with specific functional goals, but its cost-effectiveness requires more evidence.

\section{Exercise interventions for cognition}

The evidence from RCTs that exercise interventions improve cognitive and functional outcomes in patients with dementia is highly variable. A systematic review of four RCTs of exercise interventions in $A D$ reported a significant overall standardised mean difference (SMD) on cognitive outcomes compared to controls of 0.75 (95\% $\mathrm{Cl}$ [0.32-1.17]) (361). In contrast, a recent Cochrane review of nine studies with 409 participants did not find a significant difference and rated the quality of evidence as very low (362). The Finnish Alzheimer Disease Exercise Trial reported a year-long programme improved executive function, measured on clock drawing test, (effect size in the homebased exercise group $d=0.25(95 \% \mathrm{Cl}[0.06-0.48]$ ) vs $d=-0.10,(95 \% \mathrm{Cl}[-0.27-0.16]$ in control group), but not in verbal fluency and there were no effects in other domains (363).

However, in the Cochrane review there was an overall significant benefit of exercise on ADLs (SMD = 0.68 (95\% Cl [0.08-1.27]), in six trials with 289 participants (362). The functional benefits are illustrated by the FINALEX trial, in which 210 home dwelling patients with AD were randomised to group or tailored exercise twice a week for 1 year or to usual treatment control. Although the study was unblinded, the tailored home-based exercise group declined less on the functional independence measure at 12 months (mean change -7.1 (95\% Cl [-3.7 to -10.5])) than controls (mean change $-14.4(95 \% \mathrm{Cl}[-10.9$ to -18.0$]))(364)$.

Overall, RCTs examining exercise interventions in dementia are few and limited by small sample sizes, lack of blinding, inadequate comparator groups, variable form, frequency, duration and intensity of exercise and the use of multicomponent interventions masking the effect of an exercise component. It is possible that a dose-response relationship between exercise and cognition exists, and that high intensity exercise gives more beneficial cognitive effects (365). It has been hypothesised that there is an intensity threshold beyond which cognitive benefits become more pronounced (366). Supporting this hypothesis, a sub-analysis of the ADEX trial (367) found that highintensity training is required for cognitive improvement in mild $A D$ patients. Participants performing higher intensity exercise with $>70 \%$ maximum heart rate $(n=66)$ improved in the primary cognitive outcome vs control, whereas those doing moderate intensity exercise had no significant improvement (368).

\section{Key points and recommendations}

Engaging in exercise is helpful for a variety of reasons including cardio- and cerebro-vascular health, diabetes, obesity, strength and protection against frailty. Exercise programmes for people with mildto-moderate dementia are feasible and well-tolerated and exercise offers positive small effects on function for people with dementia but it is not clear whether they help cognition. The most persuasive evidence to date on exercise is for high intensity interventions to help cognition in mild $A D$. It is unknown whether exercise programmes that reach the aerobic fitness thresholds which affects hippocampal volume or BDNF levels in non-AD participants conveys cognitive benefits. 


\section{Neuropsychiatric symptoms}

Neuropsychiatric symptoms (NPS) in dementia are common, generally increasing with stage of dementia and affecting nearly everyone with dementia at some point during their illness $(369 ; 370)$. Although there are many different symptoms they often co-occur and there are several different models of how they cluster, for example, into affective, psychotic and other symptoms (371). They also vary with the underlying cause of dementia, with visual hallucinations being common in Lewy body dementia (372). They are frequently persistent, with one study reporting that $81 \%$ of people with NPS have symptoms 18 months later (373), although this varies according to the specific symptom - apathy and hyperactivity, (agitation, disinhibition, irritability, aberrant motor behaviour and euphoria) are particularly persistent (374). Factor analysis of cross-sectional data from the European Alzheimer's Disease Consortium has suggested four NPS sub-syndromes with overlapping symptoms: psychosis (delusion, hallucination and sleep disorder), affective (depression and anxiety), apathy (apathy and appetite disorder) and hyperactivity (375). The overlap between these symptoms highlights the need for careful assessment of symptoms and potential causes, advocated by the DICE approach (376) discussed above, and in this section, we present the best evidence supporting the management of these syndromes. We discuss providing pleasant events and maximising communication as strategies to prevent and manage agitation, as that is where there is evidence, but these strategies are inherent to providing good quality care to all people with dementia.

\section{Psychosis}

Around $18 \%$ of people diagnosed with dementia experience psychosis at any one time, with prevalence greater in moderate and more severe dementia. Psychotic symptoms tend to persist in most people for several months $(373 ; 377)$.

\section{Types of psychotic symptoms in dementia}

Delusions are the most common psychotic symptoms in people with AD. Delusions are usually simple, rather than systematised and bizarre. They commonly involve theft, abandonment, infidelity or poisoning. Misidentification symptoms - beliefs that the identity of a person, such as spouse, has been changed or replaced, the phantom boarder or misidentifications when looking in the mirror, also occur. Hallucinations are less common, and in contrast to other psychiatric disorders, are more commonly visual than auditory. Auditory hallucinations are usually sounds, individual words or phrases and rarely commenting or commanding voices. Tactile or olfactory hallucinations are uncommon. A substantial proportion of people with dementia are not distressed by their psychotic symptoms. Others are; these symptoms can be associated with family carer distress, risk of care home admission, worse general health and increased mortality (378). In AD, psychotic symptoms are associated with more rapid cognitive decline and this trajectory precedes psychotic symptoms onset (378-381).

Psychotic symptoms are prominent in DLB where well-formed visual hallucinations are a core diagnostic criterion, but seem to be less common in frontotemporal dementia (382), excepting some rare genetic forms (383). No genetic contribution to psychotic symptoms has been identified, despite familial aggregation of symptoms. Imaging techniques find grey matter volume, blood flow or glucose metabolism changes are more pronounced in neocortical regions than in temporal lobe structures in patients with AD and psychosis (384). Misinterpretations of reality by a person with 
dementia are often contributed to by sensory deprivation, vision loss, hearing loss and inappropriate sensory stimulation, and may increase the risk of psychosis (385).

\section{Principles of assessing and managing psychotic symptoms in dementia (Figure 10)}

This should start with investigating the nature and context of symptoms, primarily to determine whether psychotic symptoms (as opposed to mistaken beliefs due to memory loss) are truly present.

People with dementia are vulnerable to delirium in which psychotic symptoms can be prominent, so this should also be considered. Treating the underlying causes of delirium will often relieve symptoms. In patients who are not distressed by their psychosis, management may be limited to an explanation of the symptoms to the patient and family. If the patient agrees, social stimulation such as participation in clubs and centres, and treatment of visual or hearing problems by better lighting, ophthalmological treatments, removing ear wax or using hearing aids, sometimes help. Discussion of the risks (detailed in the next section) and benefits of antipsychotic treatment will often lead to the conclusion that they are not indicated (386). In DLB, where antipsychotics are more likely to cause side-effects, rivastigmine (or donepezil) $(335 ; 336)$ are helpful for visual hallucinations but antidepressants galantamine, do not seem to be effective $(335 ; 387)$.

\section{Antipsychotic use in dementia}

Antipsychotics may cause particular harm in dementia; side effects include sedation, extrapyramidal symptoms and increased risk of cerebrovascular events and mortality $(388 ; 389)$.

\section{Harmful effects of antipsychotics in dementia}

People taking antipsychotics have higher mortality rates (22.6\%-29.1\%) than those taking other psychotropic medications (14.6\%), except for anticonvulsants (390). Concerns began in 2002 (391). The US Food and Drug administration issued a "black-box warning" about atypical antipsychotics in 2005, expanded to include first generation or conventional antipsychotics in 2008. Mortality on typical antipsychotics including haloperidol appears to be up to twice that of risperidone with greater risk at higher doses (388;392-394). Those who have been recently started on antipsychotics seem to be particularly at risk, especially in the first 30 days $(394 ; 395)$.

In the US, antipsychotic prescription began to reduce before the official warning and then decreased more sharply from 2005 to 2007 (391). In 2009, in the UK, it was calculated that two-thirds of the 180,000 people with dementia who were prescribed these drugs may not need them and their administration was related to an estimated 1800 excess deaths (or 1\%) and 1600 excess strokes annually (396). The UK Call to Action campaign mandated the recording of the number of people with dementia on antipsychotics, discussions about their use with family and carers, consideration of alternatives and 3-monthly review. An audit of practice in 2012 showed a large reduction in prescribing along with an increase in the dementia diagnosis rate (397).

More recently a meta-analysis of RCT of risperidone in dementia patients (1,009 risperidone, 712 placebo) found lower relative risk of cerebrovascular events in those treated with risperidone who had depression or delusions associated with dementia, compared to those without; and a reduction in relative risk of death in those with depression (398). Antipsychotics cause more cognitive impairment than placebo $(399 ; 400)$. In most people with AD, the adverse effects of conventional antipsychotics and the newer atypical anti-psychotic medication offset their benefits (401). 


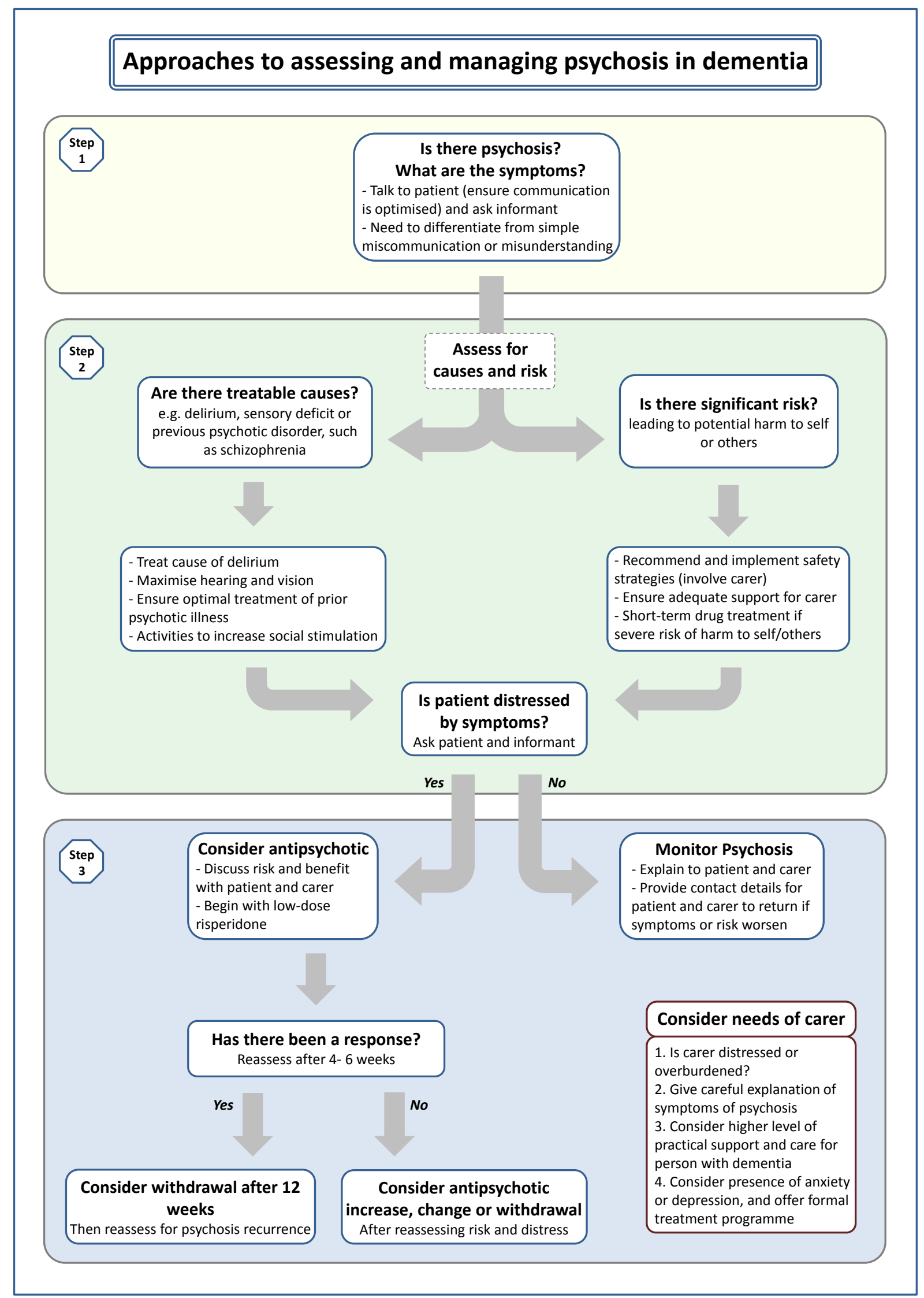

Figure 10. Approaches to assessing and managing psychosis in dementia (see text for further details) 


\section{Indications for using antipsychotics in people with dementia}

Antipsychotic medication should only be used when symptoms cause distress or increased risk; for example beliefs that someone is trying to harm the patient or poisoning their food. A discussion with the patient, their family and staff to decide whether possible benefits are likely to outweigh risks should be documented. Medications should be used to "treat to target": if they do not improve the target symptom, they should be reassessed and either titrated upwards, changed or stopped altogether. There is limited evidence for the efficacy of antipsychotics in treating psychosis in dementia; this is mainly for risperidone $0.5-1 \mathrm{mg}$, the only antipsychotic specifically licensed for use in dementia in US, Europe and UK; with some evidence for aripiprazole (376;389). For other antipsychotics, lack of evidence of efficacy is not necessarily evidence of lack of efficacy but pooled study data suggests that quetiapine and olanzapine are not effective (389;402-404).

\section{Withdrawal of antipsychotics}

Even when antipsychotics are effective, treatment discontinuation should be considered after up to 12 weeks. One double- blind RCT of antipsychotic discontinuation found that for most people with $A D$ who have been on antipsychotics for prolonged periods, withdrawal had no detrimental effect on cognition or functional status, but those with the most severe neuropsychiatric symptoms may have benefited from continuing on antipsychotics (405). In patients with dementia and psychosis with agitation, who had taken antipsychotics for 32 weeks, discontinuation caused more relapses $(60 \%$ on placebo vs $33 \%$ remaining on risperidone) (406), and this is supported by other studies (407). Withdrawal of antipsychotics should be considered for all but with caution for those who had associated agitation and distress.

\section{Key points and recommendations}

New onset psychosis may be due to treatable causes such as delirium or related to hearing loss and other sensory deprivation. These should be considered and, if present treated. Many patients with psychosis in dementia are not distressed and do not need antipsychotics or other drug treatment. A minority of patients who are very distressed or are at risk to themselves or others may benefit from medication in addition to psychological, environmental and social approaches.

There is some evidence to support the use of antipsychotic drugs, particularly risperidone $0.5-1 \mathrm{mg}$, in severe psychosis in dementia, but these drugs lead to an increased risk of serious adverse outcomes which should be discussed with the patient and family. These should be reviewed and withdrawal considered after 12 weeks.

Medications should "treat to target".

Rivastigmine and donepezil may be helpful in hallucinations in DLB.

\section{Agitation}

Many people with dementia show a range of behaviours including restlessness, pacing, repetitive vocalisations and verbally or physically aggressive behaviour that is usually described as agitation $(408 ; 409)$. The behaviours are often accompanied by a feeling of inner tension, although this is more difficult to detect in people with more severe dementia. The cause of these symptoms varies. They 
may be a communication of physical or psychological distress, a misinterpretation of threat, or result from delusions or hallucinations, in a person with dementia-related brain pathology that reduces abilities to communicate, satisfy or even know their needs, and makes it more likely they will repeat a behaviour (409-411). Agitation is often most prominent or problematic during personal care. Aggressive behaviours are usually conceptualised as a subtype of agitation, as in the CohenMansfield Agitation Inventory, although not in the neuropsychiatric inventory (NPI) (409). In many studies and in the NPI agitation subscale, a person with agitation (or aggression) is described as being uncooperative or difficult to handle (324).

Agitated behaviours are common in dementia, more so in moderate or severe dementia, with around half of people with dementia exhibiting such behaviour occasionally every month, and over $20 \%$ having clinically-significant symptoms (377). The rates vary depending on the setting, but are more common in care homes, possibly partly because the symptoms are associated with the breakdown of care in domestic settings and care home admission. The symptoms are persistent (374), so that nearly $40 \%$ of those with significant agitation still had symptoms six months later (377) and $60 \%$ of those with aberrant motor behaviour on the NPI, such as pacing or doing things repetitively, remained symptomatic 18 months later (373). Caring for an agitated person with dementia is more difficult and time consuming that caring for those without agitation; the additional costs of managing agitation account for around $12 \%$ of the costs of dementia (412).

\section{Assessing and managing agitation in dementia}

Figure 11 outlines approaches to managing agitation in dementia. This should start with asking the person what is wrong. If they cannot say, important causes of agitation to be considered and addressed include the person feeling frightened; hungry, thirsty, hot or cold. People who suddenly become agitated may be physically unwell, in pain or delirious. Carers should be consulted about the likely causes of the behaviour, including triggers, and unmet needs. Carers' reactions to agitation may relieve or increase it. Overstimulating or complex environments may also exacerbate agitation. 


\section{Personal care}

Communication skills and person-centred care are about considering what the person with dementia understands, is trying to say and what they want, rather than being focused on completing a task, like personal care, to help them. It involves verbal and non-verbal communication.

This example is about being aware that someone with dementia who requires personal care may not understand or remember this. When that happens, well-intentioned, necessary care may be experienced as assault and the person may become agitated. The principles of communication are to:

- Identify yourself and others if patient does not remember.

- Explain what is happening, when it is happening, one step at a time (as the person with dementia may not remember).

- Use calm, reassuring tones.

- Ensure you can be heard.

- Avoid negative words and tone.

- Ask one thing at a time.

- Speak slowly.

- Allow the person with dementia sufficient time to respond.

- Offer simple choices (no more than two at a time).

- Help patient find words for self-expression (and check if you have understood correctly).

- Lightly touch to reassure, calm, or redirect.

- Use relaxing sensory stimuli, such as music or soft lighting, if they enjoy it.

Box 3. Example of communication skills and person-centred care for agitation during personal care

\section{Treating agitation in dementia}

\section{Interventions to improve communication as treatments for agitation}

A recent systematic review of RCTs calculated standardised effect sizes (SES) of psychological and social interventions for agitation immediately and in the longer term (figure 13) (413). Interventions focused on staff in care homes improving communication with residents with dementia and identifying their wishes (called person-centred care, communication skills training or adapted dementia care mapping), decreased symptomatic and severe agitation immediately (SES 0.3 to 1.8; $\mathrm{SES}=1.4$ ) and up to six months afterwards (SES $=0.2$ to $2.2 ; \mathrm{SES}=1.5$ ). Box 3 exemplifies use of communication skills to decrease agitation. 


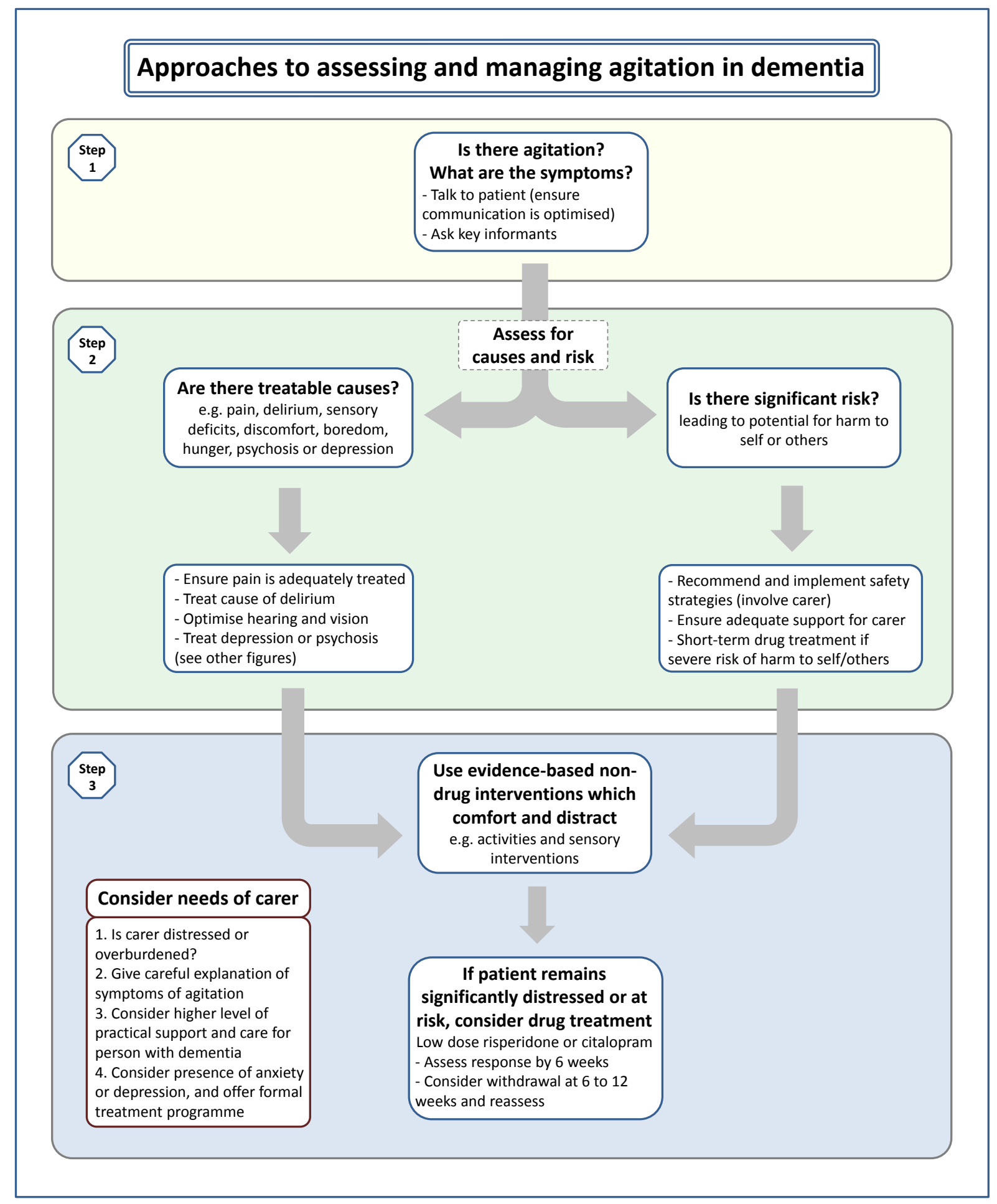

Figure 11. Approaches to assessing and managing agitation in dementia (see text for further details)

\section{Pleasant activities and occupational interventions for agitation}

Most people enjoy activities that interest them and become restless when bored. Engaging in meaningful and pleasurable activities is hypothesised to improve health and wellbeing by reconnecting individuals to their physical and social environment; support self-esteem; build neural 
connections through complex interactions; and promote a sense of role continuity, purpose or personhood, self-identity and meaning (169).

Activity can be a therapeutic agent to target agitation in individuals with dementia at home (414), in hospitals $(415)$ or in residential settings $(413 ; 416)$, while they are engaged in it. One systematic review found that activities in care homes reduced participants' levels of agitation significantly during the activity ( $\mathrm{SES}=0.2$ to 1.05 ) as did music therapy using a protocol ( $\mathrm{SES}=0.5$ to 0.6 ) (figure 13) $(413 ; 414)$. It was unclear whether individualising activities further reduced agitation, perhaps because the activity was effectively individualised as those able and interested engaged in it. There was no evidence effects lasted beyond the intervention period, or for benefit in severe agitation (417). As activity reduces supervision time, it may be cost-effective (415).

As cognition deteriorates, the types of activities people like and can do, and the frequency and level of participation they can manage change (168), as ability to initiate, plan, and organise activities deteriorates. Figure 12 summarises strategies for individualising activities and pleasant events for individuals with varying cognitive levels for therapeutic use $(418 ; 419)(169 ; 420)$. 


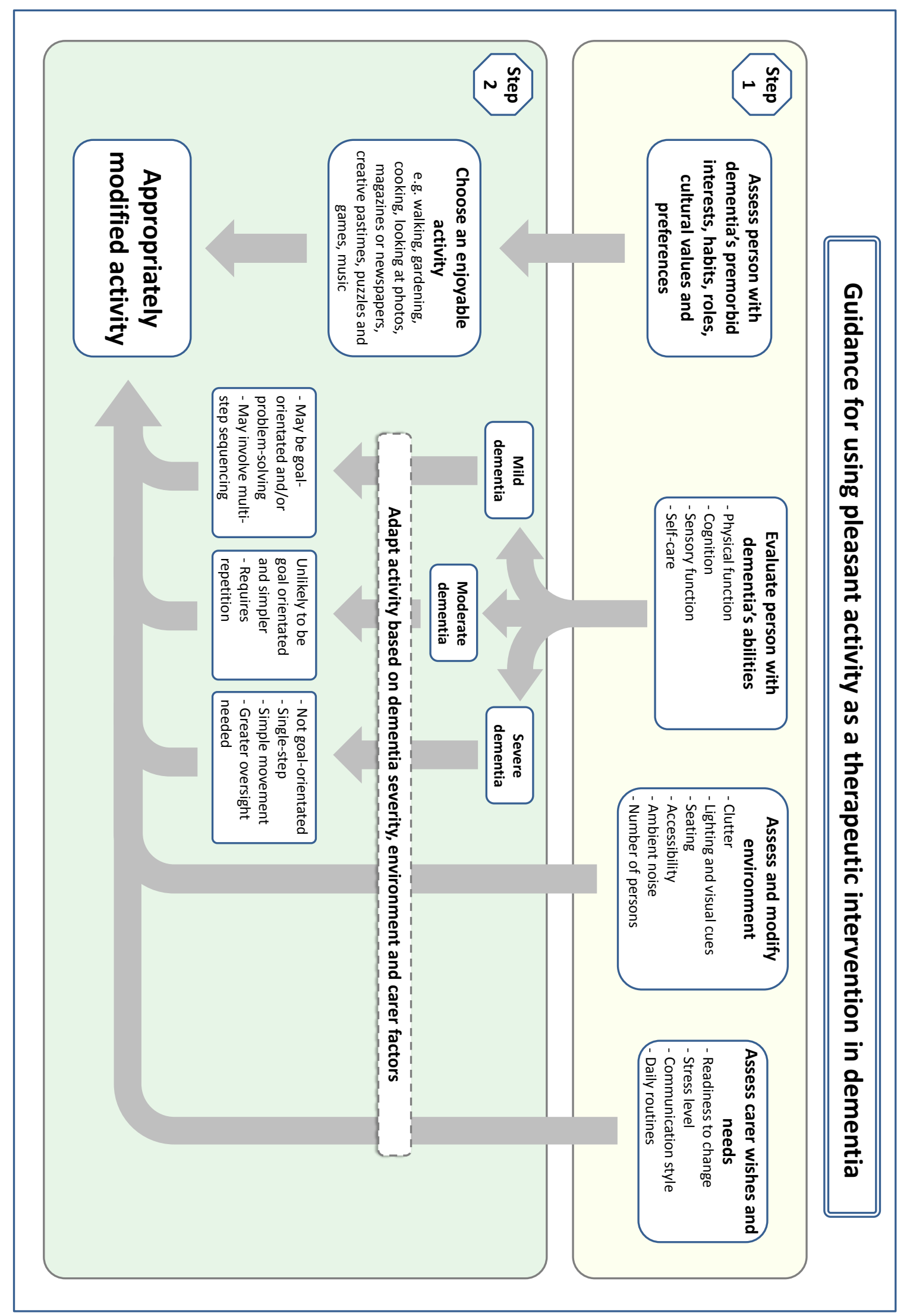

Figure 12. Guidance for using pleasant activity as a therapeutic intervention in dementia

Figure inspired by Regier et al (421) 


\section{Social engagement and sensory interventions for agitation}

Social engagement is a necessary condition for wellbeing throughout life, and the lack of it may cause agitation in people with dementia. It encompasses physical proximity to others, eye contact, conversation and sensory stimulation including touch. It is suggested that social activity may improve quality of life among people with dementia although this has not been shown in high quality RCTs (422).

A systematic review found that clinically-significant agitation reduced during sensory interventions, including massage (413). For many successful group interventions, positive social engagement may be an important mechanism.

In care homes, personal care is an opportunity for positive one-to-one social interactions, but in practice communication is often minimal or comprised of commands or instructions (423). Training staff how to communicate with people with dementia during personal care may be useful. In the U.K., the ongoing Managing Agitation and Raising QUality of lifE in dementia study (MARQUE) is quantifying the frequency of agitation in care home settings and determining the efficacy of a manualised approach to training care home staff to improve every day communication and interaction with people with dementia (424).

A before-after intervention study in 111 nursing home residents with severe dementia found live social stimuli (e.g., with people) decreased agitation more than activities (e.g. folding envelopes; reading; music) (425). Similarly, one-on-one social interaction, music, and watching a videotape reduced agitation (426). Live social stimuli (visit from a baby or a pet, and one-to-one social interactions) also increased pleasure more than exposure to a life-like doll or robotic animal which may be an activity rather than a simulated presence (427). Another open study offering social interaction, environmental modification or personalised music, found that social interaction was most often effective (428). An open study providing different social stimuli for people with dementia in care homes found that residents spent more time interacting with humans than animals and with animals as opposed to toys (419).

Reviews of studies of simulated presence therapy using audiotapes of families found inconclusive evidence of efficacy in any domain (239;429). Unpleasant stimuli, that are experienced as an invasion of personal space or threat may cause agitation (430).

\section{Other non-pharmacological interventions for agitation}

Aromatherapy and light therapy have not been found to be effective for agitation (figure 13) (413). There is no evidence from RCTs that exercise reduces agitation in care home residents (413). 


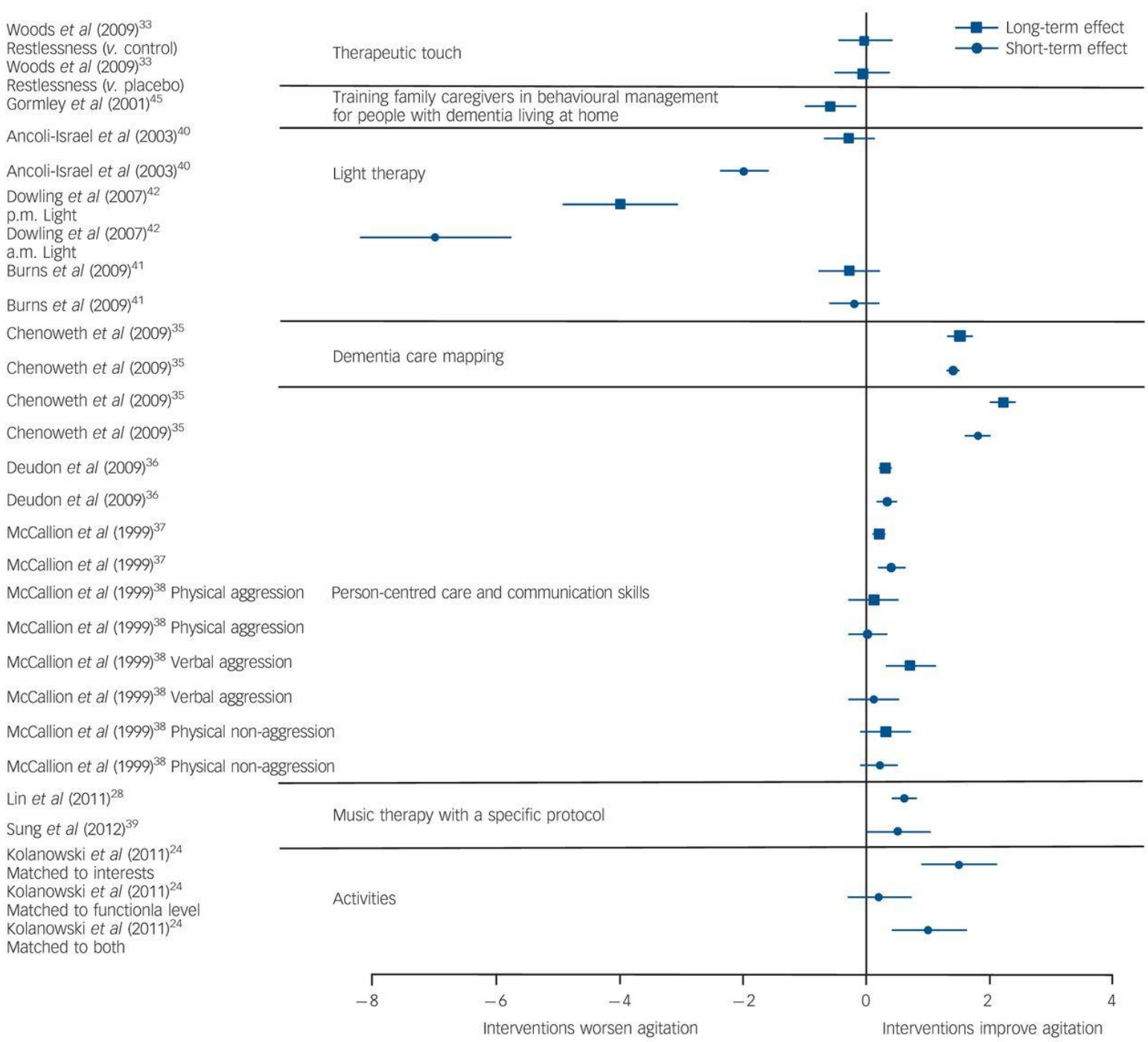

Figure 13. RCTs of effect of psychosocial interventions vs controls for agitation in dementia; standardised effect size and $\mathbf{9 5 \%}$ confidence intervals, where calculable, for agitation immediately and in the longer term

Figure reproduced from Livingston et al (413) by permission of The Royal College of Psychiatrists

\section{Drug treatment of agitation}

\section{Antipsychotics for agitation}

Antipsychotics were the first choice drugs for agitation in dementia, until evidence of their harmfulness demonstrated the need for cautious prescribing and monitoring. Antipsychotics have limited efficacy for aggression; risperidone at a modal daily dose of $<1 \mathrm{mg}$ improved agitation and psychotic symptoms, particularly where aggression was the target symptom; possibly more in severe aggression, with a difference of around 1-1.5 points on the Cohen Mansfield Agitation Inventory (CMAI) subscale when compared to placebo (431). Haloperidol also has effects on aggression though not on other symptoms of agitation. Olanzapine, and quetiapine did not improve psychosis, aggression, or agitation but aripiprazole may improve agitation (431). Overall risperidone has the best evidence for benefit of any atypical antipsychotic, but only over 12 weeks (431). Withdrawal 
trials of antipsychotics do not find an effect on agitation or neuropsychiatric symptoms except for those who have most severe symptoms $(405 ; 432)$.

\section{Other drugs for agitation}

Drugs for cognition, including donepezil and memantine have not been shown to be useful for agitation in RCTs and agitation can be an adverse effect of ChEls (433;434). A double-blind RCT of memantine withdrawal suggested no advantage in the treatment of neuropsychiatric symptoms including agitation (435).

Citalopram showed efficacy for agitation with a 0.93 point difference on the Neurobehavioral Rating Scale agitation subscale (NBRS-A) and clinical global rating (the co-primary outcome) and a 2.4 point difference in the total CMAI compared to placebo, although it causes QT prolongation (436) and worsening of cognition (437). Notably about half of patients responded later in the course of the 9 week clinical trials (438). Pharmacokinetic studies suggested that (R)-citalopram enantiomer, more than the (S) enantiomer accounted for a significant proportion of the adverse effects and deteriorating cognition, as well as less likely treatment response (439) and this may be a future avenue. Like other SSRIs, citalopram can cause akathisia and other extra-pyramidal symptoms (440), although they do so less commonly than antipsychotics. An analysis to assess heterogeneity of response showed that it was not effective for those with more severe agitation, with more impaired cognition and in patients who resided in long-term care (441). It showed no efficacy on the agitation scale of the neuropsychiatric inventory (442). The dose used was $30 \mathrm{mg}$ and the maximum dose usually used for people over 60 or 65 in both the UK and FDA labelling is $20 \mathrm{mg}(443)$

Citalopram was compared to antipsychotics in two earlier trials for behavioural symptoms, including agitation and psychosis in hospitalised non-depressed patients with dementia, where it was no less efficacious than the antipsychotic but both showed low tolerability with more than half of participants dropping out due to illnesses, side-effects and lack of efficacy. In one trial, citalopram (mean dose of $31.1 \mathrm{mg}$ ) was prescribed at a higher dose than now recommended and risperidone was given at a mean dose of $1.36 \mathrm{mg}$; drop outs were very high for both drugs at $44 \%$ for each over the 12-week trial, but there were fewer adverse events with citalopram (444). In the second trial, citalopram $20 \mathrm{mg}$ was more effective than placebo for agitation for up to 17 days; discontinuation rates for citalopram, perphenazine $6.5 \mathrm{mg}$ and placebo were all over $50 \%$ for all three groups (445).

A recent pilot $\mathrm{RCT}$ of dextromethorphan/quinidine suggested benefit in the treatment of agitation with good tolerability (446) and further RCTs are underway.

A non-placebo-controlled trial of stepwise increase in analgesia over 8 weeks for residents with moderate-to-severe dementia and behavioural disturbances in a nursing home found a 7 point difference in the CMAI and a decrease in general neuropsychiatric symptoms four weeks after the end of the study (447) although the reduction of 13 points in the CMAl in the placebo arm of another trial in care homes (433) suggests cautious interpretation. Preliminary evidence has suggested effects of carbamazepine and mirtazapine, which are currently being trialled in the UK.

\section{Key points and recommendations}

Agitation may be due to discomfort, physical illness, delirium or pain which require treatment.

Carer response and overstimulating environment may also worsen agitation. 
There is a human need for social contact and this includes people with dementia. Families and care staff often need help in the skills of maintaining communication and social contact. Interventions to improve communication, activities and sensory interventions are first-line therapy after physical comfort is established. Activities can effectively engage people with dementia, and be integrated within diverse settings. They can help agitation in care homes while they are happening.

Psychotropic drugs for agitation should be used only when there is a high risk or other strategies are unsuccessful and patients are significantly distressed. Antipsychotics are of limited efficacy in agitation in dementia but risperidone 0.5 to $1 \mathrm{mg}$ daily may be used for severe aggression, to prevent harm to the patient or others. Citalopram also might benefit agitation - especially in those with milder $A D$ and milder agitation - but has important side effects (which are different and often less than those of antipsychotics). Adverse events include prolonged QT interval, cognitive impairment, falls, hyponatremia, akathisia and other extra-pyramidal symptoms (448).

\section{Depression}

Depression is common in dementia. Estimates of its prevalence vary but probably more than $20 \%$ of people with dementia have diagnosable depression at any one time, and many others have some depressive symptoms (449). It is distressing, reduces quality of life, exacerbates cognitive and functional impairment, and is associated with increased mortality and carer stress and depression $(450 ; 451)$. Many people with mild depression improve without specific treatment although the services they see are likely to address, at least in part, situational factors predisposing to depression, such as loneliness, under-stimulation from lack of activity or a depressed carer, (452). Those with depression in dementia are likely to have a different neurochemistry compared with those who have had depression without dementia and this might partly explain the poorer response to antidepressant treatment $(453 ; 454)$

Evidence for treatment of depression in dementia is heterogenous. While somewhat speculative, it is likely that depression in dementia differs from depression in those without dementia in biological, psychological and social terms $(452 ; 455)$. One suggested classification of depressive features in dementia includes: (1) a group where depression is situationally determined as a reaction to the impacts of dementia; (2) a homophenotypic group where the syndrome looks like depression but may differ biologically and be related to neurodegeneration and (3) a group with a past history of depression (which is a recurrent disorder) or who develop a 'true' episode of major depressive disorder in dementia. While we do not know from trial evidence, it is likely that a previous good antidepressant response will predict future response.

\section{Principles of assessing and managing depression in dementia}

Figure 14 summarises the approach to assessing and managing people with dementia who have depressive symptoms. It is important to consider whether they are at significant risk, particularly of harming themselves intentionally or by self-neglect, and address these using strategies, possibly including hospital admission if at serious risk. Hypoactive-type delirium or pain may present with depressive features so these should be considered and if present treated. Careful assessment is required to differentiate the features which can be part of dementia, such as apathy, poor concentration or memory; from a depressive disorder and delineate the severity of depression. 
Treatment should be tailored to the patient's needs and wishes and depend on the depression's severity, as detailed below.

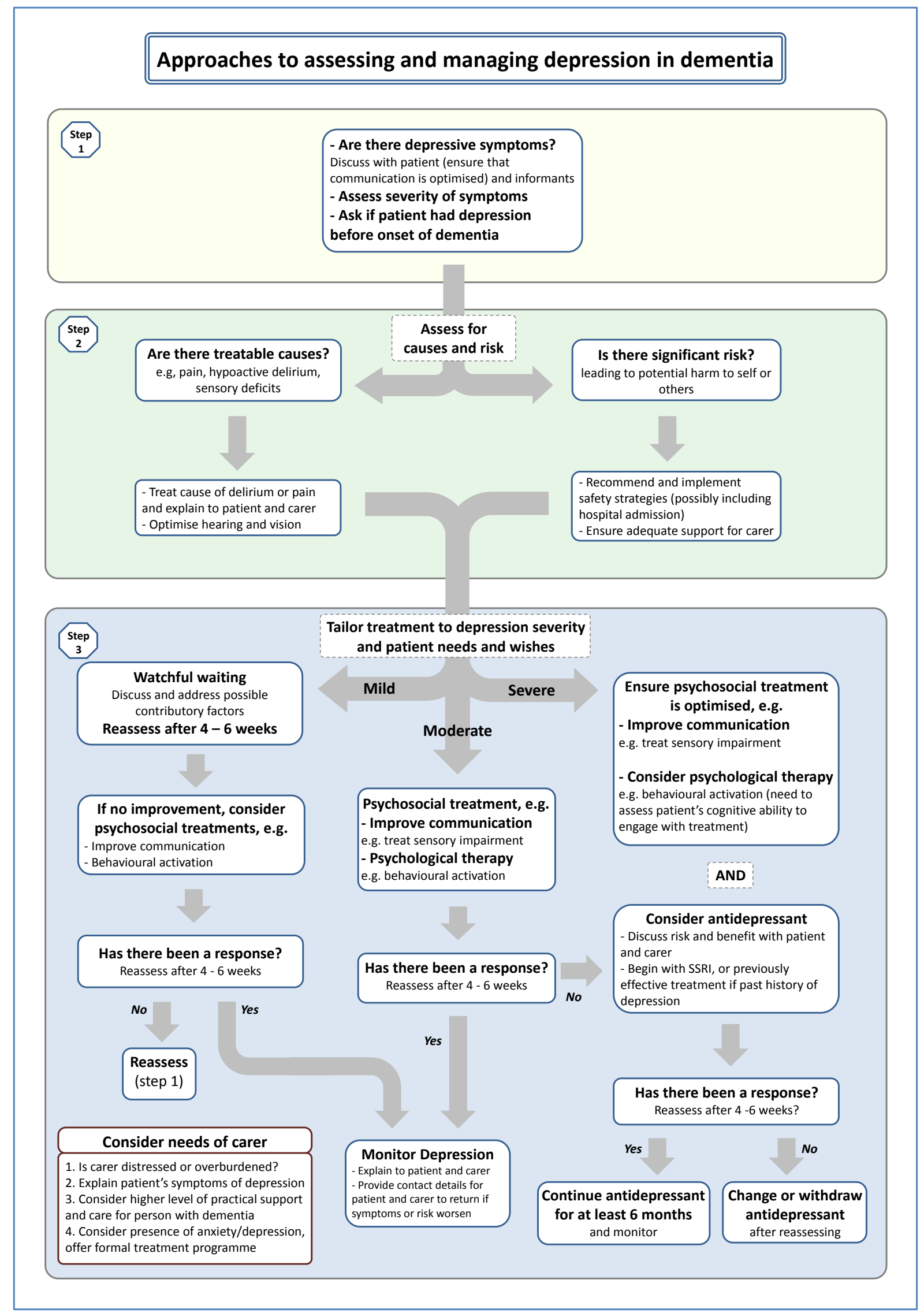


Figure 14. Approaches to assessing and managing depression in dementia (see text for further details)

\section{Treating depression in dementia}

Psychological therapy including cognitive therapy and behavioural activation for depression There is inconclusive evidence that psychological therapies may have an effect in treating symptoms of depression in people with dementia. A recent systematic review and meta-analysis identified six RCTs of psychological therapies involving 439 participants with dementia and depression or depressive symptoms. Overall, psychological therapies including CBT, interpersonal therapy or counselling, compared with treatment as usual, were effective in slightly reducing depressive symptoms (SMD $=-0.22,95 \% \mathrm{Cl}[-0.41$ to -0.03$]$ ) but there the quality of the evidence was limited(456). Only one of the individual studies was effective (figure 15) (457). Psychological treatment reduced clinician-rated anxiety measured with the Rating Anxiety in Dementia scale (mean difference $=-4.57,95 \% \mathrm{Cl}[-7.81$ to -1.32$]$ ), but not self-rated or carer-rated anxiety (457), although this evidence was also of low quality. There is also preliminary pilot study evidence that behavioural activation including pleasant events and engaging in activities may reduce depression (171).

\begin{tabular}{|c|c|c|c|c|c|c|c|c|c|}
\hline \multirow[b]{2}{*}{ Study or Subgroup } & \multicolumn{3}{|c|}{ Experimental } & \multicolumn{3}{|c|}{ Control } & \multicolumn{2}{|c|}{ Std. Mean Difference } & \multirow{2}{*}{$\begin{array}{l}\text { Std. Mean Difference } \\
\text { IV, Fixed, } 95 \% \mathrm{Cl}\end{array}$} \\
\hline & Mean & SD & Total & Mean & SD & Total & Weight & IV, Fixed, $95 \% \mathrm{Cl}$ & \\
\hline Burgener 2008 & 3.3 & 2.9 & 19 & 4.3 & 3.4 & 14 & $7.4 \%$ & $-0.31[-1.01,0.38]$ & \\
\hline Burns 2005 & 5.4 & 2.6 & 20 & 5.5 & 3.1 & 20 & $9.3 \%$ & $-0.03[-0.65,0.59]$ & \\
\hline Spector 2012 & 10.38 & 5.835 & 21 & 16.72 & 7.283 & 18 & $8.0 \%$ & $-0.95[-1.62,-0.28]$ & \\
\hline Stanley 2012 & 8.2 & 2.86 & 11 & 7.8 & 5.95 & 15 & $5.9 \%$ & $0.08[-0.70,0.86]$ & \\
\hline Tappen 2009 & 15.13 & 9.54 & 15 & 19.13 & 7.37 & 15 & $6.8 \%$ & $-0.46[-1.18,0.27]$ & \\
\hline Waldorff 2012 & 5.05 & 4.61 & 130 & 5.77 & 5.07 & 141 & $62.7 \%$ & $-0.15[-0.39,0.09]$ & 十 \\
\hline Total $(95 \% \mathrm{Cl})$ & & & 216 & & & 223 & $100.0 \%$ & $-0.22[-0.41,-0.03]$ & \\
\hline \multicolumn{9}{|c|}{$\begin{array}{l}\text { Heterogeneity: } \mathrm{Chi}^{2}=6.33, \mathrm{df}=5(\mathrm{P}=0.28) ; \mathrm{I}^{2}=21 \% \\
\text { Test for overall effect: } Z=2.30(P=0.02)\end{array}$} & 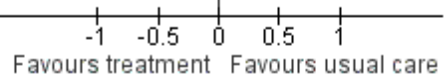 \\
\hline
\end{tabular}

Figure 15. Effect of psychological treatment vs treatment as usual; outcome is depression

Figure reproduced from Orgeta et al (456) by permission of the Cochrane Database of Systematic Reviews

\section{Exercise for depressive symptoms}

A recent Cochrane review found no significant benefit from exercise on depression (SMD 0.14, 95\% $\mathrm{CI}[-0.07$ to 0.36]) (336). However the RDAD (Reducing Disabilities in Alzheimer's Disease), based on the Seattle protocols (414), included exercise training, carer education and problem-solving to enable and encourage participation in enjoyable exercise and found that the combination improved physical disability in 153 people with $A D$ and there was a small (possibly not clinically-significant) difference in depressive symptoms but exercise may not have been the active component (304). 


\section{Drug treatments for depression}

Antidepressants are often the first-line therapeutic option for depression in dementia; but lack definitive evidence for their effectiveness (458). Despite this, people with $A D$ are three times as likely to be prescribed antidepressants as those of the same age without dementia (459).

The Cochrane review of antidepressants for treating depression in dementia concluded that the evidence for clinical-effectiveness was equivocal and weak (460) and that the small possibility of positive effect was driven by the preliminary DIADS study of sertraline which was highly positive (461). Since that review, the much larger DIADS-II $(n=131)(462 ; 463)$ and HTA-SADD $(n=326)(452)$ studies did not find that sertraline was superior to placebo in the treatment of depression in dementia. Although most people included did not have severe depression, there was no indication of a difference according to the severity of depression. Few trials have investigated the effects of newer, non-SSRI antidepressants on depression in dementia but the HTA-SADD trial found that mirtazapine, a noradrenergic and specific serotonergic antidepressant, was also no better than placebo treatment over 13 and 39 weeks. There are a few older and generally smaller trials which have investigated tricyclic antidepressants and monoamine oxidase inhibitors (460). Although an earlier study recruited 694 patients to compare moclobemide $400 \mathrm{mg}$ to placebo; only 511 participants had dementia (all types), it did not use outcome measures validated in dementia, and it is not possible to separate the data of those with dementia from the rest of the participants who had cognitive decline (464). Like this study, others often do not meet the quality thresholds for inclusion in systematic reviews and the outcome measures used are not optimised for dementia (454). The lack of efficacy in treating mild-to-moderate depression with antidepressants or psychological interventions is perhaps understandable as we are trying to treat a complex, heterogeneous, multifactorial phenomenon with a simple intervention. Most studies that have evaluated the effectiveness of antidepressants in people with dementia exclude people with severe depression.

There are very few data on the response to antidepressants in people who have a pre-existing history of depression, predating the first symptoms of dementia may be similar to that in depression without dementia. As discussed above, depression may be a prodromal symptom of dementia but can also occur in those who have a long past history of depressive disorder. However it is plausible that there might be attenuation in the treatment response due to the neurodegeneration and neurochemical changes that are part of dementia. Although we do not have trials in this specific group, it seems unlikely that dementia would make them entirely resistant to previously effective psychological or drug therapy. In the absence of trial data, clinical practice for those who have a past history of treatment response to antidepressants prior to their developing dementia would be to use this treatment as a first-line treatment for depressive episodes following the diagnosis of dementia.

Overall, despite being very commonly used, the evidence for antidepressants having a positive role in dementia is weak. There is also no good evidence that antidepressants are effective in improving other outcomes, such as ADL, cognition, clinical severity, or carer burden. There are however harms that are attributable to antidepressants, which are common and sometimes serious (discussed in agitation section, above) $(436 ; 437 ; 452 ; 462)$. In view of these adverse effects and the lack of evidence for positive effects, they should not be used in those without a history of depression in younger age, unless psychosocial treatments are unsuccessful. Some individuals may benefit from antidepressants but we do not have trial data with which to identify this group. Clinical decision making will as always rely on an individualised assessment of risks, harms and potential benefits. The 
dilemma of using antidepressants in dementia is highlighted by the apparent paradox that once started, they may be difficult to stop and it is unclear how long they should be continued. The one RCT of antidepressant discontinuation was in nursing home residents with dementia and found that discontinuation led to increased depressive symptoms (465). While this suggests efficacy in this group, it may also be that the increase in depressive symptoms is a transient withdrawal syndrome. There are no similar studies in community settings or those with a less severe dementia.

\section{Key points and recommendations}

Many people with dementia and depression will improve with time. Management of possible contributory factors to depression should be encouraged. There is inconclusive evidence that increasing activity, decreasing isolation and talking therapies may help depressive symptoms and we await definitive trials. In the meantime these should be the first-line management in mild-tomoderate depression in dementia.

Antidepressants have not been shown to be effective in dementia and have side-effects so are not first-line treatments for depression in dementia.

We recommend not starting antidepressants in people with dementia unless there is a history of depressive episodes prior to the dementia or the patient has not responded to social or psychological treatment and is moderately or severely depressed.

Stopping antidepressants in people with severe dementia can lead to increased depressive symptoms.

\section{Sleep}

Sleep disturbances in older people with dementia are aetiologically heterogeneous and complex, occurring in $25-55 \%$ of those with neurodegenerative dementias (466-468). They may be caused by one or more of pain and physical health conditions; anxiety; lack of activity and neurodegenerative changes. Impaired melatonin production occurs in $A D$ and other dementias because of Suprachiasmatic Nucleus neuronal loss $(469 ; 470)$, leading to a decreased regularity of sleep, impaired sleep initiation and continuity, and difficulty maintaining wakefulness during daylight. Sleep disturbance predicts family carer depressive symptoms, increases care burden and leads to care home admission, significantly elevating care costs (471). A Cochrane review found no definitive evidence from trials of pharmacological treatments for sleep in older people with dementia (ChEls, donepezil and galantamine; antidepressants, trazodone and mirtazapine; or melatonin and ramelteon) and there were no RCTs of benzodiazepines or non-benzodiazepine hypnotics (472).There was some suggestion that trazodone $50 \mathrm{mg}$ may be useful but no large trials.

Bright light therapy used in this group, without measuring patients' individual disturbed circadian rhythm has also been ineffective (figure 16) (473). Most evidence about sleep hygiene and light comes from small, often pilot, studies with low methodological rigour, leading to insufficient and conflicting evidence (474). Nevertheless preliminary evidence from a pilot RCT of 36 participants suggests that light therapy, and activity may help sleep, as may education and behavioural techniques (475). Light therapy may come from natural light, a dawn simulation alarm, or light boxes and does not necessarily require the patient to remain still. Actigraphs, which are worn like watches, and measure the patient's activity, light exposure and circadian rhythm, allow for an attempt to anchor circadian rhythms to day and night with light therapy. 
As there are currently no treatments with definitive evidence of effectiveness, health teams use a mixture of sleep hygiene measures and psychotropic medication, extrapolated from other conditions. Patients in nursing homes taking benzodiazepines or Z-drugs (the non-benzodiazepine sedatives, e.g., zopiclone, eszopiclone, zaleplon, zolpidem, or zelmid) had worse sleep at baseline than those who did not, but over a year both groups deteriorated with those taking hypnotics doing no better than those who did not (476). Benzodiazepines also immediately increase the risk of falls (477). Thus without definite benefits, and with strong evidence of harm including increased mortality in general populations of older people (478), z-drugs and benzodiazepines should be avoided, if possible (472).

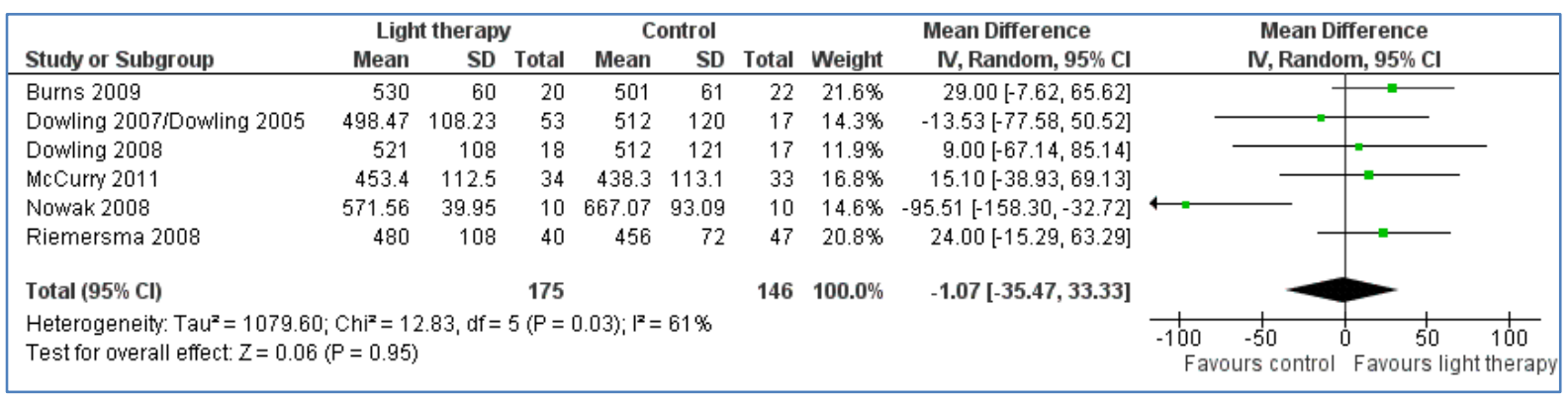

\section{Figure 16. Effect of 10 days to 10 weeks of bright light treatment on total sleep duration}

Figure reproduced from Forbes et al (473) by permission of the Cochrane Database of Systematic Reviews

\section{Rapid eye movement (REM) sleep behaviour disorder}

Rapid eye movement (REM) sleep behaviour disorder occurs commonly, in around $20 \%$ of DLB patients and in Parkinson's disease dementia (468). REM sleep disorder causes vivid, frequently frightening, dreams and loss of sleep paralysis during REM sleep, allowing motor activity or dream enactment, including aggression and fleeing, thus risking injury to the patient or bed-partner $(479 ; 480)$. Practical measures to prevent injury from falling out of bed, for example, a bed rail, can be used and low-dose oral clonazepam ( 0.25 to $2 \mathrm{mg}$ ) can suppress REM sleep. Cohort studies have found that it works well in most people; studies of melatonin in non-responders are very small $(372 ; 480)$.

\section{Key points and recommendations}

Sleep disorders are aetiologically heterogenous and the cause of sleep problems may be pain or discomfort as well as dementia. There are preliminary indications that sleep may respond to a combination of tailored light therapy and sleep hygiene.

There is no definitive evidence that any drug management works in sleep disorders in most dementias and they can harm.

REM sleep behaviour disorder in the Lewy body dementias may respond to low dose clonazepam.

\section{Apathy}

Apathy is one the commonest and most persistent neuropsychiatric symptoms (374). In a review of the largest non-pharmacological intervention studies. Fifteen of seventeen studies of tailored activity and eight of the nine studies using non-tailored activity reported a positive or partly positive 
outcome (481). In the Improving Well-being and Health for People with Dementia (WHELD) study, antipsychotic review combined with social activity or exercise led to a reduction in apathy as a secondary outcome (482). The ADMET (Alzheimer's Disease Methylphenidate Trial) with 60 people given $20 \mathrm{mg}$ methylphenidate or placebo found no difference in the apathy evaluation scale but more people in the intervention group rated as mildly to markedly improved (483) Therefore, although there are no definitive trials on management of apathy, there is an indication that interventions that increase activity or methylphenidate may be helpful. Figure 12 summarises strategies for using activity with people with dementia.

\section{Caring and supporting}

\section{Family carers as decision makers}

Family carers are the most important resource available for people with dementia (233). Caring can bring emotional rewards but also difficulties for a family member. When dementia is mild, decisions about everyday life, social care and medical treatment can usually be made by the person with dementia, usually with support from family or friends. As dementia progresses, the person with dementia loses the mental capacity to make more complex decisions and the carer becomes the substitute decision maker, changing the relationship of partners and reversing the role of parents with children $(236 ; 237 ; 484 ; 485)$. A substitute decision maker's best interest decision includes consideration of what the person would have wanted rather than the decision maker's judgement of beneficence. Figure 17 sets out, as an example, the process of assessing mental capacity within the UK legal framework. There is substantial variability regarding legal issues between countries, and between states in the United States.

Families report that the most difficult decisions to support people with dementia to make or to decide as a proxy are how and when to use health and social services for dementia; whether to agree to potentially distressing medical interventions; whether someone should live at home or in a care home; together with legal matters, including power of attorney and driving; and making plans for the person with dementia if their carer was too ill to continue their caring role (486-488). Driving is frequently contentious and some places, for example, UK and California, require notification of a dementia diagnosis, while others have guidelines about driving and dementia. Notification does not automatically lead to a driving ban.

Lasting, Enduring or Durable Power of Attorney, as it is labelled in different countries, allows a person who understands the decision to nominate a trusted person to be an authorised attorney for future decisions should they be unable to make them themselves. A similar legal mechanism for protecting personal and financial welfare for people with dementia includes guardianship or court of protection orders, which are put in place when someone has lost capacity, and cannot appoint an Attorney. 


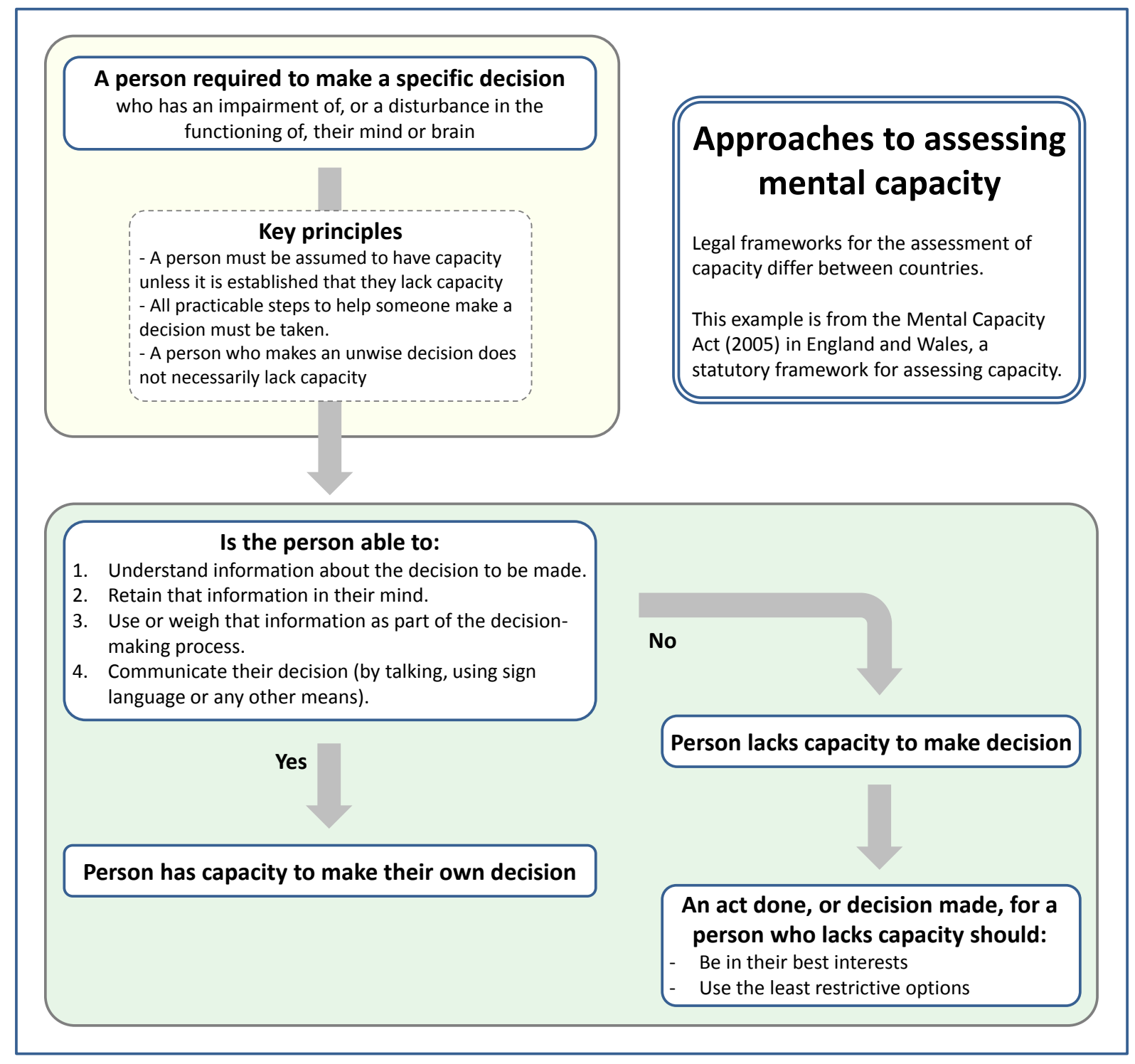

Figure 17. Approaches to assessing mental capacity

In England, the Mental Capacity Act sets out a framework to decide whether someone has the capacity to make a specific decision and, if not, who the designated decision maker is (figure 17). This is most commonly enacted for financial decisions but may be used for decisions on health or social care matters. Most carers welcome the legal authority but still often find it distressing and difficult to make decisions; this is exacerbated by insufficient information, lack of emotional support including family disagreement, being unsure what the person with dementia would have chosen and adhering to a solution conceived before the situation changed $(236 ; 237 ; 486 ; 489)$. Proxy decisionmaking is facilitated by discussions while a person with dementia retains some ability to consider what may happen in future $(486 ; 490)$. Families may require support, immediately after diagnosis and subsequently, and this may usefully be delivered as a professionally supported decision aid. These provide structured information relevant to the decision, which can then be discussed with a knowledgeable facilitator $(488 ; 490)$. Carers who received the DECIDE intervention, a facilitated decision aid to support the decision of whether a person with dementia should move to a care 
home, reduced carer decisional conflict in one small non-blinded RCT (491). Decisional conflict is associated with people not making and regretting decisions.

\section{Key points and recommendations}

Many decisions about health, care and finances are made by the family carer as people with dementia frequently lose mental capacity to make complex decisions. People may be able to contribute to decisions but not make them independently. Capacity is situation specific. Early and ongoing capacity assessment is helpful. Healthcare professionals should discuss how decisions will be made about future care with patients, when dementia is in its early stage, and at any stage with carers.

Using structured decision aids may reduce decisional conflict.

Jurisdiction-specific legal frameworks and guidelines outline processes for assessing decisional capacity, safety to continue driving and appointing a lasting, enduring or durable attorney.

\section{Caring for family carers}

Families usually provide most of the care to people living at home. This can be psychologically and physically demanding. About $40 \%$ of family carers of people with dementia have clinically-significant depression or anxiety; others have important but less severe psychological symptoms (241;492). Family carers have worse physical health, more absences from work and report lower life quality than non-carers (493). Spouses of people with dementia are at increased dementia risk (494). Female, co-resident carers and those looking after someone with neuropsychiatric symptoms are most at risk; although perhaps counterintuitively, caring for someone with more severe cognitive impairment does not predict psychological distress (301;495). Carer depressive and anxiety symptoms impact not only the individual but also their relative with dementia and wider society, as carer psychological morbidity, particularly depression, predicts care breakdown and therefore care home admission (496), and elder abuse (497). Most people like family members with dementia to continue living at home as long as possible and people with dementia have a better quality of life when they do so (498). It is therefore important to know how to effectively prevent or manage such symptoms.

Specialist, individually tailored, multicomponent psychological support to family carers, in which carers make active choices eg the REACH (Resources for Enhancing Alzheimer's Caregiver Health) intervention significantly reduce the rate of, although not necessarily the time to, care home admission (499-501). Some programmes, including those of the Seattle Protocols, have also reported that training family members to understand the interpersonal and environmental aspects of behaviour of relatives with dementia can decrease those problems and relatedly, decrease their own distress. Specialist individual (as opposed to group) behavioural or coping strategy interventions have been efficacious; with six being the minimum number of sessions of individual behaviour management which were needed $(301 ; 502)$. Cognitive behavioural therapy (CBT) and other therapies developed primarily to target depression do not effectively treat carer anxiety (495). Some approaches train carers to identify precipitating events and their role in behavioural difficulties and situation and encourage changing the response or the environmental factors linked these problems, rather than expecting the person with dementia to change (300). The mechanism of these effects may relate to carers changing their coping strategies and using more acceptance-based or emotionfocussed strategies $(503 ; 504)$. 
Education increasing knowledge about dementia is always part of a successful multicomponent intervention but by itself does not seem to improve carers' mental health (505) (506). Similarly, group behavioural therapy, support by trained experienced family carers, support for patient and carer together and two years of education, group reminiscence therapy, counselling and social support were not effective carer interventions (507-509) (510).

One continuing mixed individual intervention for carers was effective by eight months (but not at 4 months) in reducing depression, continued working three years after the intervention started and delayed nursing home placement (511). It consisted of two individual and four extended family sessions (excluding the patient) which encompassed education and strategies around the particular problems, followed by an ongoing support group and the provision of ad hoc counsellors support as needed. It was also successful in reducing care home admission (303). However, six family meetings (two individual and four with the wider family) did not prevent (as opposed to treat) anxiety and depression in the carer (512).

The START intervention (Strategies for Relatives) which was developed from REACH, is a manualbased eight-session therapy, targeted at coping with individual problems but also includes planning for the future and relaxation and left the carer with their own manual with a plan to continue strategies they had found effective (513). It was successful in reducing anxiety and depressive symptoms and both preventing and treating depression in carers and is cost-effective $(242 ; 514 ; 515)$. Its effect continued for 2 years at which point many carers were still using the manual and choosing which of the strategies, including relaxation techniques, they continued to use (516). It is being implemented in the UK and, as it is delivered by supervised psychology graduates rather than highly trained clinical psychologists, it is practical. There is evidence that the REACH intervention programme may generate savings in carer time and therefore in cost but it is expensive as it is delivered by clinical psychologists (517).

\section{Key points and recommendations}

Family carers of people with dementia are at high risk of depression and anxiety disorders. Effective interventions are individually tailored, multicomponent and focus on individual carers (sometimes with their extended family) making active choices. They may work for an extended period and may prolong the time people with dementia can live at home.

Many interventions help carers to understand that they are able to change the situation but the person with dementia usually cannot change themselves. Information by itself is not enough. Many such passive interventions are ineffective so services should use interventions for which there is evidence.

\section{Protecting}

\section{Definitions of abuse}

Abuse is defined as "a violation of an individual's human and civil rights by another person(s)" (518) and can take different forms. These include verbal or psychological abuse, encompassing screaming and shouting, name-calling, threatening or humiliating and physical abuse including hitting, shoving or handling roughly, inappropriate medication use, restraint or confinement. Proportionate selfdefence is not abuse. Neglect (including allowing self-neglect), is defined as ignoring medical or physical care needs, failure to provide access to appropriate health or social care, or withholding the 
necessities of life, such as adequate nutrition, medication, and heating. Financial and sexual abuse involves persuading someone to enter into a financial or sexual transaction to which they have not consented or cannot consent. 'Institutional' abuse encompasses harms arising from institutional policies or routines, for example, only allowing access to food and drink at certain times.

In research, 'cases' of abuse are identified by setting thresholds for the severity or frequency of an abusive behaviour that constitute 'significant' abuse (519). In clinical settings, the terms abuse and neglect are often reserved for serious violations that meet thresholds for formal intervention. Less serious violations, frequently including acts of omission, that meet criteria for abuse are often conceptualised as poor care in clinical practice rather than named as abuse.

Some researchers use the term 'potentially harmful behaviour' in preference to abuse. This may avoid the implication of intent which is often thought to be present in the term abuse, which is pejorative, but fails to distinguish harm that violates human rights from accidental harm. Abuse is sometimes perpetrated consciously but is often behaviour in response to practical management difficulties, without sufficient thought or regard to the violation of human rights it creates (box 4 for case example).

\section{Factors increasing the risk of abuse for people with dementia}

Most people with dementia are not abused, but many older people who are abused have dementia. People may be vulnerable to abuse through isolation, reduced autonomy due to care dependency, controlling relationships with carers or partners and difficulties remembering or communicating their experiences. In the older population, dementia is probably the most common cause of this vulnerability. Over a third of family carers report the person for whom they care behaves abusively towards them (520). Abuse may be reciprocal as people with dementia who are verbally or physically abusive towards carers are especially likely to be abused $(497 ; 520)$.

People with dementia who have neuropsychiatric symptoms, including acting aggressively towards their family carers, and whose family carers feel more burdened, spend more hours caring, and have more psychological morbidity, are more likely to be abused $(497 ; 521)$. That is, unsurprisingly, distressed carers who have more to cope with are more likely to act abusively. Cross-cultural differences reported in the prevalence of abuse in the community probably reflect differences in where people with more severe dementia are cared for, with higher community rates in countries where people with severe dementia are more commonly cared for in their own homes (522), and high rates of abuse in care homes in countries where most people with severe dementia live in this setting (522).

\section{Prevalence of abuse for people with dementia}

Elder abuse is inherently difficult to study. It is hidden, often perpetrated against vulnerable people, by those on whom they depend. Prevalence estimates are influenced, and possibly underestimated, by the inability, fear or embarrassment of older people to report it. Some studies have asked paid or family carers to self-report these behaviours and they appear willing to but may not see as abusive behaviour (519) often arising due to stress and burden. We must measure abuse to develop interventions to reduce it, but care workers reporting abuse face potential adverse legal, 
employment and social consequences, so anonymous reporting is probably necessary for research (523;524), making intervention difficult.

Surveys recording abusive behaviours, without implication of intent, which is generally a legally determined construct, or blame, which is socially determined find that it is more likely to happen to people with dementia. Six percent of older people in the general population reported that they have been subject to significant abuse during the past month; among frail older people, nearly a quarter reported significant levels of psychological abuse. A third of family carers report acting abusively towards people with a diagnosis of dementia living in the community (most with mild or moderate dementia) (519). Sixteen per cent of staff in care homes, where most people have moderate or severe dementia, had witnessed significant psychological abuse (525).

\section{Unintentional abuse}

Problem: Mr Smith moved to a care home when his son, with whom he had lived, moved abroad. Mr Smith continually asked when he would go home and see his son and could not remember his son had moved. Staff avoided Mr Smith because they did not know how to reply. He became increasingly agitated, refused personal care and was sometimes physically aggressive. His skin began to break down through neglect.

Assessment: He was referred to mental health services and a nurse met with staff and talked to his son. Staff discovered that team members had each been responding in different ways - some saying his son was on holiday and he would go home soon, others saying that this was his home now and others not answering him. His son told the nurse that he felt guilty and had avoided calling his father as he thought his calls would disrupt him from settling in the home.

Management: The care staff and nurse worked out that saying his son loved him, and encouraging him to talk about his son helped Mr Smith and agreed they would give that consistent message. They reassured the son that regular contact would help and he started this using skype. Staff worked with family to add personal possessions, and photographs to his room making it more home-like.

Staff also talked to him during personal care, gently explaining what they were doing, and played music that he liked. They planned that staff members he trusted would, when possible, give personal care. He began to accept personal care again.

The staff maintained these strategies when things were better.

\section{Box 4. Case vignette of abuse in dementia and management strategy}

\section{Approaches for preventing and reducing abuse in people with dementia}

Abuse may go unacknowledged if they feel there are no better management options and is underdetected and under-reported (526). Staff who detect abuse may not report it because they do not know how to, or because they empathise with the perpetrator, fear recrimination, or expect responses to be inappropriate and punitive (527). Encouraging naming and reporting of abusive behaviour is an important first step to reducing it. There is good evidence that interventions can effectively increase professionals' knowledge about abuse and their ability to detect and manage it (528;529). 
Managing the most serious cases of abuse, including financial abuse, physical violence and occasionally murder, involves criminal justice systems. National legal frameworks for managing abuse vary; in California, medical professionals have been criminally charged and sentenced under elder abuse laws for the illegal chemical restraint (medication for the sole purpose of sedation) of patients.

Most clinical studies seeking to reduce abusive behaviour, target physical restraints, in care home or hospital settings and often show this is possible using person centred approaches (530). Restraints are defined as anything restricting movement, such as bilateral bed rails, belts, and fixed tables in a chair. They can cause distress, violate human rights, impair future mobility and skin integrity and usually do not prevent falls. Restraints may sometimes be because of society's unwillingness to provide adequate dementia care resources. Care workers delivering care with inadequate training and resources may use restraints to try to prevent harm. The judgement of what is restraint may be granular. Bed rails may be used only to prevent someone with excessive movement during sleep falling out of bed. It might be neglectful abuse not to use them. One carer briefly and gently holding a person's hand during personal care so they do not hit another carer is proportionate and may be comforting. Reduction in physical restraint is an observable outcome and, in countries where physical restraint is permissible in some circumstances, less likely to be hidden.

Any disproportionate restraint is unacceptable, ethical and legal opinions vary about the relative harms of using sedating medication or physical restraint to manage symptoms that may cause harm. Psychotropic medication to manage agitation and aggression would generally be considered more acceptable. By contrast, the Netherlands has traditionally prefered seclusion and physical restraint in preference to medication, although this is changing (531). In the UK and US, there have been well publicised cases of relatives placing cameras in care homes and witnessing abuse. Using monitoring technology to detect harm to people with more severe dementia is one way of detecting abuse to stop it. However, it may compromise a person's privacy and like other interventions, risk and benefits need to be balanced, ideally undertaken with the individual's permission or, if not possible, in their best interest.

There are few examples of intervention studies including elder abuse as an outcome aside from restraint. This may reflect concerns about the validity of asking perpetrators or vulnerable people to self-report abuse, but elder abuse can be measured reliably and with validity (525). In the only intervention study to measure abusive behaviour by family carers as an outcome, found no evidence that the START (STrAtegies for RelaTives) intervention reduced abusive behaviour (514;532). For ethical reasons researchers intervened to manage abuse in both groups, which may have masked any intervention effect. Interventions that aim to reduce burden of care, carer distress and neuropsychiatric symptoms in people with dementia may prevent abuse in community settings but currently there is no evidence demonstrating this. More work to develop definitive interventions to reduce other forms of abuse is needed, including trials with abusive behaviour as an outcome. These should adequately measure and address neglect, which is common. Abuse of older, vulnerable people in society, like child abuse, cannot just be allowed to continue.

\section{Key points and recommendations}

One in four vulnerable older people may experience abuse and only a small proportion is reported. Many older people who experience abuse have dementia. Most abusive behaviour happens where 
quality of care is poor and carers, family or professionals do not have other strategies to manage difficult situations. Abuse is sometimes, but rarely, sadistic.

There is good evidence that person-centred care reduces restraint use in care homes and hospitals and should be implemented.

Identifying abusive behaviour accurately is a prerequisite of testing interventions to reduce it; for paid carers this probably needs anonymous reporting. We can measure abuse in a reliable and valid way. Interventions to increase professionals' knowledge about, ability to detect and manage abuse are needed.

\section{Dying}

Dementia shortens survival, even after controlling for age and multi-morbidity. This varies between populations and progression may be faster in woman participants and those with younger onset dementia (533). A UK population study found a median survival time from diagnosis of dementia to death of 4.1 years (534). In a primary care study, where diagnosis sometimes occurs at a late stage, median survival times from diagnosis were 6.7 years in those diagnosed at age 60-69, falling to 1.9 years for those diagnosed at age $\geq 90$ years (246). Dementia was the sixth leading cause of death in the US in 2011 and 600,000 Americans with AD died in 2014 (535). Given its increasing prevalence it is predicted that one in three people over 60 years old will die with dementia (18).

\section{Defining optimal end-of-life care}

Despite this, dementia is often not perceived to be life-limiting or terminal and there is sometimes a failure to adopt a palliative approach to care (536-538). This may result in poor management of symptoms towards the end-of-life, causing considerable distress to the person with dementia and their family.

Specific difficulties in caring for someone with dementia at the end-of-life include:

A person with dementia may lose cognitive abilities, function, and capacity, in contrast to cancer and other advanced chronic diseases. They may be unable to make decisions about their care and treatment or express their needs and wishes as death approaches.

There is considerable prognostic uncertainty; the course of dementia is unpredictable and varies greatly between individuals. Prognostic tools have been developed but there is little evidence to suggest that knowing the prognosis changes management, improves outcomes such as comfort or is helpful to the person with dementia and their families and carers (539).

It has been argued that we should acknowledge and hold the uncertainty and focus on maximising comfort and quality of life, rather than estimating prognosis (540) or developing strict criteria for when the person with dementia should be able to access hospice care (541). This is in keeping with the goals of palliative care(542):

"The active, total care of patients whose disease is not responsive to curative treatment. Control of pain, of other symptoms, and of psychological, social and spiritual problems is paramount. The goal of palliative care is achievement of the best quality of life for patients and their families." 
The European Association of Palliative Care has defined optimal palliative care for people with dementia (543). In this consensus process, recommendations were made about person-centred care, communication and shared decision-making, optimal treatment of symptoms and providing comfort, setting care goals and advance planning, continuity of care, psychosocial and spiritual support, education of the healthcare team and societal and ethical issues. Their model of care stresses the importance of changing care goals throughout the course of dementia (figure 18).

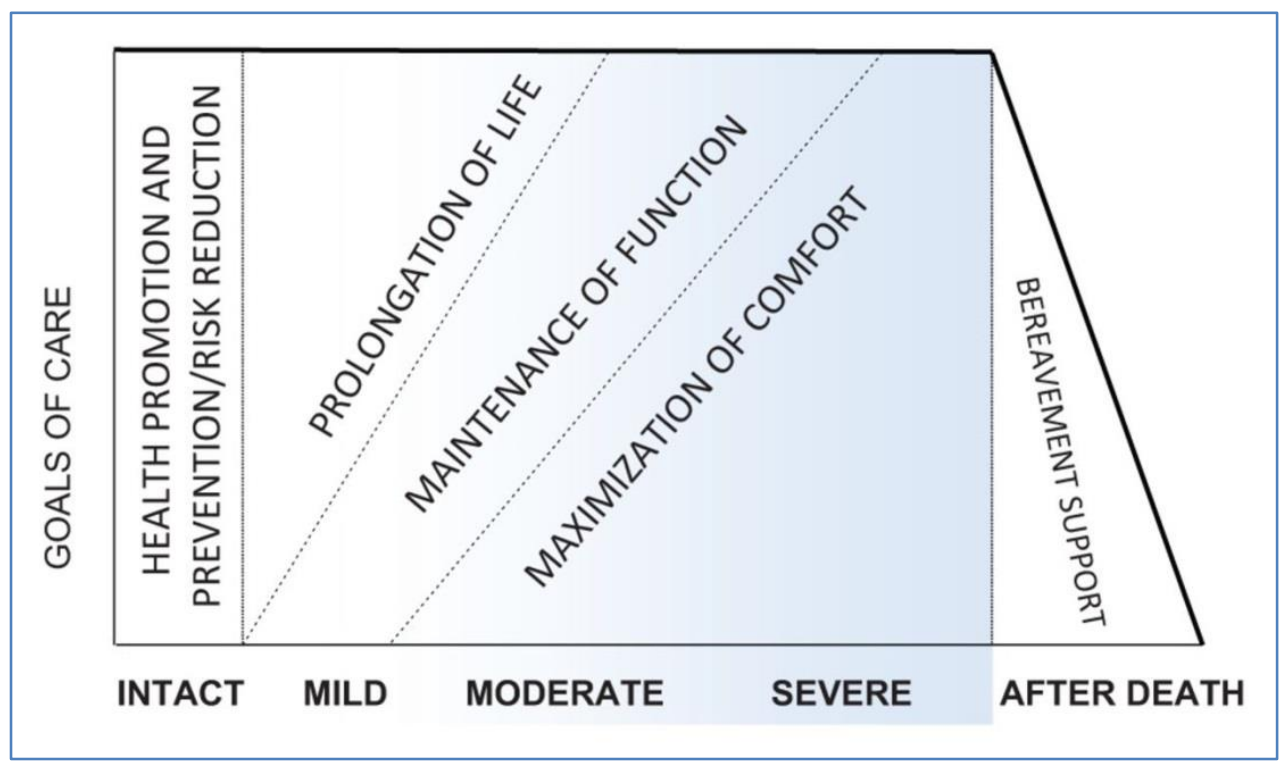

Figure 18. Model of palliative care in dementia; from European Association of Palliative Care White Paper

Figure reproduced from van der Steen et al (543) by creative commons license https://creativecommons.org/licenses/by-nc/3.0/

They acknowledge the vital role played by carers and family members who may experience distress, and "anticipatory" grief (544). Family carers are frequently "decision makers" and may make difficult and emotionally demanding choices at the end-of-life, for example, regarding feeding and resuscitation, as discussed in the caring and supporting sections above.

\section{Key challenges in end-of- life care for people with dementia and their families}

End-of-life care research has focussed on people with advanced dementia rather than people with less severe dementia dying from other conditions. Specifically, it is unknown how people in the earlier dementia stages with a terminal illness navigate services and make complex treatment decisions, and if they have equitable access to good end-of-life care.

Most symptoms people with advanced dementia experience can be managed by those with generalist knowledge of palliative care and good quality nursing. However, it is essential that staff have the skills and knowledge to consider the needs of people with dementia $(545 ; 546)$.

People with advanced dementia experience a range of symptoms, which may be poorly detected and under-treated (547). Pressure sores, agitation and swallowing difficulties are common and the 
total symptom burden is similar to those dying with cancer $(538 ; 548)$. People with advanced dementia are often immobile, bed bound, at risk of aspiration and have impaired immunological function increasing their risk of pneumonia, urinary and other infections (549). Assessment and management of pain is essential as, untreated, it leads to declining quality of life, depression and may worsen agitation and other neuropsychiatric symptoms (550). There are many tools to assess pain in dementia (551), however, they also measure distress and "discomfort" which may be caused by factors such as cold, poor positioning, boredom or lack of social contact (552) (553).

Using artificial nutrition and hydration (ANH) (including intravenous fluids and parenteral feeding) in advanced dementia is particularly difficult and emotive. There is little evidence that ANH reduces the risk of aspiration pneumonia, prolongs life or improves nutritional status or quality of life (554).

Difficulty swallowing and decreased appetite, sometimes secondary to lower calorie requirements, are common features of advanced dementia (555). Families are concerned that their relative will feel hungry or thirsty; the provision of food and assisting with eating is often a way to enact their care for their relative. Practices about using percutaneous endoscopic gastrostomy (PEG) and nasogastric tubes varies between countries (556;557), and across different US states (558), possibly because of legal differences

Directly transferring interventions and models from the cancer field may not work. In contrast to the cancer workforce, most end-of-life care for people with dementia is provided by care assistants in care homes; the most common setting in which people with dementia die(552). Good personcentred care requires a whole person approach and several multicomponent complex interventions and pathways have been developed. Training and educational programmes on end-of-life care for nursing home staff are effective in improving knowledge and increasing bereaved family members' satisfaction with end-of-life care $(559 ; 560)$. Research has focussed on specific interventions, such as pain management, or when not to treat e.g., with antibiotics, rather than active palliative interventions (561). Complex interventions taking into account variation between care homes and the need for coordinated multidisciplinary care have been developed but need further testing $(5 ; 559)$. Most people with dementia prefer to die in their usual place of residence and improving continuity of care could decrease costs by reducing emergency department visits and hospitalisations which usually do not prolong life but can be very distressing.

While advance care planning has been suggested as a way to improve choice, autonomy and ultimately end-of-life care, a person, even in dementia's earliest stages, may struggle to imagine their future self and make definitive plan, (562). There is little evidence whether advance care plans, made soon after the diagnosis of dementia change outcomes or improve the "quality of death". People with dementia, and their family and friends find advance care planning discussions helpful but value these as an ongoing process rather than committing an advance care plan to paper (563) (564). Assisted dying for people with dementia is controversial and emotive, raising complex legal and ethical issues. Legality varies by country. The main reason that carers of people with advanced dementia consider assisted dying is the person with dementia's distress (565). This provides a strong rationale for providing maximal comfort and quality of life as death approaches.

\section{Key points and recommendations}

People with dementia may be unable to communicate their needs, so assessment and management of pain and discomfort is key to providing good end-of-life care. There is prognostic uncertainty, so 
the priority is adopting a needs-based care approach focusing on the person with dementia and their carers. Optimal palliative care for people with dementia recognises the role of family members and that they may experience distress and "anticipatory" grief. Training and educating nursing home staff on end-of-life care is effective in improving knowledge and increasing satisfaction with such care in bereaved family members and should be routinely implemented.

\section{Delivering}

\section{Case management models for people with dementia}

Case management is delivered by a specific individual or a team to integrate the complex network of health and social care professionals needed in dementia and respond to patient needs, through an individualised, collaborative, evidence-based plan of care with and for patients and family needs (211). It usually includes standardised assessment, carer education, and implementation, of an individualised plan. Social workers, nurses or specialist dementia workers can be coordinators to achieve patient-centred care by and provide access to resources, plans of care, assessing environmental needs and educating and supporting carers, implementing plans, monitor and reassess $(566 ; 567)(568)$. There is variability on its content and implementation among and within countries (568). Case management is based on chronic disease management models, that improvement in care incorporates patient, provider, and system level interventions (569). It uses an inter-professional teams, including physicians, nurses, psychologists, physical and occupational therapists, and social worker to address patients' and families' complex medical, psychological and social needs (570)(571)(572). Additional support includes assisting with decisions about finances and healthcare and referral to key services such as transportation, home assistance, meal delivery and adult day programmes (572). Care management on the other hand refers to general co-ordination of care but the terms are often used synonymously (573).

Family carers often do not know about available services (574) so do not request or use them. The organisation of care provision differs between countries and services may be free at the point of delivery, or require individual purchase, sometimes with reimbursement. However, people with dementia use less healthcare even when freely available, than others with similar health needs; instead they use social care, and typically family carers provide more care rather than increase care access $(575 ; 576)$. Increasing rates of service utilisation by family carers would require professionals making the dementia care system 'visible' throughout dementia's course, so that the right support can be identified and accessed (577).

\section{Studies of case management models for people with dementia}

Table 3 shows case management approaches. Recent systematic reviews (568;578-580) and metaanalyses $(499 ; 581)$ of case management in dementia included 23 trials from nine countries. Over two thirds of the studies (70\%) were of poor or fair quality, and evaluated interventions that varied in content, duration (most 12-18 months), setting (e.g. primary care, social services), integration with health systems, care team composition, intensity and mode of contact, whether they interfaced with patients, carers, or both, and which outcomes targeted. Case management approaches also differ in the extent to which they are adapted to meet individual needs targeting specific outcomes (582) or use specific guidelines whereby the same intervention is offered to all individuals (583).

These reviews show that case management has a low to moderate effect on improving patient quality of life, and on adherence to practice recommendations and did not lead to decreased costs. 
They found case management reduced carer burden and depression (moderate effect size), but there was little evidence that these approaches benefit patients on outcomes such as neuropsychiatric symptoms, cognition, function or mortality (578-580).

Long-term care placement was the primary outcome in about half of the RCTs. Case management was associated with a low reduction on risk of nursing home admission up to 18 months (when intervention duration was $<2$ years) $(579 ; 580)$, but did not affect resource utilisation or healthcare costs over 1 year. However, continuity of care (patients seeing fewer different clinicians, despite their comorbidities) is associated with less hospital admissions and lower costs of care (584). Few studies have specifically examined cost-effectiveness $(578 ; 580)$..

Case management provided by social workers as part of collaborative care in the US reduced care inequalities (585). The US Care of Persons with dementia in their home Environment study (COPE), a multidisciplinary study with patients receiving healthcare and carers receiving advice found that at 4 months there was less functional dependence although this difference had disappeared at 9 months (586). Alternative models of case management for dementia, like the Maximizing Independence at Home model (MIND at Home), are emerging using well-trained, non-clinical staff as the front line coordinators, supported by nurses, physicians, and social worker, with preliminary evidence suggesting these models, which may be scalable with a larger potential workforce able to care for persons with dementia, have the potential to improve care for persons $(298 ; 582)$.

\section{Key points and recommendations}

Case management connects and facilitates access to different types of needed services for people with dementia. There is a lack of high-quality effectiveness and cost-effectiveness data. There is also heterogeneity between case management approaches, lack of manualised practice and standardisation, and limited information on how and what to implement. It should incorporate evidence-based interventions as best practice in dementia care. It may improve patient quality of life and reduce nursing home or hospital admissions for people with dementia. Making case management available, scalable and sustainable will require expanding and training the workforce.

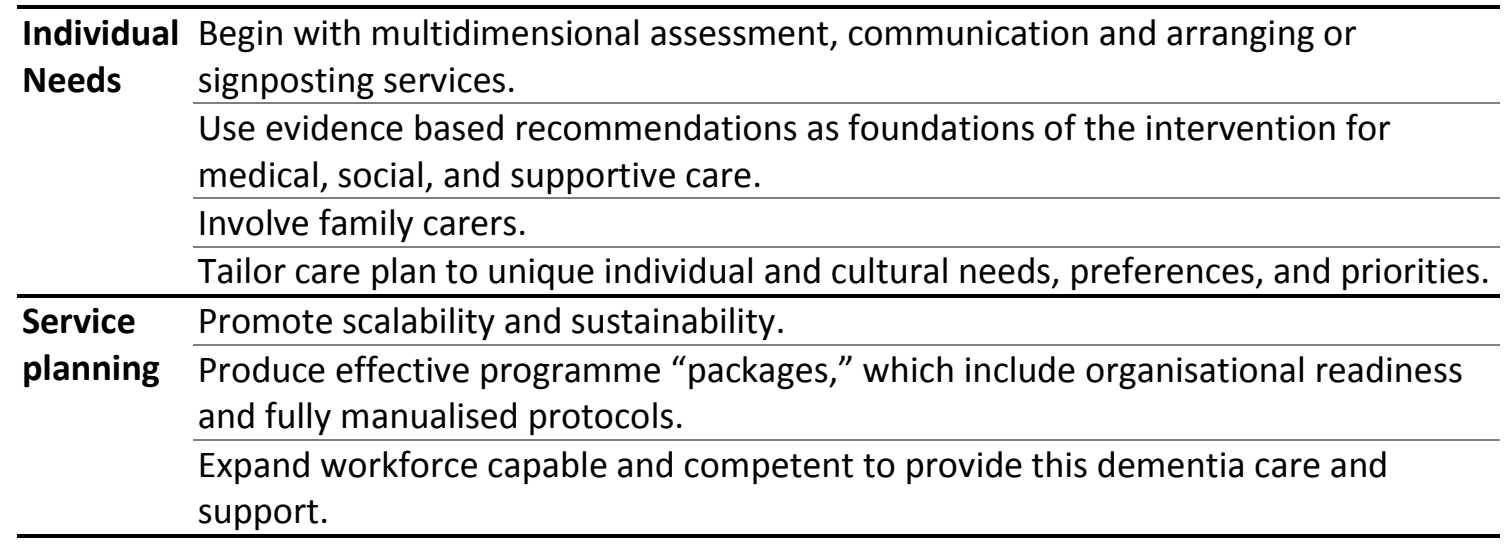

Table 3. Approaches to case management in dementia

\section{Care homes and assisted living}

Although most people with dementia are cared for by family members, many people with dementia eventually move into care homes when family carers are unable to manage their increasing care 
needs. Care homes may not offer specialist dementia services (587), despite around $80 \%$ of residents having dementia (588-591) (592;593) (594).

Care homes are highly complex and differ in terms of organisational characteristics (e.g. proprietary status, size of unit), processes (access to specialised dementia care ,case management or palliative care) and structures of care (hours of care provided per resident, level of expertise or diversity of workforce) (595). They differ in terms of practices, such as antipsychotic prescribing, indicating that provision of care is driven both by clinical need and the organisational culture of the care home (596;597).

People living in care homes usually have a lesser quality of life than those at home, possibly because they had more physical or neuropsychiatric symptoms or less support at home which led to their move (498) (598). Some residents' have more social support, reduced isolation and improved care when they move to a care home and their quality of life improves (599) (600). A systematic review of interventions found that it is likely that interventions that incorporate person-centred care, activity and sensory stimulation decrease agitation (595). However, a meta-analysis of care home interventions found that the evidence was not enough to recommend any particular programme or compare effectiveness (601).

Person-centred care can be taught to staff and increases job-satisfaction (602). The STAR (Staff Training in Assisted-living Residences) study was a pilot intervention with only limited evidence but initial positive results (302). It trained clinicians, family members, and other health care professionals to engage with the person through four manual-guided workshops, augmented by on-site sessions and leadership sessions. Residents hadg fewer affective symptoms and staff a less adverse reaction to residents' behavioural difficulties. It has now been translated into practice $(603 ; 604)$. Increasing international concern about high levels of psychotropic medication use, particularly antipsychotics (605), has led to decreased use for people with dementia as discussed above. Interventions such as educating and supporting care home staff or multicomponent interventions have reduced shortterm inappropriate prescribing of antipsychotic medications in care homes but evidence of longterm effectiveness and sustainability is still needed (606). However, a recent study in care homes which already had low levels of antipsychotic use, found that reducing antipsychotics, without adding other intervention, is not helpful for neuropsychiatric symptoms (607). Implementing effective interventions have required significant training and longer term supervision or working alongside care-home staff for a prolonged period(608).

Care transitions from acute care to care home settings require addressing communication barriers between hospitals and nursing homes and between families and care home staff to improve outcomes for patients by lowering incidence of both transfer and transfer-related harm, such as mistakes in medication $(559 ; 609 ; 610)(611)$.

\section{Leadership in care homes}

Leadership can play an important role in implementing evidence-based practice and is a key tool in facilitating care home changes (612). It can ensure consistent implementation and sustainability, instil values consistent with high quality care, such as cooperation between care home staff and healthcare professionals (613); ensure quality standards and procedures are in place (614); and foster a climate that recognises skills and advances employees' careers $(615 ; 616)$. This will require available resources and work is currently ongoing about how this can be achieved (424). 


\section{Assisted living}

Assisted living (extra-care sheltered housing, intermediate care housing, housing with care or assisted living residences) is an increasingly common option for people with dementia, who are unable to 'age in place', that is in their own home (617). Estimates indicate that $45 \%$ to $67 \%$ of residents of such facilities have dementia, of whom more than half have moderate-to-severe dementia and at least one neuropsychiatric symptom $(618 ; 619)$. People with dementia living in these settings often do not access treatment $(618 ; 620)$. Integration of dementia services in these settings, staff education and training, and monitoring of psychotropic medication may improve treatment and care for people with dementia (621).

There is increasing interest in 'home-like' residential care models and developing 'fit for the future' residential settings (622). Examples include the Eden Alternative and other small-scale facilities which are sometimes specifically designed for people with dementia (623). There is currently a lack of defined key characteristics of these models and information about outcomes (624). Some studies indicate that people with dementia might benefit from them in their physical functioning (625-627) however comparative-effectiveness and cost-effectiveness research is incomplete (624).

\section{Key points and recommendations}

Interventions in care homes require longer term working with professionals after the initial education in order to sustain the intervention and address and change organisational culture. A combination of communication strategies and clear procedures for increasing physical and social activity may reduce or prevent agitation in care homes.

\section{Technological innovations in dementia care}

Table 4 gives an overview of current and possible future uses of dementia-related devices.

The huge advances in the development of healthcare devices including electronic health records, portal technologies and wireless communications (628) which are likely to play a key role in dementia care in future. Given the progressive nature of dementia, certain devices may have a 'window' of usefulness to people with dementia and their carers (629). While somewhat overlapping, dementia healthcare technologies can be divided into five general categories:

1) Technologies for diagnosis and assessment; such as computerised neuropsychological assessments and telemedicine to facilitate examinations, testing and therapy in remote areas (630); 2) Monitoring; including sensors (motion; infrared; video; pressure, moisture; vital sign measurement and video) to detect changes in the person with dementia's environment or health status $(628 ; 630 ; 631)$;

3) Assistive- include cognitive aids (e.g. reminder systems for medication management); ADL assistance; and safety devices (e.g. electrical outlet shutoff devices) (628;630;631).

4) Therapeutic -include those that address communication, companionship and activity (628) (630). Despite recent interest in the animal assisted interventions in long-term care settings, often using social assistive robots (SARs) there are very few well-controlled studies $(632 ; 633)$

5) Carer supportive $(630 ; 634)$ - technology either to assist carers with the care of the person with dementia or support their own well-being $(630 ; 635 ; 636)$. 


\begin{tabular}{|c|c|c|}
\hline Purpose & $\begin{array}{l}\text { Type of health- } \\
\text { care device }\end{array}$ & Example of use \\
\hline \multirow{4}{*}{$\begin{array}{l}\text { Diagnosis } \\
\text { and } \\
\text { assessment }\end{array}$} & \multirow{2}{*}{$\begin{array}{l}\text { Computerised } \\
\text { diagnostic } \\
\text { assessment }\end{array}$} & Neuropsychological assessments \\
\hline & & Video-conferenced examinations \\
\hline & $\begin{array}{l}\text { Detecting } \\
\text { progression }\end{array}$ & Wearable sensors to detect changes in gait or ADLs \\
\hline & Virtual reality & Assessment of ADLs, e.g. meal preparation \\
\hline \multirow{6}{*}{ Monitoring } & \multirow{4}{*}{$\begin{array}{l}\text { Environmental } \\
\text { Sensors }\end{array}$} & Detection of changes in movement, e.g. falls \\
\hline & & Sensors to detect and intervene in environment, e.g. heat, gas \\
\hline & & GPS locating devices \\
\hline & & Remote viewing camera \\
\hline & \multirow{2}{*}{$\begin{array}{l}\text { Physiological } \\
\text { Sensors }\end{array}$} & $\begin{array}{l}\text { Devices measuring pulse, } \mathrm{BP}, \mathrm{O}_{2} \text { saturation, blood glucose, } \\
\text { sleep }\end{array}$ \\
\hline & & "Smart garments" with sensors which send biometric data \\
\hline \multirow{7}{*}{$\begin{array}{l}\text { Assistive } \\
\text { technology }\end{array}$} & \multirow{3}{*}{ Cognitive Aids } & Reminder systems, e.g. medication management \\
\hline & & $\begin{array}{l}\text { ADL prompting, e.g. tool that prompts user through } \\
\text { handwashing }\end{array}$ \\
\hline & & Cognitive training \\
\hline & ADL Assistance & Robots to assist eating, washing and mobility \\
\hline & \multirow{2}{*}{ Safety } & Electrical outlet shutoff devices \\
\hline & & Hands-free taps and water temperature sensors \\
\hline & Combination & $\begin{array}{l}\text { Robot to assist with care and monitor physiological or } \\
\text { environmental changes and send information to carers }\end{array}$ \\
\hline \multirow{4}{*}{$\begin{array}{l}\text { Therapeutic } \\
\text { technology }\end{array}$} & \multirow[t]{2}{*}{ Communication } & $\begin{array}{l}\text { Supporting reminiscence-based communication between } \\
\text { people with dementia and their carers }\end{array}$ \\
\hline & & Chat groups \\
\hline & Companionship & Robotic animals \\
\hline & Activity & $\begin{array}{l}\text { Technology to deliver music, messages, images and video } \\
\text { tailored to an individual's interests }\end{array}$ \\
\hline \multirow{4}{*}{$\begin{array}{l}\text { Carer } \\
\text { Supportive } \\
\text { Technology }\end{array}$} & Telemedicine & Video-conferencing with professionals \\
\hline & \multirow{2}{*}{$\begin{array}{l}\text { Online } \\
\text { information }\end{array}$} & Virtual assistance for managing challenges \\
\hline & & Web-based tools to support carer decision-making \\
\hline & Peer support & Carer online phone support groups \\
\hline
\end{tabular}

Table 4. Possible use for technological innovations in dementia care

\section{Challenges and priority areas for the future}

Technological innovations for people with dementia and their carers is an area of significant growth, but there are few rigorous RCT studies for most devices for people with dementia, with most research exploring feasibility, and acceptability (637), rather than clinical-effectiveness. The existing literature concentrates on technical aspects of delivery or physical disability (634). Many of these devices are not implemented and evaluated. Despite the potential applicability of technological innovations, there are important challenges to be addressed.

Ethical challenges: The aim of technological innovations should be to improve care without unacceptably increasing risks for people with dementia and their families. Preserving privacy and autonomy for the person with dementia is also important. While some devices have the potential to 
enhance safety, they also raise concerns in relation to replacing or reducing human contact (638). The development and use of devices used to restrict or restrain people with dementia raise additional concerns.

\section{Key points and recommendations}

Advances in the use and application of technological innovations may aid people with dementia to live in safe, stimulating and functionally enabling environments and support and assist carers and professionals in improving quality of care However, currently evidence on the effectiveness is lacking for most devices. Caution is therefore needed to protect people with dementia from overselling of ineffective and potentially unsafe devices. Technology is not a replacement for human contact.

\section{Conclusions}

Continued progress will build on what has long informed dementia care: to prevent the preventable, treat the treatable and care for both the person living with dementia and the carer. Here we have brought these strands together, informed by our understanding of the best evidence and explained the reasons for our conclusions. Evidence is always incomplete but we present the current evidence and the conclusions we have reached transparently. From this evidence and recognising that in each area, more must be done, we have suggested what can and should be done now.

Our recommendations are informed by the knowledge that dementia impairs cognition and therefore challenges the ability of people to make decisions for themselves, understand and communicate what they want and need. We therefore must take the utmost care and the necessary time to elicit the views of people with dementia and of their family carers.

Additionally, giving people information about what to do to prevent or treat dementia is an essential first step but is not enough. There is a responsibility, not just as professionals but as a society, to implement this evidence into interventions that are widely and effectively used for people with dementia and their families. Interventions have to be accessible, sustainable and, if possible, enjoyable or will be unused. Delivery of interventions will vary according to the health system, with some countries having healthcare free at point of delivery for all and others having to implement this as part of a programme. Interventions, which provide both the evidence and manuals with the necessary materials, are easier to implement and to alter according to the country in which they are used. It is important to consider who will deliver programmes and practicalities so that they are widely available to people with dementia and their families.

While dementia is due to brain pathology, people live with dementia in our societies, which should encounter, accept, contain and support them. This entails community design to foster safe affordable social activity and transportation, as well as societies in which people with dementia are accepted and integrated. Thus, while we recommend specific interventions to prevent dementia, diagnose it early, manage the cognitive and neuropsychiatric symptoms, support carers and improve living and dying with dementia, it is important that this health and social care takes place within, rather than separate from, society so we become truly dementia friendly. 


\section{Authors' contribution}

Gill Livingston (GL), Andrew Sommerlad (AS), Naaheed Mukadam (NM), Vasiliki Orgeta (VO) and Jonathan Huntley $(\mathrm{JH})$ drafted and redrafted the whole report.

NM, AS and GL conceived the new PAF calculation and NM led the statistical analysis.

Sergi Costafreda led the new meta-analysis for hearing impairment and dementia risk.

All authors contributed to sections of the reports and all revised the paper critically for important intellectual content.

$\mathrm{GL}$ is guarantor.

\section{Partners}

We are partnered by UCL, the Alzheimer's Society, UK; the Economic and Social Research Council and Alzheimer's Research UK and would like to thank them for financial help and attending our conferences. These organisations funded the fares, living accommodation and food for the commission meetings but had no role in the writing of the manuscript or the decision to submit it for publication.

\section{Acknowledgement}

We would like to thank Bernadette Courtney, Ephraim Robinson, Jacques Gianino, Nuj Monowari and Alexandra Ferrell from UCL for administrative help including managing finances, booking rooms and food and setting up a website. We would like to thank Sophie Naddell for proof reading.

\section{Declarations of interest}

DA reports personal fees from Eli Lilly, outside the submitted work.

CB reports grants and personal fees from Lundbeck, grants and personal fees from Acadia, personal fees from Roche, personal fees from Orion, personal fees from GSK, personal fees from Otusaka, personal fees from Heptares, personal fees from lilly, outside the submitted work.

SB reports grants and personal fees from Abbvie, personal fees and non-financial support from Lilly, personal fees from Eleusis, personal fees from Daval International, personal fees from BoehringerIngelheim, personal fees from Axovant, personal fees from Lundbeck, personal fees from Nutricia, outside the submitted work; he has been employed by the Department of Health for England.

EL reports other royalties from UpToDate, outside the submitted work.

GL reports grants from Alzheimer's Society, grants from ESRC, grants from ARUK, non-financial support from $U C L$, during the conduct of the study.

NF reports grants from NIHR, grants from Alzheimer's Research UK, grants from Medical Research Council (UK) during the conduct of the study; personal fees from Janssen/Pfizer, personal fees from IXICO, personal fees from Roche, other from Janssen Alzheimer's Immunotherapy Research and Development, personal fees from Lilly Research Laboratories (Avid), personal fees from Eli Lilly, personal fees from Novartis Pharma AG, personal fees from Sanofi, personal fees from GSK, outside the submitted work.

HK reports grants from National Institute of Nursing Research, outside the submitted work. 
K Rockwood has a patent null pending.

QS reports grants from National Institute on Aging, grants from Centers for Medicare and Medicaid, other from Broadmead Retirement Community, grants from BrightFocus Foundation, other from Welltower, grants from National Institute of Mental Health , grants from Hoffberger Foundation, outside the submitted work.

LS reports grants from NIA, grants from State of California, other from University of Southern California, during the conduct of the study; grants from Baxter, grants from Eli Lilly, grants from Forum, grants from Lundbeck, grants from Merck, grants from Novartis, grants from Roche/Genentech, grants from TauRx, personal fees from AC Immune, personal fees from Accera, personal fees from Avraham, personal fees from Boehringer Ingelheim, personal fees from Cerespir, personal fees from Cognition, personal fees from Forum, personal fees from Merck, personal fees from Neurim, personal fees from Roche, personal fees from Stemedica, personal fees from Takeda, personal fees from TauRx, personal fees from vTv, personal fees from Toyama/ FujiFilm, grants from Biogen, outside the submitted work.

$A B, C C, J C M, R H, J M, N M, V O, K$ Ritchie, ES, AS, and LT report no disclosures. 
(1) Alzheimer's Disease International. The Global Impact of Dementia 2013-2050: Policy Brief for Heads of Government. London: Alzheimer's Disease International; 2013.

(2) Prince, M, Wimo, A, Guerchet, M, Ali, GC, Wu, Y. T., and Prina M. World Alzheimer Report 2015 - The Global Impact of Dementia: An analysis of prevalence, incidence, cost and trends. London: Alzheimer's Disease International (ADI); 2015.

(3) Winblad B, Amouyel P, Andrieu S, Ballard C, Brayne C, Brodaty H et al. Defeating Alzheimer's disease and other dementias: a priority for European science and society. Lancet Neurol 2016 April;15(5):455-532.

(4) Prince M, Bryce R, Albanese E, Wimo A, Ribeiro W, Ferri CP. The global prevalence of dementia: a systematic review and metaanalysis. Alzheimers Dement 2013 January;9(1):63-75.

(5) Jones L, Candy B, Davis S, Elliott M, Gola A, Harrington J et al. Development of a model for integrated care at the end of life in advanced dementia: A whole systems UK-wide approach. Palliat Med 2016 March;30(3):279-95.

(6) Rocca WA, Petersen RC, Knopman DS, Hebert LE, Evans DA, Hall KS et al. Trends in the incidence and prevalence of Alzheimer's disease, dementia, and cognitive impairment in the United States. Alzheimers Dement 2011 January; 7(1):80-93.

(7) Matthews FE, Arthur A, Barnes LE, Bond J, Jagger C, Robinson L et al. A two-decade comparison of prevalence of dementia in individuals aged 65 years and older from three geographical areas of England: results of the Cognitive Function and Ageing Study I and II. Lancet 2013 October 26;382(9902):1405-12.

(8) Matthews FE, Stephan BC, Robinson L, Jagger C, Barnes LE, Arthur A et al. A two decade dementia incidence comparison from the Cognitive Function and Ageing Studies I and II. Nat Commun 2016;7:11398.

(9) World Health Organization. International Statistical Classification of Diseases and Related Health Problems 10th Revision. WHO; 2016.

(10) Stevens T, Livingston G, Kitchen G, Manela M, Walker Z, Katona C. Islington study of dementia subtypes in the community. Br J Psychiatry 2002 March;180:270-6.

(11) Mukadam N, Livingston G. Reducing the stigma associated with dementia: approaches and goals. Aging Health 2012;8(4):377-86.

(12) Blazer D. Neurocognitive disorders in DSM-5. Am J Psychiatry 2013 June;170(6):585-7.

(13) Sachdev PS, Blacker D, Blazer DG, Ganguli M, Jeste DV, Paulsen JS et al. Classifying neurocognitive disorders: the DSM-5 approach. Nat Rev Neurol 2014 November;10(11):634-42.

(14) Carone M, Asgharian M, Jewell NP. Estimating the lifetime risk of dementia in the Canadian elderly population using cross-sectional cohort survival data. J Am Stat Assoc 2014;109(505):24-35. 
(15) Song X, Mitnitski A, Rockwood K. Age-related deficit accumulation and the risk of late-life dementia. Alzheimers Res Ther 2014;6(5-8):54.

(16) Intzandt B, Black SE, Lanctot KL, Herrmann N, Oh P, Middleton LE. Is Cardiac Rehabilitation Exercise Feasible for People with Mild Cognitive Impairment? Can Geriatr J 2015 June;18(2):65-72.

(17) Cooper C, Ketley D, Livingston G. Systematic review and meta-analysis to estimate potential recruitment to dementia intervention studies. Int J Geriatr Psychiatry 2014 May;29(5):515-25.

(18) Brayne C, Gao L, Dewey M, Matthews FE. Dementia before death in ageing societies - The promise of prevention and the reality. Plos Medicine 2006 October;3(10):1922-30.

(19) Larson EB, Langa KM. The rising tide of dementia worldwide. Lancet 2008 August 9;372(9637):430-2.

(20) Tom SE, Hubbard RA, Crane PK, Haneuse SJ, Bowen J, McCormick WC et al. Characterization of dementia and Alzheimer's disease in an older population: updated incidence and life expectancy with and without dementia. Am J Public Health 2015 February;105(2):408-13.

(21) Manton KC, Gu XL, Ukraintseva SV. Declining prevalence of dementia in the U.S. elderly population. Adv Gerontol 2005;16:30-7.

(22) Langa KM, Larson EB, Karlawish JH, Cutler DM, Kabeto MU, Kim SY et al. Trends in the prevalence and mortality of cognitive impairment in the United States: is there evidence of a compression of cognitive morbidity? Alzheimers Dement 2008 March;4(2):134-44.

(23) Schrijvers EM, Verhaaren BF, Koudstaal PJ, Hofman A, Ikram MA, Breteler MM. Is dementia incidence declining?: Trends in dementia incidence since 1990 in the Rotterdam Study. Neurology 2012 May 8;78(19):1456-63.

(24) Qiu C, von SE, Backman L, Winblad B, Fratiglioni L. Twenty-year changes in dementia occurrence suggest decreasing incidence in central Stockholm, Sweden. Neurology 2013 May 14;80(20):1888-94.

(25) Satizabal CL, Beiser AS, Chouraki V, Chene G, Dufouil C, Seshadri S. Incidence of Dementia over Three Decades in the Framingham Heart Study. N Engl J Med 2016 February 11;374(6):523-32.

(26) Langa KM, Larson EB, Crimmins E. A Comparison of the Prevalence of Dementia in the United States in 2000 and 2012. JAMA Intern Med 2016 November 21.

(27) Chan KY, Wang W, Wu JJ, Liu L, Theodoratou E, Car J et al. Epidemiology of Alzheimer's disease and other forms of dementia in China, 1990-2010: a systematic review and analysis. Lancet 2013 June 8;381(9882):2016-23.

(28) Dodge HH, Buracchio TJ, Fisher GG, Kiyohara Y, Meguro K, Tanizaki Y et al. Trends in the prevalence of dementia in Japan. Int J Alzheimers Dis 2012;2012:956354.

(29) Okamura H, Ishii S, Ishii T, Eboshida A. Prevalence of dementia in Japan: a systematic review. Dement Geriatr Cogn Disord 2013;36(1-2):111-8. 
(30) Gao S, Ogunniyi A, Hall KS, Baiyewu O, Unverzagt FW, Lane KA et al. Dementia incidence declined in African-Americans but not in Yoruba. Alzheimers Dement 2016 March;12(3):244-51.

(31) Loef M, Walach H. Midlife obesity and dementia: meta-analysis and adjusted forecast of dementia prevalence in the United States and China. Obesity (Silver Spring) 2013 January;21(1):E51-E55.

(32) Norton S, Matthews FE, Barnes DE, Yaffe K, Brayne C. Potential for primary prevention of Alzheimer's disease: an analysis of population-based data. Lancet Neurol 2014 August;13(8):788-94.

(33) Skoog I, Vanmechelen E, Andreasson LA, Palmertz B, Davidsson P, Hesse C et al. A population-based study of tau protein and ubiquitin in cerebrospinal fluid in 85-year-olds: relation to severity of dementia and cerebral atrophy, but not to the apolipoprotein E4 allele. Neurodegeneration 1995 December;4(4):433-42.

(34) Lim A, Tsuang D, Kukull W, Nochlin D, Leverenz J, McCormick W et al. Cliniconeuropathological correlation of Alzheimer's disease in a community-based case series. J Am Geriatr Soc 1999 May;47(5):564-9.

(35) Larson EB. Illnesses causing dementia in the very elderly. N Engl J Med 1993 January 21;328(3):203-5.

(36) Snowdon DA, Greiner LH, Mortimer JA, Riley KP, Greiner PA, Markesbery WR. Brain infarction and the clinical expression of Alzheimer disease: The Nun Study. Journal of the American Medical Association 1997;277(10):12-817.

(37) Langa KM, Foster NL, Larson EB. Mixed dementia: emerging concepts and therapeutic implications. JAMA 2004 December 15;292(23):2901-8.

(38) Sonnen JA, Postupna N, Larson EB, Crane PK, Rose SE, Montine KS et al. Pathologic correlates of dementia in individuals with Lewy body disease. Brain Pathol 2010 May;20(3):654-9.

(39) Cholerton B, Larson EB, Baker LD, Craft S, Crane PK, Millard SP et al. Neuropathologic correlates of cognition in a population-based sample. J Alzheimers Dis 2013;36(4):699-709.

(40) Sonnen JA, Santa CK, Hemmy LS, Woltjer R, Leverenz JB, Montine KS et al. Ecology of the aging human brain. Arch Neurol 2011 August;68(8):1049-56.

(41) SantaCruz KS, Sonnen JA, Pezhouh MK, Desrosiers MF, Nelson PT, Tyas SL. Alzheimer disease pathology in subjects without dementia in 2 studies of aging: the Nun Study and the Adult Changes in Thought Study. J Neuropathol Exp Neurol 2011 October;70(10):83240.

(42) White L, Petrovitch H, Hardman J, Nelson J, Davis DG, Ross GW et al. Cerebrovascular pathology and dementia in autopsied Honolulu-Asia Aging Study participants. Ann N Y Acad Sci 2002 November;977:9-23.

(43) Larson EB, Yaffe K, Langa KM. New insights into the dementia epidemic. N Engl J Med 2013 December 12;369(24):2275-7. 
(44) Joint Programme for Neurodegenerative Research. Longitudinal cohort studies in neurodegeneration research: Repot of the JPND action group. 2013 Sep 1.

(45) Niu H, Alvarez-Alvarez I, Guillen-Grima F, Aguinaga-Ontoso I. Prevalence and incidence of Alzheimer's disease in Europe: A meta-analysis. Neurologia 2016 April 26.

(46) Stern Y. Cognitive reserve in ageing and Alzheimer's disease. Lancet Neurology 2012 November;11(11):1006-12.

(47) Stern Y. Cognitive Reserve: Implications for Assessment and Intervention. Folia Phoniatrica et Logopaedica 2013;65(2):49-54.

(48) Amieva H, Mokri H, Le Goff M, Meillon C, Jacqmin-Gadda H, Foubert-Samier A et al. Compensatory mechanisms in higher-educated subjects with Alzheimer's disease: a study of 20 years of cognitive decline. Brain 2014 April;137:1167-75.

(49) Strydom A, Livingston G, King M, Hassiotis A. Prevalence of dementia in intellectual disability using different diagnostic criteria. British Journal of Psychiatry 2007;191:150-7.

(50) Laditka JN, Laditka SB, Cornman CB, Porter CN, Davis DR, Mintzer J. Notably higher rates of vascular risk factors and dementia among African Americans in South Carolina: opportunities for public health intervention. J S C Med Assoc 2008 October;104(7):219-22.

(51) Adelman S, Blanchard M, Livingston G. A systematic review of the prevalence and covariates of dementia or relative cognitive impairment in the older African-Caribbean population in Britain. Int J Geriatr Psychiatry 2009 July;24(7):657-65.

(52) Adelman S, Blanchard M, Rait G, Leavey G, Livingston G. Prevalence of dementia in African-Caribbean compared with UK-born White older people: two-stage cross-sectional study. Br J Psychiatry 2011 August;199(2):119-25.

(53) Borenstein A, Mortimer J. Alzheimer's Disease: Life Course Perspectives on Risk Reduction. 1st Edition ed. Academic Press; 2016.

(54) Larson EB. Prospects for delaying the rising tide of worldwide, late-life dementias. Int Psychogeriatr 2010 December;22(8):1196-202.

(55) Moceri VM, Kukull WA, Emanual I, van BG, Starr JR, Schellenberg GD et al. Using census data and birth certificates to reconstruct the early-life socioeconomic environment and the relation to the development of Alzheimer's disease. Epidemiology 2001 July;12(4):3839.

(56) Valenzuela MJ, Sachdev P. Brain reserve and dementia: a systematic review. Psychol Med 2006 April;36(4):441-54.

(57) National Institute for Clinical Excellence. Dementia, disability and frailty in later life - midlife approaches to delay or prevent onset. 2015 Oct 20.

(58) Daviglus ML, Bell CC, Berrettini W, Bowen PE, Connolly ES, Jr., Cox NJ et al. NIH state-ofthe-science conference statement: Preventing Alzheimer's disease and cognitive decline. NIH Consens State Sci Statements 2010 April 28;27(4):1-30. 
(59) Barnes DE, Yaffe K. The projected effect of risk factor reduction on Alzheimer's disease prevalence. Lancet Neurol 2011 September;10(9):819-28.

(60) Kuiper JS, Zuidersma M, Oude Voshaar RC, Zuidema SU, van den Heuvel ER, Stolk RP et al. Social relationships and risk of dementia: A systematic review and meta-analysis of longitudinal cohort studies. Ageing Res Rev 2015 July;22:39-57.

(61) ESRC GROWING OLDER PROGRAM. Loneliness, social isolation and living alone in later life. 2003.

(62) Hayden JA, van der Windt DA, Cartwright JL, Cote P, Bombardier C. Assessing bias in studies of prognostic factors. Ann Intern Med 2013 February 19;158(4):280-6.

(63) Deal JA, Betz J, Yaffe K, Harris T, Purchase-Helzner E, Satterfield S et al. Hearing Impairment and Incident Dementia and Cognitive Decline in Older Adults: The Health ABC Study. 2016.

(64) Lin FR, Metter EJ, O'Brien RJ, Resnick SM, Zonderman AB, Ferrucci L. Hearing loss and incident dementia. 2011;68(2):214-20.

(65) Gallacher J, llubaera V, Ben-Shlomo Y, Bayer A, Fish M, Babisch W et al. Auditory threshold, phonologic demand, and incident dementia. 2012;79(15):1583-90.

(66) Rockhill B, Newman B, Weinberg C. Use and misuse of population attributable fractions. American Journal of Public Health 1998 January;88(1):15-9.

(67) Health and Social Care Information Centre. Health Survey for England 2014 : health, social care and lifestyles : summary of key findings. 2015.

(68) Kaiser HF. The application of electronic computers to factor analysis. Educational and Psychological Measurement 1960;20:141-51.

(69) Ritchie K, Carriere I, Ritchie CW, Berr C, Artero S, Ancelin ML. Designing prevention programmes to reduce incidence of dementia: prospective cohort study of modifiable risk factors. BMJ 2010;341:c3885.

(70) Casserly I, Topol E. Convergence of atherosclerosis and Alzheimer's disease: inflammation, cholesterol, and misfolded proteins. Lancet 2004 April 3;363(9415):1139-46.

(71) Qiu C, Sigurdsson S, Zhang Q, Jonsdottir MK, Kjartansson O, Eiriksdottir G et al. Diabetes, markers of brain pathology and cognitive function: the Age, Gene/Environment Susceptibility-Reykjavik Study. Ann Neurol 2014 January;75(1):138-46.

(72) Hoyer S, Blum-Degen D, Bernstein HG, Engelsberger S, Humrich J, Laufer S et al. Brain insulin and insulin receptors in aging and sporadic Alzheimer's disease. Journal of Neural Transmission 1998;105(4-5):423-38.

(73) Biessels GJ, Staekenborg S, Brunner E, Brayne C, Scheltens P. Risk of dementia in diabetes mellitus: a systematic review. Lancet Neurology 2006 January;5(1):64-74.

(74) Andel R, Crowe M, Pedersen NL, Fratiglioni L, Johansson B, Gatz M. Physical exercise at midlife and risk of dementia three decades later: A population-based study of Swedish 
twins. Journals of Gerontology Series A-Biological Sciences and Medical Sciences 2008 January;63(1):62-6.

(75) Vaughan S, Wallis M, Polit D, Steele M, Shum D, Morris N. The effects of multimodal exercise on cognitive and physical functioning and brain-derived neurotrophic factor in older women: a randomised controlled trial. Age and Ageing 2014 September;43(5):623-9.

(76) Leckie RL, Oberlin LE, Voss MW, Prakash RS, Szabo-Reed A, Chaddock-Heyman L et al. BDNF mediates improvements in executive function following a 1-year exercise intervention. Front Hum Neurosci 2014;8:985.

(77) Young J, Angevaren M, Rusted J, Tabet N. Aerobic exercise to improve cognitive function in older people without known cognitive impairment. Cochrane Database Syst Rev 2015;4:CD005381.

(78) Valenzuela MJ. Brain reserve and the prevention of dementia. Current Opinion in Psychiatry 2008;21(3):May-302.

(79) Lin FR, Metter EJ, O'Brien RJ, Resnick SM, Zonderman AB, Ferrucci L. Hearing loss and incident dementia. Arch Neurol 2011 February;68(2):214-20.

(80) Lin FR, Ferrucci L, Metter EJ, An Y, Zonderman AB, Resnick SM. Hearing loss and cognition in the Baltimore Longitudinal Study of Aging. Neuropsychology 2011 November;25(6):76370 .

(81) Lin FR. Hearing loss and cognition among older adults in the United States. J Gerontol A Biol Sci Med Sci 2011 October;66(10):1131-6.

(82) Deal JA, Sharrett AR, Albert MS, Coresh J, Mosley TH, Knopman D et al. Hearing impairment and cognitive decline: a pilot study conducted within the atherosclerosis risk in communities neurocognitive study. 2015;181(9):680-90.

(83) Kiely KM, Gopinath B, Mitchell P, Luszcz M, Anstey KJ. Cognitive, health, and sociodemographic predictors of longitudinal decline in hearing acuity among older adults. 2012;67(9):997-1003.

(84) Fritze T, Teipel S, +ôv+íri A, Kilimann I, Witt G, Doblhammer G. Hearing Impairment Affects Dementia Incidence. An Analysis Based on Longitudinal Health Claims Data in Germany. 2016;11(7):e0156876.

(85) Gurgel RK, Ward PD, Schwartz S, Norton MC, Foster NL, Tschanz JT. Relationship of hearing loss and dementia: a prospective, population-based study. 2014;35(5):775-81.

(86) Amieva H+, Ouvrard C, Giulioli C, Meillon C+, Rullier L, Dartigues JF. Self-Reported Hearing Loss, Hearing Aids, and Cognitive Decline in Elderly Adults: A 25-Year Study. 2015;63(10):2099-104.

(87) Valentijn SAM, Van Boxtel MPJ, Van Hooren SAH, Bosma H, Beckers HJM, Ponds RW et al. Change in sensory functioning predicts change in cognitive functioning: Results from a 6year follow-up in the Maastricht Aging Study. 2005;53(3):374-80. 
(88) Hong T, Mitchell P, Burlutsky G, Liew G, Wang JJ. Visual Impairment, Hearing Loss and Cognitive Function in an Older Population: Longitudinal Findings from the Blue Mountains Eye Study. 2016;11(1):e0147646.

(89) Lin MY, Gutierrez PR, Stone KL, Yaffe K, Ensrud KE, Fink HA et al. Vision impairment and combined vision and hearing impairment predict cognitive and functional decline in older women. 2004;52(12):1996-2002.

(90) Scholes S, Mindel J. Health Survey for England 2014: Health, social care and lifestyles. In: Craig R, Fuller E, Mindell J, editors. Chapter 4: Hearing. 2014.

(91) McCoy SL, Tun PA, Cox LC, Colangelo M, Stewart RA, Wingfield A. Hearing loss and perceptual effort: Downstream effects on older adults memory for speech. The Quarterly Journal of Experimental Psychology Section A 2005;58(1):22-33.

(92) Huang CQ, Dong BR, Lu ZC, Yue JR, Liu QX. Chronic diseases and risk for depression in old age: a meta-analysis of published literature. 2010;9(2):131-41.

(93) Gopinath B, Wang JJ, Schneider J, Burlutsky G, Snowdon J, McMahon CM et al. Depressive symptoms in older adults with hearing impairments: the Blue Mountains Study. 2009;57(7):1306-8.

(94) Lin FR, Albert M. Hearing loss and dementia - who is listening? Aging \& mental health 2014;18(6):2014-673.

(95) Bernabei R, Bonuccelli U, Maggi S, Marengoni A, Martini A, Memo M et al. Hearing loss and cognitive decline in older adults: questions and answers. 2014;26(6):567-73.

(96) Davis A, Smith P, Ferguson M, Stephens D, Gianopoulos I. Acceptability, benefit and costs of early screening for hearing disability: a study of potential screening tests and models. 2007;11(42):1-294.

(97) Hartley D, Rochtchina E, Newall P, Golding M, Mitchell P. Use of hearing aids and assistive listening devices in an older Australian population. 2010;21(10):642-53.

(98) Gates GA. Central presbycusis: an emerging view. 2012;147(1):1-2.

(99) Gates GA, Beiser A, Rees TS, D'Agostino RB, Wolf PA. Central auditory dysfunction may precede the onset of clinical dementia in people with probable Alzheimer's disease. 2002;50(3):482-8.

(100) Sinha UK, Hollen KM, Rodriguez R, Miller CA. Auditory system degeneration in Alzheimer's disease. Neurology 1993 April;43(4):779-85.

(101) Gates GA, Cobb JL, Linn RT, Rees T, Wolf PA, D'Agostino RB. Central auditory dysfunction, cognitive dysfunction, and dementia in older people. Arch Otolaryngol Head Neck Surg 1996 February;122(2):161-7.

(102) Nieman CL, Marrone N, Mamo SK, Betz J, Choi JS, Contrera KJ et al. The Baltimore HEARS Pilot Study: An Affordable, Accessible, Community-Delivered Hearing Care Intervention. The Gerontologist. In press 2016. 
(103) Sofi F, Valecchi D, Bacci D, Abbate R, Gensini GF, Casini A et al. Physical activity and risk of cognitive decline: a meta-analysis of prospective studies. J Intern Med 2011 January;269(1):107-17.

(104) Hamer M, Chida Y. Physical activity and risk of neurodegenerative disease: a systematic review of prospective evidence. Psychol Med 2009 January;39(1):3-11.

(105) de LC, Guimaraes-Pinheiro C, Maseda A, Lorenzo T, Millan-Calenti JC. Effects of physical exercise interventions in frail older adults: a systematic review of randomized controlled trials. BMC Geriatr 2015 December 2;15:154.

(106) Blake H, Mo P, Malik S, Thomas S. How effective are physical activity interventions for alleviating depressive symptoms in older people? A systematic review. Clin Rehabil 2009 October;23(10):873-87.

(107) Almeida OP, Khan KM, Hankey GJ, Yeap BB, Golledge J, Flicker L. 150 minutes of vigorous physical activity per week predicts survival and successful ageing: a population-based 11year longitudinal study of 12201 older Australian men. Br J Sports Med 2014 February;48(3):220-5.

(108) Wilson PW, D'Agostino RB, Sullivan L, Parise H, Kannel WB. Overweight and obesity as determinants of cardiovascular risk: the Framingham experience. Archives of Internal Medicine 2002 September 9;162(16):1867-72.

(109) Luchsinger JA, Gustafson DR. Adiposity and Alzheimer's disease. Current Opinion in Clinical Nutrition and Metabolic Care 2009;12(1):January-21.

(110) Yaffe K. Metabolic syndrome and cognitive disorders: Is the sum greater than its parts? Alzheimer Disease and Associated Disorders 2007;21(2):April/June-171.

(111) Swan GE, Lessov-Schlaggar CN. The effects of tobacco smoke and nicotine on cognition and the brain. Neuropsychology Review 2007;17(3):September-273.

(112) World Health Organization. WHO global report on trends in tobacco smoking 2000-2025. geneva: World Health Organization; 2015.

(113) Dotson VM, Beydoun MA, Zonderman AB. Recurrent depressive symptoms and the incidence of dementia and mild cognitive impairment. Neurology 2010;75(1):06-34.

(114) Alexopoulos GS. Vascular disease, depression, and dementia. Journal of the American Geriatrics Society 2003;51(8):01-1180.

(115) Sheline YI, West T, Yarasheski K, Swarm R, Jasielec MS, Fisher JR et al. An antidepressant decreases CSF Abeta production in healthy individuals and in transgenic AD mice. Sci Transl Med 2014 May 14;6(236):236re4.

(116) Morkem R, Barber D, Williamson T, Patten SB. A Canadian Primary Care Sentinel Surveillance Network Study Evaluating Antidepressant Prescribing in Canada From 2006 to 2012. Can J Psychiatry 2015 December;60(12):564-70.

(117) Olfson M, Marcus SC. National patterns in antidepressant medication treatment. Arch Gen Psychiatry 2009 August;66(8):848-56. 
(118) Yang YC, Boen C, Gerken K, Li T, Schorpp K, Harris KM. Social relationships and physiological determinants of longevity across the human life span. Proceedings of the National Academy of Sciences of the United States of America 2016 January 19;113(3):578-83.

(119) Hemingway H, Marmot M. Evidence based cardiology: psychosocial factors in the aetiology and prognosis of coronary heart disease. Systematic review of prospective cohort studies. BMJ 1999 May 29;318(7196):1460-7.

(120) Santini ZI, Koyanagi A, Tyrovolas S, Mason C, Haro JM. The association between social relationships and depression: a systematic review. Journal of Affective Disorders 2015 April 1;175:53-65.

(121) Scarmeas N, Stern Y, Mayeux R, Manly JJ, Schupf N, Luchsinger JA. Mediterranean diet and mild cognitive impairment. Archives of Neurology 2009;66(2):February-225.

(122) Khaw KT, Wareham N, Bingham S, Welch A, Luben R, Day N. Combined impact of health behaviours and mortality in men and women: the EPIC-Norfolk prospective population study. Plos Medicine 2008 January 8;5(1):e12.

(123) Crane PK, Gibbons LE, Dams-O'Connor K, Trittschuh E, Leverenz JB, Keene CD et al. Association of Traumatic Brain Injury With Late-Life Neurodegenerative Conditions and Neuropathologic Findings. JAMA Neurol 2016 July 11.

(124) Perry DC, Sturm VE, Peterson MJ, Pieper CF, Bullock T, Boeve BF et al. Association of traumatic brain injury with subsequent neurological and psychiatric disease: a metaanalysis. J Neurosurg 2016 February;124(2):511-26.

(125) Fleminger S, Oliver DL, Lovestone S, Rabe-Hesketh S, Giora A. Head injury as a risk factor for Alzheimer's disease: the evidence 10 years on; a partial replication. Journal of Neurology, Neurosurgery \& Psychiatry 2003;74(7):857-62.

(126) Guo Z, Cupples LA, Kurz A, Auerbach SH, Volicer L, Chui H et al. Head injury and the risk of AD in the MIRAGE study. Neurology 2000;54(6):1316-23.

(127) Mac Donald CL, Johnson AM, Cooper D, Nelson EC, Werner NJ, Shimony JS et al. Detection of blast-related traumatic brain injury in U.S. military personnel. N Engl J Med 2011 June 2;364(22):2091-100.

(128) McKee AC, Stern RA, Nowinski CJ, Stein TD, Alvarez VE, Daneshvar DH et al. The spectrum of disease in chronic traumatic encephalopathy. Brain 2013 January;136(Pt 1):43-64.

(129) Institute of Medicine. Long-term Consequences of Traumatic Brain Injury. Washington, DC: National Academies Press; 2009.

(130) Bialystok E, Craik Fl, Klein R, Viswanathan M. Bilingualism, aging, and cognitive control: evidence from the Simon task. Psychology and aging 2004;19(2):290.

(131) Sanders AE, Hall CB, Katz MJ, Lipton RB. Non-native language use and risk of incident dementia in the elderly. Journal of Alzheimer's Disease 2012;29(1):99-108. 
(132) Zahodne LB, Schofield PW, Farrell MT, Stern Y, Manly JJ. Bilingualism does not alter cognitive decline or dementia risk among Spanish-speaking immigrants. Neuropsychology 2014;28(2):238.

(133) Chen H, Kwong JC, Copes R, Tu K, Villeneuve PJ, van DA et al. Living near major roads and the incidence of dementia, Parkinson's disease, and multiple sclerosis: a population-based cohort study. Lancet 2017 January 4.

(134) Hill A B. The environment and disease: association or causation? Proc R Soc Med 1965;58:295-300.

(135) Lin FR, Metter EJ, O'Brien RJ, Resnick SM, Zonderman AB, Ferrucci L. Hearing loss and incident dementia. Archives of Neurology 2011;68(2):February-220.

(136) Kakigi A, Hirakawa H, Harel N, Mount RJ, Harrison RV. Tonotopic mapping in auditory cortex of the adult chinchilla with amikacin-induced cochlear lesions. Audiology 2000 May;39(3):153-60.

(137) Schwaber MK, Garraghty PE, Kaas JH. Neuroplasticity of the adult primate auditory cortex following cochlear hearing loss. Am J Otol 1993 May;14(3):252-8.

(138) Cheung SW, Bonham BH, Schreiner CE, Godey B, Copenhaver DA. Realignment of interaural cortical maps in asymmetric hearing loss. J Neurosci 2009 May 27;29(21):706578.

(139) Lazarov O, Robinson J, Tang YP, Hairston IS, Korade-Mirnics Z, Lee VM et al. Environmental enrichment reduces Abeta levels and amyloid deposition in transgenic mice. Cell 2005 March 11;120(5):701-13.

(140) Dunbar R. Coevolution of neocortical size, group size and language in humans. Behav Brain Sci 1993;16(4):681-94.

(141) Jorm AF, Korten AE, Henderson AS. The Prevalence of Dementia - A Quantitative Integration of the Literature. Acta Psychiatrica Scandinavica 1987 November;76(5):465-79.

(142) Imtiaz B, Tolppanen AM, Kivipelto M, Soininen H. Future directions in Alzheimer's disease from risk factors to prevention. Biochem Pharmacol 2014 April 15;88(4):661-70.

(143) Andrieu S, Coley N, Lovestone S, Aisen PS, Vellas B. Prevention of sporadic Alzheimer's disease: lessons learned from clinical trials and future directions. Lancet Neurol 2015 September;14(9):926-44.

(144) Williams JW, Plassman BL, Burke J, Benjamin S. Preventing Alzheimer's disease and cognitive decline. Evid Rep Technol Assess (Full Rep ) 2010 April;(193):1-727.

(145) Peters R, Beckett N, Forette F, Tuomilehto J, Clarke R, Ritchie C et al. Incident dementia and blood pressure lowering in the Hypertension in the Very Elderly Trial cognitive function assessment (HYVET-COG): a double-blind, placebo controlled trial. Lancet Neurol 2008 August; $7(8): 683-9$.

(146) McGuinness B, Todd S, Passmore P, Bullock R. Blood pressure lowering in patients without prior cerebrovascular disease for prevention of cognitive impairment and dementia. Cochrane Database Syst Rev 2009;(4):CD004034. 
(147) Forette F, Seux ML, Staessen JA, Thijs L, Birkenhager WH, Babarskiene MR et al. Prevention of dementia in randomised double-blind placebo-controlled Systolic Hypertension in Europe (Syst-Eur) trial. Lancet 1998 October 24;352(9137):1347-51.

(148) Moll van Charante EP, Richard E, Eurelings L, van Dalen J, Ligthart SA, van Bussel EF et al. Effectiveness of a 6-year multidomain vascular care intervention to prevent dementia (preDIVA): a cluster-randomised controlled trial. The Lancet 2016.

(149) Krause T, Lovibond K, Caulfield M, McCormack T, Williams B, Guideline Development Group. Management of hypertension: summary of NICE guidance. BMJ 2011;343:d4891.

(150) Martin BK, Szekely C, Brandt J, Piantadosi S, Breitner JC, Craft S et al. Cognitive function over time in the Alzheimer's Disease Anti-inflammatory Prevention Trial (ADAPT): results of a randomized, controlled trial of naproxen and celecoxib. Arch Neurol 2008 July;65(7):896-905.

(151) Gold M, Alderton C, Zvartau-Hind M, Egginton S, Saunders AM, Irizarry M et al. Rosiglitazone monotherapy in mild-to-moderate Alzheimer's disease: results from a randomized, double-blind, placebo-controlled phase III study. Dement Geriatr Cogn Disord 2010;30(2):131-46.

(152) McGuiness B, Craig D, Bullock R, Passmore P. Statins for the prevention of dementia. Cochrane Database of Systematic Reviews 2016;CD003160(1).

(153) Ancelin ML, Ritchie K. Lifelong endocrine fluctuations and related cognitive disorders. Curr Pharm Des 2005;11(32):4229-52.

(154) McCarrey AC, Resnick SM. Postmenopausal hormone therapy and cognition. Horm Behav 2015 August;74:167-72.

(155) Valls-Pedret C, Sala-Vila A, Serra-Mir M, Corella D, de la Torre R, Martinez-Gonzalez MA et al. Mediterranean Diet and Age-Related Cognitive Decline: A Randomized Clinical Trial. JAMA Intern Med 2015 July;175(7):1094-103.

(156) Ball K, Berch DB, Helmers KF, Jobe JB, Leveck MD, Marsiske $M$ et al. Effects of cognitive training interventions with older adults: a randomized controlled trial. JAMA 2002 November 13;288(18):2271-81.

(157) Rebok GW, Ball K, Guey LT, Jones RN, Kim HY, King JW et al. Ten-year effects of the advanced cognitive training for independent and vital elderly cognitive training trial on cognition and everyday functioning in older adults. J Am Geriatr Soc 2014 January;62(1):16-24.

(158) Corbett A, Owen A, Hampshire A, Grahn J, Stenton R, Dajani S et al. The Effect of an Online Cognitive Training Package in Healthy Older Adults: An Online Randomized Controlled Trial. J Am Med Dir Assoc 2015 November 1;16(11):990-7.

(159) Ngandu T, Lehtisalo J, Solomon A, Levalahti E, Ahtiluoto S, Antikainen R et al. A 2 year multidomain intervention of diet, exercise, cognitive training, and vascular risk monitoring versus control to prevent cognitive decline in at-risk elderly people (FINGER): a randomised controlled trial. Lancet 2015 June 6;385(9984):2255-63. 
(160) Kelly ME, Loughrey D, Lawlor BA, Robertson IH, Walsh C, Brennan S. The impact of exercise on the cognitive functioning of healthy older adults: a systematic review and metaanalysis. Ageing Res Rev 2014 July;16:12-31.

(161) Smith PJ, Blumenthal JA, Hoffman BM, Cooper H, Strauman TA, Welsh-Bohmer K et al. Aerobic exercise and neurocognitive performance: a meta-analytic review of randomized controlled trials. Psychosom Med 2010 April;72(3):239-52.

(162) Fiatarone Singh MA, Gates N, Saigal N, Wilson GC, Meiklejohn J, Brodaty H et al. The Study of Mental and Resistance Training (SMART) study-resistance training and/or cognitive training in mild cognitive impairment: a randomized, double-blind, double-sham controlled trial. J Am Med Dir Assoc 2014 December;15(12):873-80.

(163) Brown BM, Peiffer JJ, Martins RN. Multiple effects of physical activity on molecular and cognitive signs of brain aging: can exercise slow neurodegeneration and delay Alzheimer's disease? Mol Psychiatry 2013 August;18(8):864-74.

(164) Jensen CS, Hasselbalch SG, Waldemar G, Simonsen AH. Biochemical Markers of Physical Exercise on Mild Cognitive Impairment and Dementia: Systematic Review and Perspectives. Front Neurol 2015;6:187.

(165) Duzel E, van PH, Sendtner M. Can physical exercise in old age improve memory and hippocampal function? Brain 2016 March;139(Pt 3):662-73.

(166) Erickson KI, Voss MW, Prakash RS, Basak C, Szabo A, Chaddock L et al. Exercise training increases size of hippocampus and improves memory. Proc Natl Acad Sci U S A 2011 February 15;108(7):3017-22.

(167) Global Council on Brain Health. The Brain-Body Connection: GCBH Recommendations on Physical Activity and Brain Health. 2016.

(168) Sundstrom A, Westerlund O, Kotyrlo E. Marital status and risk of dementia: a nationwide population-based prospective study from Sweden. BMJ Open 2016;6(1):e008565.

(169) Cohen-Mansfield J, Shmotkin D, Goldberg S. Loneliness in old age: longitudinal changes and their determinants in an Israeli sample. Int Psychogeriatr 2009 December;21(6):116070 .

(170) Carlson MC, Saczynski JS, Rebok GW, Seeman T, Glass TA, McGill S et al. Exploring the effects of an "everyday" activity program on executive function and memory in older adults: Experience Corps. Gerontologist 2008 December;48(6):793-801.

(171) Cohen-Mansfield J, Cohen R, Buettner L, Eyal N, Jakobovits H, Rebok G et al. Interventions for older persons reporting memory difficulties: a randomized controlled pilot study. Int J Geriatr Psychiatry 2015 May;30(5):478-86.

(172) Kivipelto M, Solomon A, Ahtiluoto S, Ngandu T, Lehtisalo J, Antikainen R et al. The Finnish Geriatric Intervention Study to Prevent Cognitive Impairment and Disability (FINGER): study design and progress. Alzheimers Dement 2013 November;9(6):657-65.

(173) Kivipelto M, Ngandu T, Laatikainen T, Winblad B, Soininen H, Tuomilehto J. Risk score for the prediction of dementia risk in 20 years among middle aged people: a longitudinal, population-based study. Lancet Neurol 2006 September;5(9):735-41. 
(174) Richard E, Van den Heuvel E, Moll van Charante EP, Achthoven L, Vermeulen M, Bindels PJ et al. Prevention of dementia by intensive vascular care (PreDIVA): a cluster-randomized trial in progress. Alzheimer Dis Assoc Disord 2009 July;23(3):198-204.

(175) Schneider LS. Reduce vascular risk to prevent dementia. The Lancet 2016.

(176) Vellas B, Carrie I, Gillette-Guyonnet S, Touchon J, Dantoine T, Dartigues JF et al. MAPT study: a multidomain approach for preventing Alzheimer's disease: design and baseline data. J Prev Alzheimers Dis 2014 June;1(1):13-22.

(177) Healthy Aging Through Internet Counselling in the Elderly (HATICE). 16-5-2016. Ref Type: Internet Communication

(178) Jack CR, Jr., Knopman DS, Jagust WJ, Shaw LM, Aisen PS, Weiner MW et al. Hypothetical model of dynamic biomarkers of the Alzheimer's pathological cascade. Lancet Neurol 2010 January;9(1):119-28.

(179) Ritchie K, Carriere I, Berr C, Amieva H, Dartigues JF, Ancelin ML et al. The clinical picture of alzheimer's disease in the decade before diagnosis: clinical and biomarker trajectories. J Clin Psychiatry 2016 March;77(3):e305-e311.

(180) Ritchie K, Ritchie C, Yaffe K, Skoog I, Scarmeas N. Is late-onset Alzheimer's disease really a disease of mid-life. Alzheimer's \& Dementia: Translational Research \& Clinical Interventions 2015 September 1;1(2):122-30.

(181) Dubois B, Feldman HH, Jacova C, Hampel H, Molinuevo JL, Blennow K et al. Advancing research diagnostic criteria for Alzheimer's disease: the IWG-2 criteria. Lancet Neurol 2014 June;13(6):614-29.

(182) Jack CR, Jr., Albert MS, Knopman DS, McKhann GM, Sperling RA, Carrillo MC et al. Introduction to the recommendations from the National Institute on Aging-Alzheimer's Association workgroups on diagnostic guidelines for Alzheimer's disease. Alzheimers Dement 2011 May;7(3):257-62.

(183) Dubois B, Hampel H, Feldman HH, Scheltens P, Aisen P, Andrieu S et al. Preclinical Alzheimer's disease: Definition, natural history, and diagnostic criteria. Alzheimers Dement 2016 March;12(3):292-323.

(184) Ames D, Burns A, O'Brien J. Dementia. 5th ed. Oxford: OUP; 2016.

(185) Chetelat G, La JR, Villain N, Perrotin A, de LS, V, Eustache F et al. Amyloid imaging in cognitively normal individuals, at-risk populations and preclinical Alzheimer's disease. Neuroimage Clin 2013;2:356-65.

(186) Villemagne VL, Burnham S, Bourgeat P, Brown B, Ellis KA, Salvado O et al. Amyloid beta deposition, neurodegeneration, and cognitive decline in sporadic Alzheimer's disease: a prospective cohort study. Lancet Neurol 2013 April;12(4):357-67.

(187) Rowe CC, Villemagne VL. Amyloid imaging with PET in early Alzheimer disease diagnosis. Med Clin North Am 2013 May;97(3):377-98. 
(188) Dickerson BC, Wolk DA. Biomarker-based prediction of progression in $\mathrm{MCl}$ : Comparison of $A D$ signature and hippocampal volume with spinal fluid amyloid-beta and tau. Front Aging Neurosci 2013;5:55.

(189) Villemagne VL, Pike KE, Chetelat G, Ellis KA, Mulligan RS, Bourgeat P et al. Longitudinal assessment of Abeta and cognition in aging and Alzheimer disease. Ann Neurol 2011 January;69(1):181-92.

(190) Villemagne VL, Rowe CC. Long night's journey into the day: amyloid-beta imaging in Alzheimer's disease. J Alzheimers Dis 2013;33 Suppl 1:S349-S359.

(191) Alzforum: Networking for a cure. 12-5-2016.

Ref Type: Internet Communication

(192) Ritchie CW, Molinuevo JL, Truyen L, Satlin A, Van der Geyten S, Lovestone S. Development of interventions for the secondary prevention of Alzheimer's dementia: the European Prevention of Alzheimer's Dementia (EPAD) project. Lancet Psychiatry 2016 February;3(2):179-86.

(193) Ritchie CW, Ritchie K. The PREVENT study: a prospective cohort study to identify mid-life biomarkers of late-onset Alzheimer's disease. BMJ Open 2012;2(6).

(194) Weiner MW, Aisen PS, Jack CR, Jr., Jagust WJ, Trojanowski JQ, Shaw L et al. The Alzheimer's disease neuroimaging initiative: progress report and future plans. Alzheimers Dement 2010 May;6(3):202-11.

(195) Bateman RJ, Xiong C, Benzinger TL, Fagan AM, Goate A, Fox NC et al. Clinical and biomarker changes in dominantly inherited Alzheimer's disease. N Engl J Med 2012 August 30;367(9):795-804.

(196) Reiman EM, Langbaum JB, Tariot PN, Lopera F, Bateman RJ, Morris JC et al. CAP-advancing the evaluation of preclinical Alzheimer disease treatments. Nat Rev Neurol 2016 January;12(1):56-61.

(197) DeCarli C. Mild cognitive impairment: prevalence, prognosis, aetiology, and treatment. Lancet Neurol 2003 January;2(1):15-21.

(198) Graham JE, Rockwood K, Beattie BL, Eastwood R, Gauthier S, Tuokko H et al. Prevalence and severity of cognitive impairment with and without dementia in an elderly population. Lancet 1997 June 21;349(9068):1793-6.

(199) Petersen RC. Mild cognitive impairment as a diagnostic entity. J Intern Med 2004 September;256(3):183-94.

(200) Lopez OL, Kuller LH, Becker JT, Dulberg C, Sweet RA, Gach HM et al. Incidence of dementia in mild cognitive impairment in the cardiovascular health study cognition study. Arch Neurol 2007 March;64(3):416-20.

(201) Ganguli M, Dodge HH, Shen C, DeKosky ST. Mild cognitive impairment, amnestic type: an epidemiologic study. Neurology 2004 July 13;63(1):115-21. 
(202) Mitchell AJ, Shiri-Feshki M. Rate of progression of mild cognitive impairment to dementia - meta-analysis of 41 robust inception cohort studies. Acta Psychiatrica Scandinavica 2009;119(4):252-65.

(203) Tschanz JT, Welsh-Bohmer KA, Lyketsos CG, Corcoran C, Green RC, Hayden K et al. Conversion to dementia from mild cognitive disorder - The Cache County Study. Neurology 2006 July 25;67(2):229-34.

(204) Petersen RC, Stevens JC, Ganguli M, Tangalos EG, Cummings JL, DeKosky ST. Practice parameter: early detection of dementia: mild cognitive impairment (an evidence-based review). Report of the Quality Standards Subcommittee of the American Academy of Neurology. Neurology 2001 May 8;56(9):1133-42.

(205) Albert MS, DeKosky ST, Dickson D, Dubois B, Feldman HH, Fox NC et al. The diagnosis of mild cognitive impairment due to Alzheimer's disease: Recommendations from the National Institute on Aging-Alzheimer's Association workgroups on diagnostic guidelines for Alzheimer's disease. Alzheimers \& Dementia 2011 May;7(3):270-9.

(206) Cooper C, Sommerlad A, Lyketsos CG, Livingston G. Modifiable predictors of dementia in mild cognitive impairment: A systematic review and meta-analysis. American Journal of Psychiatry 2015;172(4):01-334.

(207) Ritchie K, Ancelin ML, Beaino E, Portet F, Brickman AM, Dartigues JF et al. Retrospective identification and characterization of mild cognitive impairment from a prospective population cohort. Am J Geriatr Psychiatry 2010 August;18(8):692-700.

(208) Artero S, Ancelin ML, Portet F, Dupuy A, Berr C, Dartigues JF et al. Risk profiles for mild cognitive impairment and progression to dementia are gender specific. J Neurol Neurosurg Psychiatry 2008 September;79(9):979-84.

(209) Ismail Z, Smith EE, Geda Y, Sultzer D, Brodaty H, Smith G et al. Neuropsychiatric symptoms as early manifestations of emergent dementia: provisional diagnostic criteria for mild behavioral impairment. Alzheimer's \& dementia 2016;12(2):195-202.

(210) Apostolova LG, Cummings JL. Neuropsychiatric manifestations in mild cognitive impairment: A systematic review of the literature. Dementia and Geriatric Cognitive Disorders 2008;25(2):115-26.

(211) National Institute for Clinical Excellence. Dementia: supporting people with dementia and their carers in health and social care. Clinical guideline CG42. 2006 Nov 22.

(212) Reijnders J, van HC, van BM. Cognitive interventions in healthy older adults and people with mild cognitive impairment: a systematic review. Ageing Res Rev 2013 January;12(1):263-75.

(213) Cooper C, Li R, Lyketsos C, Livingston G. Treatment for mild cognitive impairment: systematic review. Br J Psychiatry 2013 September;203(3):255-64.

(214) Gates N, Fiatarone Singh MA, Sachdev PS, Valenzuela M. The effect of exercise training on cognitive function in older adults with mild cognitive impairment: a meta-analysis of randomized controlled trials. Am J Geriatr Psychiatry 2013 November;21(11):1086-97. 
(215) van Uffelen JG, Chinapaw MJ, van MW, Hopman-Rock M. Walking or vitamin B for cognition in older adults with mild cognitive impairment? A randomised controlled trial. $\mathrm{Br}$ J Sports Med 2008 May;42(5):344-51.

(216) Sevigny J, Chiao P, Bussiere T, Weinreb PH, Williams L, Maier M et al. The antibody aducanumab reduces Abeta plaques in Alzheimer's disease. Nature 2016;537(7618):50-6.

(217) Prins ND, van der Flier WA, Knol DL, Fox NC, Brashear HR, Nye JS et al. de. Alzheimer's research \& therapy 2014;6(4):1.

(218) De Beaumont L, Pelleieux S, Lamarre-Th $+{ }^{\circledR}$ roux L, Dea D, Poirier J. Butyrylcholinesterase K

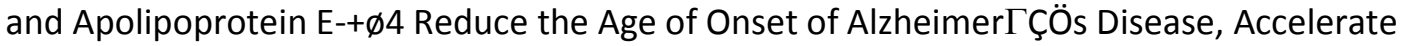
Cognitive Decline, and Modulate Donepezil Response in Mild Cognitively Impaired Subjects. Journal of Alzheimer's Disease 2016;(Preprint):1-10.

(219) Imbimbo BP, Solfrizzi V, Panza F. Are NSAIDs useful to treat Alzheimer's disease or mild cognitive impairment? Front Aging Neurosci 2010;2(19).

(220) Steenland K, Zhao L, Goldstein FC, Levey Al. Statins and cognitive decline in older adults with normal cognition or mild cognitive impairment. J Am Geriatr Soc 2013 September;61(9):1449-55.

(221) Petersen RC, Thomas RG, Grundman M, Bennett D, Doody R, Ferris S et al. Vitamin E and donepezil for the treatment of mild cognitive impairment. N Engl J Med 2005 June 9;352(23):2379-88.

(222) de Jager CA, Oulhaj A, Jacoby R, Refsum H, Smith AD. Cognitive and clinical outcomes of homocysteine-lowering B-vitamin treatment in mild cognitive impairment: a randomized controlled trial. Int J Geriatr Psychiatry 2012 June;27(6):592-600.

(223) DeKosky ST, Williamson JD, Fitzpatrick AL, Kronmal RA, Ives DG, Saxton JA et al. Ginkgo biloba for prevention of dementia: a randomized controlled trial. JAMA 2008 November 19;300(19):2253-62.

(224) Snitz BE, O'Meara ES, Carlson MC, Arnold AM, Ives DG, Rapp SR et al. Ginkgo biloba for preventing cognitive decline in older adults: a randomized trial. JAMA 2009 December 23;302(24):2663-70.

(225) Vellas B, Coley NF, Ousset PJ FAU - Berrut G, Berrut GF, Dartigues JF FAU - Dubois B, Dubois BF et al. Long-term use of standardised Ginkgo biloba extract for the prevention of Alzheimer's disease (GuidAge): a randomised placebo-controlled trial. Lancet Neurol 12 A.D.;11(10):851-9.

(226) Department of Health. Dementia: A state of the nation report on dementia care and support in England. 2013 Nov 1.

(227) Burns A, Robert P. The National Dementia strategy in England. BMJ 2009;338:b931.

(228) Mukadam N, Livingston G, Rantell K, Rickman S. Diagnostic rates and treatment of dementia before and after launch of a national dementia policy: an observational study using English national databases. BMJ open 2014;4(1). 
(229) Gitlin LN, Winter L, Burke J, Chernett N, Dennis MP, Hauck WW. Tailored activities to manage neuropsychiatric behaviors in persons with dementia and reduce caregiver burden: a randomized pilot study. Am J Geriatr Psychiatry 2008 March;16(3):229-39.

(230) Wilson JM, Jungner YG. [Principles and practice of mass screening for disease]. Bol Oficina Sanit Panam 1968 October;65(4):281-393.

(231) Shenkin SD, Russ TC, Ryan TM, MacLullich AM. Screening for dementia and other causes of cognitive impairment in general hospital in-patients. Age Ageing 2014 March;43(2):166-8.

(232) Who Cares Wins. Improving the outcome for older people admitted to the general hospital: guidelines for the development of liaison mental health services for older people. London: Royal College of Psychiatrists; 2005.

(233) Department of Health. Living well with dementia: A National Dementia Strategy . 2009.

(234) Prince, M., Bryce, R., and Ferri, C. P. World Alzheimer Report 2011: The benefits of early diagnosis and intervention. London: Alzheimer's Disease International, 11 A.D.

(235) Mormont E, Jamart J, Jacques D. Symptoms of depression and anxiety after the disclosure of the diagnosis of Alzheimer disease. J Geriatr Psychiatry Neurol 2014

December;27(4):231-6.

(236) Bunn F, Goodman C, Sworn K, Rait G, Brayne C, Robinson L et al. Psychosocial factors that shape patient and carer experiences of dementia diagnosis and treatment: a systematic review of qualitative studies. PLoS Med 2012 October;9(10):e1001331.

(237) Livingston G, Leavey G, Manela M, Livingston D, Rait G, Sampson E et al. Making decisions for people with dementia who lack capacity: qualitative study of family carers in UK. British Medical Journal 2010 August 18;341.

(238) Olafsdottir M, Foldevi M, Marcusson J. Dementia in primary care: why the low detection rate? Scand J Prim Health Care 2001 September;19(3):194-8.

(239) Livingston G, Johnston K, Katona C, Paton J, Lyketsos CG. Systematic review of psychological approaches to the management of neuropsychiatric symptoms of dementia. American Journal of Psychiatry 2005 November;162(11):1996-2021.

(240) Gitlin LN, Belle SH, Burgio LD, Czaja SJ, Mahoney D, Gallagher-Thompson D et al. Effect of multicomponent interventions on caregiver burden and depression: the REACH multisite initiative at 6-month follow-up. Psychol Aging 2003 September;18(3):361-74.

(241) Mahoney R, Regan C, Katona C, Livingston G. Anxiety and depression in family caregivers of people with Alzheimer disease: the LASER-AD study. Am J Geriatr Psychiatry 2005 September;13(9):795-801.

(242) Livingston G, Barber J, Rapaport P, Knapp M, Griffin M, King D et al. Clinical effectiveness of a manual based coping strategy programme (START, STrAtegies for RelaTives) in promoting the mental health of carers of family members with dementia: pragmatic randomised controlled trial. BMJ 2013;347:f6276.

(243) Pinner G, Bouman WP. Attitudes of patients with mild dementia and their carers towards disclosure of the diagnosis. Int Psychogeriatr 2003 September;15(3):279-88. 
(244) Mukadam N, Livingston G. Reducing the stigma associated with dementia: approaches and goals. Aging Health 2012;8(4):377-86.

(245) Hansen EC, Hughes C, Routley G, Robinson AL. General practitioners' experiences and understandings of diagnosing dementia: factors impacting on early diagnosis. Soc Sci Med 2008 December;67(11):1776-83.

(246) Rait G, Walters K, Bottomley C, Petersen I, lliffe S, Nazareth I. Survival of people with clinical diagnosis of dementia in primary care: cohort study. British Medical Journal 2010 August 5;341.

(247) Mukadam N, Cooper C, Livingston G. A systematic review of ethnicity and pathways to care in dementia. Int J Geriatr Psychiatry 2011;26(1):12-20.

(248) Berwald S, Roche M, Adelman S, Mukadam N, Livingston G. Black African and Caribbean British Communities' Perceptions of Memory Problems: "We Don't Do Dementia.". PLoS One 2016;11(4):e0151878.

(249) Mukadam N, Cooper C, Kherani N, Livingston G. A systematic review of interventions to detect dementia or cognitive impairment. Int J Geriatr Psychiatry 2015 January;30(1):3245.

(250) Mate KE, Magin PJ, Brodaty H, Stocks NP, Gunn J, Disler PB et al. An evaluation of the additional benefit of population screening for dementia beyond a passive case-finding approach. Int J Geriatr Psychiatry 2016 March 14.

(251) Livingston G, Baio G, Sommerlad A, Delusignan S, Poulimenos S, Morris S et al. Effectiveness of an Intervention to facilitate Timely Diagnosis of Dementia: Cluster Randomised Controlled Trial. PLoS Med. In press 2016.

(252) Laver K, Cumming RG, Dyer SM, Agar MR, Anstey KJ, Beattie E et al. Clinical practice guidelines for dementia in Australia. Med J Aust 2016 March 21;204(5):191-3.

(253) Kambugu A, Thompson J, Hakim J, Tumukunde D, van Oosterhout JJ, Mwebaze R et al. Neurocognitive Function at the First-Line Failure and on the Second-Line Antiretroviral Therapy in Africa: Analyses From the EARNEST Trial. J Acquir Immune Defic Syndr 2016 April 15;71(5):506-13.

(254) Velayudhan L, Ryu SH, Raczek M, Philpot M, Lindesay J, Critchfield M et al. Review of brief cognitive tests for patients with suspected dementia. International psychogeriatrics / IPA 2014;26(8):1247-62.

(255) Folstein MF, Folstein SE, McHugh PR. "Mini-mental state". A practical method for grading the cognitive state of patients for the clinician. J Psychiatr Res 1975 November;12(3):18998.

(256) Martin R, O'Neill D. Taxing your memory. Lancet 2009 June 13;373(9680):2009-10.

(257) Hsieh S, Schubert S, Hoon C, Mioshi E, Hodges JR. Validation of the Addenbrooke's Cognitive Examination III in frontotemporal dementia and Alzheimer's disease. Dement Geriatr Cogn Disord 2013;36(3-4):242-50. 
(258) Nasreddine ZS, Phillips N, Chertkow H. Normative data for the Montreal Cognitive Assessment (MoCA) in a population-based sample. Neurology 2012 March 6;78(10):765-6.

(259) Nasreddine ZS, Phillips NA, Bedirian V, Charbonneau S, Whitehead V, Collin I et al. The Montreal Cognitive Assessment, MoCA: a brief screening tool for mild cognitive impairment. J Am Geriatr Soc 2005 April;53(4):695-9.

(260) Storey JE, Rowland JT, Basic D, Conforti DA, Dickson HG. The Rowland Universal Dementia Assessment Scale (RUDAS): a multicultural cognitive assessment scale. Int Psychogeriatr 2004 March;16(1):13-31.

(261) Gauthier S, Patterson C, Chertkow H, Gordon M, Herrmann N, Rockwood K et al. Recommendations of the 4th Canadian Consensus Conference on the Diagnosis and Treatment of Dementia (CCCDTD4). Canadian geriatrics journal 2012;15(4):120-6.

(262) Harper L, Barkhof F, Scheltens P, Schott JM, Fox NC. An algorithmic approach to structural imaging in dementia. J Neurol Neurosurg Psychiatry 2014 June;85(6):692-8.

(263) Schott JM, Warren JD, Barkhof F, Rossor MN, Fox NC. Suspected early dementia. BMJ 2011;343:d5568.

(264) Scheltens P, Fox N, Barkhof F, De CC. Structural magnetic resonance imaging in the practical assessment of dementia: beyond exclusion. Lancet Neurol 2002 May;1(1):13-21.

(265) Fox NC, Schott JM. Imaging cerebral atrophy: normal ageing to Alzheimer's disease. Lancet 2004 January 31;363(9406):392-4.

(266) O'Brien JT, Paling S, Barber R, Williams ED, Ballard C, McKeith IG et al. Progressive brain atrophy on serial MRI in dementia with Lewy bodies, $A D$, and vascular dementia. Neurology 2001 May 22;56(10):1386-8.

(267) Duara R, Loewenstein DA, Potter E, Appel J, Greig MT, Urs R et al. Medial temporal lobe atrophy on MRI scans and the diagnosis of Alzheimer disease. Neurology 2008 December 9;71(24):1986-92.

(268) Burton EJ, Barber R, Mukaetova-Ladinska EB, Robson J, Perry RH, Jaros E et al. Medial temporal lobe atrophy on MRI differentiates Alzheimer's disease from dementia with Lewy bodies and vascular cognitive impairment: a prospective study with pathological verification of diagnosis. Brain 2009 January;132(Pt 1):195-203.

(269) O'Brien JT, Thomas A. Vascular dementia. Lancet 2015 October 24;386(10004):1698-706.

(270) Arvanitakis Z, Capuano AW, Leurgans SE, Bennett DA, Schneider JA. Relation of cerebral vessel disease to Alzheimer's disease dementia and cognitive function in elderly people: a cross-sectional study. Lancet Neurol 2016 June 13;15(9):934-43.

(271) Foster NL, Heidebrink JL, Clark CM, Jagust WJ, Arnold SE, Barbas NR et al. FDG-PET improves accuracy in distinguishing frontotemporal dementia and Alzheimer's disease. Brain 2007 October;130(Pt 10):2616-35.

(272) Medicare National Coverage Determinations Manual. 2016. 
(273) McCleery J, Morgan S, Bradley KM, Noel-Storr AH, Ansorge O, Hyde C. Dopamine transporter imaging for the diagnosis of dementia with Lewy bodies. Cochrane Database Syst Rev 2015;1:CD010633.

(274) Vijverberg EG, Wattjes MP, Dols A, Krudop WA, Moller C, Peters A et al. Diagnostic Accuracy of MRI and Additional [18F]FDG-PET for Behavioral Variant Frontotemporal Dementia in Patients with Late Onset Behavioral Changes. J Alzheimers Dis 2016 June 30;53(4):1287-97.

(275) McKeith I, O'Brien J, Walker Z, Tatsch K, Booij J, Darcourt J et al. Sensitivity and specificity of dopamine transporter imaging with 123I-FP-CIT SPECT in dementia with Lewy bodies: a phase III, multicentre study. Lancet Neurol 2007 April;6(4):305-13.

(276) Hyare H, So PW, Brandner S, Collinge J, Parkes HG. MRI detection of prion protein plaques in variant Creutzfeldt-Jakob disease. Neurology 2015 April 7;84(14):1498-9.

(277) McCleery J, Morgan S, Bradley KM, Noel-Storr AH, Ansorge O, Hyde C. Dopamine transporter imaging for the diagnosis of dementia with Lewy bodies. Cochrane Database Syst Rev 2015;1:CD010633.

(278) O'Brien JT, Firbank MJ, Davison C, Barnett N, Bamford C, Donaldson C et al. 18F-FDG PET and perfusion SPECT in the diagnosis of Alzheimer and Lewy body dementias. J Nucl Med 2014 December;55(12):1959-65.

(279) Johnson KA, Minoshima S, Bohnen NI, Donohoe KJ, Foster NL, Herscovitch P et al. Appropriate use criteria for amyloid PET: a report of the Amyloid Imaging Task Force, the Society of Nuclear Medicine and Molecular Imaging, and the Alzheimer's Association. Alzheimers Dement 2013 January;9(1):e-16.

(280) Villemagne VL. Amyloid imaging: Past, present and future perspectives. Ageing Res Rev 2016 January 28;95-106.

(281) Knopman DS, DeKosky ST, Cummings JL, Chui H, Corey-Bloom J, Relkin N et al. Practice parameter: diagnosis of dementia (an evidence-based review). Report of the Quality Standards Subcommittee of the American Academy of Neurology. Neurology 2001 May 8;56(9):1143-53.

(282) Hort J, O'Brien JT, Gainotti G, Pirttila T, Popescu BO, Rektorova I et al. EFNS guidelines for the diagnosis and management of Alzheimer's disease. Eur J Neurol 2010 October;17(10):1236-48.

(283) Fagan AM, Xiong C, Jasielec MS, Bateman RJ, Goate AM, Benzinger TL et al. Longitudinal change in CSF biomarkers in autosomal-dominant Alzheimer's disease. Sci Transl Med 2014 March 5;6(226):226ra30.

(284) Falahati F, Fereshtehnejad SM, Religa D, Wahlund LO, Westman E, Eriksdotter M. The use of $\mathrm{MRI}, \mathrm{CT}$ and lumbar puncture in dementia diagnostics: data from the SveDem Registry. Dement Geriatr Cogn Disord 2015;39(1-2):81-91.

(285) Menendez-Gonzalez M. Routine lumbar puncture for the early diagnosis of Alzheimer's disease. Is it safe? Front Aging Neurosci 2014;6:65. 
(286) Llorens F, Schmitz M, Ferrer I, Zerr I. CSF biomarkers in neurodegenerative and vascular dementias. Prog Neurobiol 2016 March;138-140:36-53.

(287) Olsson B, Lautner R, Andreasson U, Ohrfelt A, Portelius E, Bjerke $\mathrm{M}$ et al. CSF and blood biomarkers for the diagnosis of Alzheimer's disease: a systematic review and metaanalysis. Lancet Neurol 2016 April 8.

(288) Oeckl P, Steinacker P, Feneberg E, Otto M. Neurochemical biomarkers in the diagnosis of frontotemporal lobar degeneration: An update. J Neurochem 2016 May 17.

(289) Snowden JS, Adams J, Harris J, Thompson JC, Rollinson S, Richardson A et al. Distinct clinical and pathological phenotypes in frontotemporal dementia associated with MAPT, PGRN and C9orf72 mutations. Amyotroph Lateral Scler Frontotemporal Degener 2015;16(7-8):497-505.

(290) Blennow K, Johansson A, Zetterberg H. Diagnostic value of 14-3-3beta immunoblot and Ttau/P-tau ratio in clinically suspected Creutzfeldt-Jakob disease. Int J Mol Med 2005 December;16(6):1147-9.

(291) Valcarcel-Nazco C, Perestelo-Perez L, Molinuevo JL, Mar J, Castilla I, Serrano-Aguilar P. Cost-effectiveness of the use of biomarkers in cerebrospinal fluid for Alzheimer's disease. $J$ Alzheimers Dis 2014;42(3):777-88.

(292) McKhann GM, Knopman DS, Chertkow H, Hyman BT, Jack CR, Jr., Kawas CH et al. The diagnosis of dementia due to Alzheimer's disease: recommendations from the National Institute on Aging-Alzheimer's Association workgroups on diagnostic guidelines for Alzheimer's disease. Alzheimers Dement 2011 May;7(3):263-9.

(293) American Academy of Neurology. Detection, diagnosis and management of dementia: AAN Guideline summary for clinicians. 2004 Jan 1.

(294) Brouwers N, Sleegers K, Van BC. Molecular genetics of Alzheimer's disease: an update. Ann Med 2008;40(8):562-83.

(295) Corder EH, Saunders AM, Strittmatter WJ, Schmechel DE, Gaskell PC, Small GW et al. Gene dose of apolipoprotein $\mathrm{E}$ type 4 allele and the risk of Alzheimer's disease in late onset families. Science 1993 August 13;261(5123):921-3.

(296) Chen Q, Schubert D. Presenilin-interacting proteins. Expert Rev Mol Med 2002 August;4(19):1-18.

(297) Sorbi S, Hort J, Erkinjuntti T, Fladby T, Gainotti G, Gurvit H et al. EFNS-ENS Guidelines on the diagnosis and management of disorders associated with dementia. Eur J Neurol 2012 September;19(9):1159-79.

(298) Tanner JA, Black BS, Johnston D, Hess E, Leoutsakos JM, Gitlin LN et al. A randomized controlled trial of a community-based dementia care coordination intervention: effects of MIND at Home on caregiver outcomes. Am J Geriatr Psychiatry 2015 April;23(4):391-402.

(299) Kales HC, Gitlin LN, Lyketsos CG. Management of neuropsychiatric symptoms of dementia in clinical settings: recommendations from a multidisciplinary expert panel. J Am Geriatr Soc 2014 April;62(4):762-9. 
(300) Teri L, McKenzie G, Coulter CA. Psychosocial interventions of older adults with dementia and their caregivers. In: Schaie W, illis S, editors. Handbook of the psychology of aging. 8th Edition ed. Elselvier: Academic Press.; 2016. p. 447-74.

(301) Selwood A, Johnston K, Katona C, Lyketsos C, Livingston G. Systematic review of the effect of psychological interventions on family caregivers of people with dementia. Journal of Affective Disorders 2007 August;101(1-3):75-89.

(302) Teri L, Huda P, Gibbons L, Young H, van LJ. STAR: a dementia-specific training program for staff in assisted living residences. Gerontologist 2005 October;45(5):686-93.

(303) Mittelman MS, Haley WE, Clay OJ, Roth DL. Improving caregiver well-being delays nursing home placement of patients with Alzheimer disease. Neurology 2006 November $14 ; 67(9): 1592-9$.

(304) Teri L, Gibbons LE, McCurry SM, Logsdon RG, Buchner DM, Barlow WE et al. Exercise plus behavioral management in patients with Alzheimer disease: a randomized controlled trial. JAMA 2003 October 15;290(15):2015-22.

(305) Logsdon RG, Pike KC, McCurry SM, Hunter P, Maher J, Snyder L et al. Early-stage memory loss support groups: outcomes from a randomized controlled clinical trial. J Gerontol B Psychol Sci Soc Sci 2010 November;65(6):691-7.

(306) Hepburn K, Lewis M, Tornatore J, Sherman CW, Bremer KL. The Savvy Caregiver program: the demonstrated effectiveness of a transportable dementia caregiver psychoeducation program. J Gerontol Nurs 2007 March;33(3):30-6.

(307) Gillon R. Ethics needs principles--four can encompass the rest--and respect for autonomy should be "first among equals". J Med Ethics 2003 October;29(5):307-12.

(308) Manthorpe, J. and Moriarty, J. 'Nothing Ventured, Nothing Gained': Risk Guidance for People with Dementia. London: Department of Health; 2010.

(309) Douglas A, Letts L, Richardson J. A systematic review of accidental injury from fire, wandering and medication self-administration errors for older adults with and without dementia. Arch Gerontol Geriatr 2011 January;52(1):e1-10.

(310) Starkstein SE, Jorge R, Mizrahi R, Adrian J, Robinson RG. Insight and danger in Alzheimer's disease. Eur J Neurol 2007 April;14(4):455-60.

(311) Walker AE, Livingston G, Cooper CA, Katona CL, Kitchen GL. Caregivers' experience of risk in dementia: the LASER-AD study. Aging Ment Health 2006 September;10(5):532-8.

(312) Bartus RT, Dean RL, Beer B, Lippa AS. The Cholinergic Hypothesis of Geriatric Memory Dysfunction. Science 1982;217(4558):408-17.

(313) Greenamyre JT, Maragos WF, Albin RL, Penney JB, Young AB. Glutamate Transmission and Toxicity in Alzheimers-Disease. Progress in Neuro-Psychopharmacology \& Biological Psychiatry 1988;12(4):421-30.

(314) Lleo A, Greenberg SM, Growdon JH. Current pharmacotherapy for Alzheimer's disease. Annual Review of Medicine 2006;57:513-33. 
(315) Raschetti R, Albanese E, Vanacore N, Maggini M. Cholinesterase inhibitors in mild cognitive impairment: A systematic review of randomised trials. Plos Medicine 2007 November;4(11):1818-28.

(316) Cooper C, Li R, Lyketsos C, Livingston G. Treatment for mild cognitive impairment: systematic review. British Journal of Psychiatry 2013;203(4):255-64.

(317) Birks J. Cholinesterase inhibitors for Alzheimer's disease. Cochrane Database Syst Rev 2006;(1):CD005593.

(318) Rosen WG, Mohs RC, Davis KL. A new rating scale for Alzheimer's disease. Am J Psychiatry 1984 November;141(11):1356-64.

(319) Howard R, Phillips P, Johnson T, O'Brien J, Sheehan B, Lindesay J et al. Determining the minimum clinically important differences for outcomes in the DOMINO trial. Int J Geriatr Psychiatry 2011 August;26(8):812-7.

(320) Brodaty H, Corey-Bloom J, Potocnik FC, Truyen L, Gold M, Damaraju CR. Galantamine prolonged-release formulation in the treatment of mild to moderate Alzheimer's disease. Dement Geriatr Cogn Disord 2005;20(2-3):120-32.

(321) Feldman HH, Lane R. Rivastigmine: a placebo controlled trial of twice daily and three times daily regimens in patients with Alzheimer's disease. J Neurol Neurosurg Psychiatry 2007 October;78(10):1056-63.

(322) Winblad B, Cummings J, Andreasen N, Grossberg G, Onofrj M, Sadowsky C et al. A sixmonth double-blind, randomized, placebo-controlled study of a transdermal patch in Alzheimer's disease--rivastigmine patch versus capsule. Int J Geriatr Psychiatry 2007 May;22(5):456-67.

(323) Birks J, Grimley EJ, lakovidou V, Tsolaki M, Holt FE. Rivastigmine for Alzheimer's disease. Cochrane Database Syst Rev 2009;(2):CD001191.

(324) Cummings JL, Mega M, Gray K, Rosenberg-Thompson S, Carusi DA, Gornbein J. The Neuropsychiatric Inventory: comprehensive assessment of psychopathology in dementia. Neurology 1994 December;44(12):2308-14.

(325) National Institute for Clinical Excellence. Donepezil, galantamine, rivastigmine and memantine for the treatment of Alzheimer's disease. 2011 Mar 23.

(326) Howard R, McShane R, Lindesay J, Ritchie C, Baldwin A, Barber R et al. Donepezil and memantine for moderate-to-severe Alzheimer's disease. N Engl J Med 2012 March 8;366(10):893-903.

(327) Howard R, McShane R, Lindesay J, Ritchie C, Baldwin A, Barber R et al. Nursing home placement in the Donepezil and Memantine in Moderate to Severe Alzheimer's Disease (DOMINO-AD) trial: secondary and post-hoc analyses. Lancet Neurology 2015 December;14(12):1171-81.

(328) Bohnen NI, Kaufer DI FAU, Hendrickson RF, Ivanco LS FAU, Lopresti BJ FAU, Koeppe RA FAU et al. Degree of inhibition of cortical acetylcholinesterase activity and cognitive effects by donepezil treatment in Alzheimer's disease. J Neurol Neurosurg Psychiatry 2004 May 31;(0022-3050). 
(329) Kuhl DE, Minoshima S FAU - Frey, Frey KA FAU, Foster NL FAU, Kilbourn MR FAU, Koeppe RA. Limited donepezil inhibition of acetylcholinesterase measured with positron emission tomography in living Alzheimer cerebral cortex. Ann Neurol 2000 September 1;(0364-5134 (Print)).

(330) Farlow MR, Salloway S FAU - Tariot P, Tariot PN FAU - Yardley J, Yardley JF, Moline ML FAU - Wang $Q$, Wang $Q F$ et al. Effectiveness and tolerability of high-dose $(23 \mathrm{mg} / \mathrm{d})$ versus standard-dose $(10 \mathrm{mg} / \mathrm{d})$ donepezil in moderate to severe Alzheimer's disease: A 24-week, randomized, double-blind study. Clin Ther 2010 July 1;(1879-114X (Electronic)).

(331) Homma A, Atarashi H, Kubota N, Nakai K, Takase T. Efficacy and Safety of Sustained Release Donepezil High Dose versus Immediate Release Donepezil Standard Dose in Japanese Patients with Severe Alzheimer's Disease: A Randomized, Double-Blind Trial. J Alzheimers Dis 2016 January 24;(1875-8908 (Electronic)).

(332) Cummings JL, Geldmacher DF, Farlow MF, Sabbagh MF, Christensen DF, Betz P. High-dose donepezil ( $23 \mathrm{mg} /$ day) for the treatment of moderate and severe Alzheimer's disease: drug profile and clinical guidelines. CNS Neuroscience \& Therapeutics 2013 January 1;(1755-5949 (Electronic)).

(333) Cummings J, Froelich L, Black SE, Bakchine S, Bellelli G, Molinuevo JL et al. Randomized, double-blind, parallel-group, 48-week study for efficacy and safety of a higher-dose rivastigmine patch (15 vs. $10 \mathrm{~cm}(2)$ ) in Alzheimer's disease. Dement Geriatr Cogn Disord 2012;33(5):341-53.

(334) Alva G, Isaacson R, Sadowsky C, Grossberg G, Meng X, Somogyi M. Efficacy of higher-dose $13.3 \mathrm{mg} / 24 \mathrm{~h}(15 \mathrm{~cm} 2)$ rivastigmine patch on the Alzheimer's Disease Assessment Scalecognitive subscale: domain and individual item analysis. Int J Geriatr Psychiatry 2014 September;29(9):920-7.

(335) McKeith I, Del Ser T, Spano P, Emre M, Wesnes K, Anand R et al. Efficacy of rivastigmine in dementia with Lewy bodies: a randomised, double-blind, placebo-controlled international study. Lancet 2000 December 16;356(9247):2031-6.

(336) Mori E, Ikeda M, Kosaka K, on behalf of the Donepezil-DLB Study Investigators. Donepezil for Dementia with Lewy Bodies: A Randomized, Placebo-Controlled Trial. Ann Neurol 2012 July 23;72:41-52.

(337) Rolinski M, Fox C, Maidment I, McShane R. Cholinesterase inhibitors for dementia with Lewy bodies, Parkinson's disease dementia and cognitive impairment in Parkinson's disease. Cochrane Database Syst Rev 2012;(3):CD006504.

(338) Stinton C, McKeith I, Taylor JP, Lafortune L, Mioshi E, Mak E et al. Pharmacological Management of Lewy Body Dementia: A Systematic Review and Meta-Analysis. Am J Psychiatry 2015 August 1;172(8):731-42.

(339) Kavirajan H, Schneider LS. Efficacy and adverse effects of cholinesterase inhibitors and memantine in vascular dementia: a meta-analysis of randomised controlled trials. Lancet Neurol 2007 September;6(9):782-92.

(340) Li Y, Hai S, Zhou Y, Dong BR. Cholinesterase inhibitors for rarer dementias associated with neurological conditions. Cochrane Database Syst Rev 2015;(3):CD009444. 
(341) Butterfield DA, Pocernich CB. The glutamatergic system and Alzheimer's disease: therapeutic implications. CNS Drugs 2003;17(9):641-52.

(342) McShane R, Sastre AA, Minakaran N. Memantine for dementia. Cochrane Database of Systematic Reviews 2006;(2).

(343) Emre M, Tsolaki M, Bonuccelli U, Dest ${ }^{\circledR}{ }^{\circledR}$ A, Tolosa E, Kutzelnigg A et al. Memantine for patients with Parkinson's disease dementia or dementia with Lewy bodies: a randomised, double-blind, placebo-controlled trial. The Lancet Neurology 2010;9(10):969-77.

(344) Aarsland D, Ballard C, Walker Z, Bostrom F, Alves G, Kossakowski K et al. Memantine in patients with Parkinson's disease dementia or dementia with Lewy bodies: a double-blind, placebo-controlled, multicentre trial. The Lancet Neurology 2009;8(7):613-8.

(345) Schmidt R, Hofer E, Bouwman FH, Buerger K, Cordonnier C, Fladby T et al. EFNS-ENS/EAN Guideline on concomitant use of cholinesterase inhibitors and memantine in moderate to severe Alzheimer's disease. Eur J Neurol 2015 June;22(6):889-98.

(346) Grossberg GT FAU - Farlow M, Farlow MR FAU - Meng X, Meng XF, Velting DM. Evaluating high-dose rivastigmine patch in severe Alzheimer's disease: analyses with concomitant memantine usage as a factor. Curr Alzheimer Res 2015 January 1;(1875-5828 (Electronic)).

(347) Grossberg GT, Manes F, Allegri RF, Gutierrez-Robledo LM, Gloger S, Xie L et al. The safety, tolerability, and efficacy of once-daily memantine $(28 \mathrm{mg})$ : a multinational, randomized, double-blind, placebo-controlled trial in patients with moderate-to-severe Alzheimer's disease taking cholinesterase inhibitors. CNS Drugs 2013 June;27(6):469-78.

(348) de WH, Stam CJ, Lansbergen MM, Wieggers RL, Kamphuis PJ, Scheltens P et al. The effect of souvenaid on functional brain network organisation in patients with mild Alzheimer's disease: a randomised controlled study. PLoS One 2014;9(1):e86558.

(349) Rijpma A, Meulenbroek O, van Hees AM, Sijben JW, Vellas B, Shah RC et al. Effects of Souvenaid on plasma micronutrient levels and fatty acid profiles in mild and mild-tomoderate Alzheimer's disease. Alzheimers Res Ther 2015;7(1):51.

(350) Shah RC, Kamphuis PJ, Leurgans S, Swinkels SH, Sadowsky CH, Bongers A et al. The SConnect study: results from a randomized, controlled trial of Souvenaid in mild-tomoderate Alzheimer's disease. Alzheimers Res Ther 2013;5(6):59.

(351) Onakpoya IJ, Heneghan CJ. The efficacy of supplementation with the novel medical food, Souvenaid, in patients with Alzheimer's disease: A systematic review and meta-analysis of randomized clinical trials. Nutr Neurosci 2015 December 7.

(352) Clare L, Woods R. Cognitive training and cognitive rehabilitation for people with earlystage Alzheimer's disease: A review. Neuropsychological Rehabilitation 2004 September 1;14(4):385-401.

(353) Clare L, Bayer A, Burns A, Corbett A, Jones R, Knapp M et al. Goal-oriented cognitive rehabilitation in early-stage dementia: study protocol for a multi-centre single-blind randomised controlled trial (GREAT). Trials 2013;14:152. 
(354) Spector A, Thorgrimsen L, Woods B, Royan L, Davies S, Butterworth M et al. Efficacy of an evidence-based cognitive stimulation therapy programme for people with dementia: randomised controlled trial. Br J Psychiatry 2003 September;183:248-54.

(355) Huntley JD, Gould RL, Liu K, Smith M, Howard RJ. Do cognitive interventions improve general cognition in dementia? A meta-analysis and meta-regression. BMJ Open 2015;5(4):e005247.

(356) Woods B, Aguirre E, Spector AE, Orrell M. Cognitive stimulation to improve cognitive functioning in people with dementia. Cochrane Database Syst Rev 2012;2.

(357) Orgeta V, Leung P, Yates L, Kang S, Hoare Z, Henderson C et al. Individual cognitive stimulation therapy for dementia: a clinical effectiveness and cost-effectiveness pragmatic, multicentre, randomised controlled trial. Health Technol Assess 2015 August;19(64):1-108.

(358) Bahar-Fuchs A, Clare L, Woods B. Cognitive training and cognitive rehabilitation for mild to moderate AlzheimerГÇÖs disease and vascular dementia. Cochrane Database Syst Rev $2013 ; 6$.

(359) Huntley JD, Hampshire A, Bor D, Owen A, Howard RJ. Adaptive working memory strategy training in early Alzheimer's disease: randomised controlled trial. Br J Psychiatry 2017 January;210(1):61-6.

(360) Amieva H, Robert PH, Grandoulier AS, Meillon C, De RJ, Andrieu S et al. Group and individual cognitive therapies in Alzheimer's disease: the ETNA3 randomized trial. Int Psychogeriatr 2016 May;28(5):707-17.

(361) Farina N, Rusted J, Tabet N. The effect of exercise interventions on cognitive outcome in Alzheimer's disease: a systematic review. Int Psychogeriatr 2014 January;26(1):9-18.

(362) Forbes D, Forbes SC, Blake CM, Thiessen EJ, Forbes S. Exercise programs for people with dementia. Cochrane Database Syst Rev 2015;4:CD006489.

(363) Ohman H, Savikko N, Strandberg TE, Kautiainen H, Raivio MM, Laakkonen ML et al. Effects of Exercise on Cognition: The Finnish Alzheimer Disease Exercise Trial: A Randomized, Controlled Trial. J Am Geriatr Soc 2016 April;64(4):731-8.

(364) Pitkala KH, Poysti MM, Laakkonen ML, Tilvis RS, Savikko N, Kautiainen $\mathrm{H}$ et al. Effects of the Finnish Alzheimer disease exercise trial (FINALEX): a randomized controlled trial. JAMA Intern Med 2013 May 27;173(10):894-901.

(365) Yu F, Bronas UG, Konety S, Nelson NW, Dysken M, Jack C, Jr. et al. Effects of aerobic exercise on cognition and hippocampal volume in Alzheimer's disease: study protocol of a randomized controlled trial (The FIT-AD trial). Trials 2014;15:394.

(366) Angevaren M, Vanhees L, Wendel-Vos W, Verhaar HJ, Aufdemkampe G, Aleman A et al. Intensity, but not duration, of physical activities is related to cognitive function. Eur J Cardiovasc Prev Rehabil 2007 December;14(6):825-30.

(367) Hoffmann K, Frederiksen KS, Sobol NA, Beyer N, Vogel A, Simonsen AH et al. Preserving cognition, quality of life, physical health and functional ability in Alzheimer's disease: the effect of physical exercise (ADEX trial): rationale and design. Neuroepidemiology 2013;41(3-4):198-207. 
(368) Hoffmann K, Sobol NA, Frederiksen KS, Beyer N, Vogel A, Vestergaard K et al. Moderate-toHigh Intensity Physical Exercise in Patients with Alzheimer's Disease: A Randomized Controlled Trial. J Alzheimers Dis 2015 December 10;50(2):443-53.

(369) Savva GM, Zaccai J, Matthews FE, Davidson JE, McKeith I, Brayne C. Prevalence, correlates and course of behavioural and psychological symptoms of dementia in the population. $\mathrm{Br} \mathrm{J}$ Psychiatry 2009 March;194(3):212-9.

(370) Lyketsos CG, Lopez O, Jones B, Fitzpatrick AL, Breitner J, DeKosky S. Prevalence of neuropsychiatric symptoms in dementia and mild cognitive impairment: results from the cardiovascular health study. JAMA 2002 September 25;288(12):1475-83.

(371) Lyketsos CG, Sheppard JM, Steinberg M, Tschanz JA, Norton MC, Steffens DC et al. Neuropsychiatric disturbance in Alzheimer's disease clusters into three groups: the Cache County study. Int J Geriatr Psychiatry 2001 November;16(11):1043-53.

(372) Ballard C, Aarsland D, Francis P, Corbett A. Neuropsychiatric symptoms in patients with dementias associated with cortical Lewy bodies: pathophysiology, clinical features, and pharmacological management. Drugs Aging 2013 August;30(8):603-11.

(373) Steinberg M, Tschanz JT, Corcoran C, Steffens DC, Norton MC, Lyketsos CG et al. The persistence of neuropsychiatric symptoms in dementia: the Cache County Study. Int J Geriatr Psychiatry 2004 January;19(1):19-26.

(374) van der Linde RM, Dening T, Stephan BC, Prina AM, Evans E, Brayne C. Longitudinal course of behavioural and psychological symptoms of dementia: systematic review. The British Journal of Psychiatry 2016;bjp-bp.

(375) Aalten P, Verhey FR FAU - Boziki M, Boziki MF, Bullock RF, Byrne EJ FAU - Camus V, Camus $V F$ et al. Neuropsychiatric syndromes in dementia. Results from the European Alzheimer Disease Consortium: part. Dement Geriatr Cogn Disord 2007 January 1;24(1421-9824 (Electronic)):457-763.

(376) Kales HC, Gitlin LN, Lyketsos CG. Assessment and management of behavioral and psychological symptoms of dementia. Bmj-British Medical Journal 2015 March 2;350.

(377) Ryu SH, Katona C, Rive B, Livingston G. Persistence of and changes in neuropsychiatric symptoms in Alzheimer disease over 6 months: the LASER-AD study. Am J Geriatr Psychiatry 2005 November;13(11):976-83.

(378) Zahodne LB, Ornstein K, Cosentino S, Devanand DP, Stern Y. Longitudinal Relationships Between Alzheimer Disease Progression and Psychosis, Depressed Mood, and Agitation/Aggression. American Journal of Geriatric Psychiatry 2015 February;23(2):13040.

(379) Stern Y, Mayeux R, Sano M, Hauser WA, Bush T. Predictors of disease course in patients with probable Alzheimer's disease. Neurology 1987 October;37(10):1649-53.

(380) Chui HC, Lyness SA, Sobel E, Schneider LS. Extrapyramidal signs and psychiatric symptoms predict faster cognitive decline in Alzheimer's disease. Arch Neurol 1994 July;51(7):676-81. 
(381) Livingston G, Walker AE, Katona CL, Cooper C. Antipsychotics and cognitive decline in Alzheimer's disease: the LASER-Alzheimer's disease longitudinal study. J Neurol Neurosurg Psychiatry 2007 January; 78(1):25-9.

(382) Mendez MF, Shapira JS, Woods RJ, Licht EA, Saul RE. Psychotic symptoms in frontotemporal dementia: Prevalence and review. Dementia and Geriatric Cognitive Disorders 2008;25(3):206-11.

(383) Snowden JS, Rollinson S, Thompson JC, Harris JM, Stopford CL, Richardson AM et al. Distinct clinical and pathological characteristics of frontotemporal dementia associated with C9ORF72 mutations. Brain 2012 March;135(Pt 3):693-708.

(384) Murray PS, Kumar S, DeMichele-Sweet MAA, Sweet RA. Psychosis in Alzheimer's Disease. Biological Psychiatry 2014 April 1;75(7):542-52.

(385) Cohen-Mansfield J. Nonpharmacologic interventions for psychotic symptoms in dementia. Journal of Geriatric Psychiatry and Neurology 2003 December;16(4):219-24.

(386) Cohen-Mansfield J, Cohen R, Golander H, Heinik J. The impact of psychotic symptoms on the persons with dementia experiencing them. American Journal of Geriatric Psychiatry 2016 March;24(3):213-20.

(387) Matsunaga S, Kishi T, Yasue I, Iwata N. Cholinesterase Inhibitors for Lewy Body Disorders: A Meta-Analysis. Int J Neuropsychopharmacol 2016 February 28;19(2):yv086.

(388) Maust DT, Kim HM, Seyfried LS, Chiang C, Kavanagh J, Schneider LS et al. Antipsychotics, Other Psychotropics, and the Risk of Death in Patients With Dementia Number Needed to Harm. Jama Psychiatry 2015 May;72(5):438-45.

(389) Schneider LS, Dagerman K, Insel PS. Efficacy and adverse effects of atypical antipsychotics for dementia: Meta-analysis of randomized, placebo-controlled trials. American Journal of Geriatric Psychiatry 2006 March;14(3):191-210.

(390) Kales HC, Valenstein M, Kim HM, McCarthy JF, Ganoczy D, Cunningham F et al. Mortality risk in patients with dementia treated with antipsychotics versus other psychiatric medications. Am J Psychiatry 2007 October;164(10):1568-76.

(391) Kales HC, Zivin K, Kim HM, Valenstein M, Chiang C, Ignacio RV et al. Trends in antipsychotic use in dementia 1999-2007. Arch Gen Psychiatry 2011 February;68(2):190-7.

(392) Wang PS, Schneeweiss S, Avorn J, Fischer MA, Mogun H, Solomon DH et al. Risk of death in elderly users of conventional vs. atypical antipsychotic medications. N Engl J Med 2005 December 1;353(22):2335-41.

(393) Huybrechts KF, Gerhard T, Crystal S, Olfson M, Avorn J, Levin R et al. Differential risk of death in older residents in nursing homes prescribed specific antipsychotic drugs: population based cohort study. BMJ 2012;344:e977.

(394) Kales HC, Kim HM, Zivin K, Valenstein M, Seyfried LS, Chiang C et al. Risk of mortality among individual antipsychotics in patients with dementia. Am J Psychiatry 2012 January;169(1):71-9. 
(395) Arai H, Nakamura Y, Taguchi M, Kobayashi H, Yamauchi K, Schneider LS. Mortality risk in current and new antipsychotic AD users: Large scale Japanese study. Alzheimers Dement 2016 April 19.

(396) Banerjee $\mathrm{S}$. The use of antipsychotic medication for people with dementia:

Time for action A report . Department of Health 2010;Available from: URL: http://www.dh.gov.uk/prod consum dh/groups/dh digitalassets/documents/digitalasset Ldh 108302.pdf

(397) Health and social care information centre. National Dementia \& Antipsychotic Prescribing Audit: Key findings on the prescription of antipsychotics for people with dementia in England. Report for the audit period 2006 to 2011. 2012 Jul 17.

(398) Howard R, Costafreda S, Karcher K, Coppolla D, Berlin J, Hough D. Baseline characteristics and treatment-emergent risk factors associated with cerebrovascular event and death with risperidone in dementia patients. Br J Psychiatry 2016.

(399) Vigen CL, Mack WJ, Keefe RS, Sano M, Sultzer DL, Stroup TS et al. Cognitive effects of atypical antipsychotic medications in patients with Alzheimer's disease: outcomes from CATIE-AD. Am J Psychiatry 2011 August;168(8):831-9.

(400) Schneider LS, Dagerman K, Insel PS. Efficacy and adverse effects of atypical antipsychotics for dementia: meta-analysis of randomized, placebo-controlled trials. Am J Geriatr Psychiatry 2006 March;14(3):191-210.

(401) Schneider LS, Tariot PN, Dagerman KS, Davis SM, Hsiao JK, Ismail MS et al. Effectiveness of atypical antipsychotic drugs in patients with Alzheimer's disease. N Engl J Med 2006 October 12;355(15):1525-38.

(402) Lee PE, Gill SS, Freedman M, Bronskill SE, Hillmer MP, Rochon PA. Atypical antipsychotic drugs in the treatment of behavioural and psychological symptoms of dementia: systematic review. BMJ 2004 July 10;329(7457):75.

(403) Sultzer DL, Davis SM, Tariot PN, Dagerman KS, Lebowitz BD, Lyketsos CG et al. Clinical symptom responses to atypical antipsychotic medications in Alzheimer's disease: phase 1 outcomes from the CATIE-AD effectiveness trial. Am J Psychiatry 2008 July;165(7):844-54.

(404) Ballard C, Margallo-Lana M, Juszczak E, Douglas S, Swann A, Thomas A et al. Quetiapine and rivastigmine and cognitive decline in Alzheimer's disease: randomised double blind placebo controlled trial. BMJ 2005 April 16;330(7496):874.

(405) Ballard C, Lana MM, Theodoulou M, Douglas S, McShane R, Jacoby R et al. A randomised, blinded, placebo-controlled trial in dementia patients continuing or stopping neuroleptics (the DART-AD trial). PLoS Med 2008 April 1;5(4):e76.

(406) Devanand DP, Mintzer J, Schultz SK, Andrews HF, Sultzer DL, de la Pena D et al. Relapse risk after discontinuation of risperidone in Alzheimer's disease. N Engl J Med 2012 October 18;367(16):1497-507.

(407) Ballard C, Thomas A, Gerry S, Yu LM, Aarsland D, Merritt C et al. A double-blind randomized placebo-controlled withdrawal trial comparing memantine and antipsychotics for the long-term treatment of function and neuropsychiatric symptoms in people with Alzheimer's disease (MAIN-AD). J Am Med Dir Assoc 2015 April;16(4):316-22. 
(408) Cummings J, Mintzer J, Brodaty H, Sano M, Banerjee S, Devanand DP et al. Agitation in cognitive disorders: International Psychogeriatric Association provisional consensus clinical and research definition. Int Psychogeriatr 2015 January;27(1):7-17.

(409) Cohen-Mansfield J. Conceptualization of agitation: results based on the Cohen-Mansfield Agitation Inventory and the Agitation Behavior Mapping Instrument. Int Psychogeriatr 1996;8 Suppl 3:309-15.

(410) Livingston G, Kelly L, Lewis-Holmes E, Baio G, Morris S, Patel N et al. A systematic review of the clinical effectiveness and cost-effectiveness of sensory, psychological and behavioural interventions for managing agitation in older adults with dementia. Health technology assessment (Winchester, England) 2014;18(39):1-226.

(411) Rosenberg PB, Nowrangi MA, Lyketsos CG. Neuropsychiatric symptoms in Alzheimer's disease: What might be associated brain circuits? Molecular Aspects of Medicine 2015 June;43-44:25-37.

(412) Morris S, Patel N, Baio G, Kelly L, Lewis-Holmes E, Omar RZ et al. Monetary costs of agitation in older adults with Alzheimer's disease in the UK: prospective cohort study. BMJ Open 2015;5(3):e007382.

(413) Livingston G, Kelly L, Lewis-Holmes E, Baio G, Morris S, Patel N et al. Non-pharmacological interventions for agitation in dementia: systematic review of randomised controlled trials. Br J Psychiatry 2014 December;205(6):436-42.

(414) Teri L, Logsdon RG, McCurry SM. Exercise interventions for dementia and cognitive impairment: the Seattle Protocols. J Nutr Health Aging 2008 June;12(6):391-4.

(415) Jutkowitz E, Gitlin L, Pizzi LT. Evaluating Willingness to Pay Thresholds for A Dementia Caregiving Intervention. Value in Health 2010 May;13(3):A13.

(416) Cooper C, Mukadam N, Katona C, Lyketsos CG, Blazer D, Ames D et al. Systematic Review of the Effectiveness of Pharmacologic Interventions to Improve Quality of Life and Wellbeing in People With Dementia. Am J Geriatr Psychiatry 2012 January 12.

(417) Farina N, Rusted J, Tabet $\mathrm{N}$. The effect of exercise interventions on cognitive outcome in Alzheimer's disease: a systematic review. Int Psychogeriatr 2014 January;26(1):9-18.

(418) Carlson MC, Saczynski JS, Rebok GW, Seeman T, Glass TA, McGill S et al. Exploring the effects of an "everyday" activity program on executive function and memory in older adults: Experience Corps. Gerontologist 2008 December;48(6):793-801.

(419) Cohen-Mansfield J, Marx MS, Dakheel-Ali M, Regier NG, Thein K, Freedman L. Can agitated behavior of nursing home residents with dementia be prevented with the use of standardized stimuli? J Am Geriatr Soc 2010 August;58(8):1459-64.

(420) Logsdon RG, Teri L. The Pleasant Events Schedule-AD: psychometric properties and relationship to depression and cognition in Alzheimer's disease patients. Gerontologist 1997 February;37(1):40-5.

(421) Regier NG, Hodgson N, Gitlin L.

Characteristics of Activities for Persons with Dementia at the Mild, Moderate and Severe Stages . The Gerontologist, Practice Concepts. In press 2016. 
(422) Cooper C, Mukadam N, Katona C, Lyketsos CG, Ames D, Rabins P et al. Systematic review of the effectiveness of non-pharmacological interventions to improve quality of life of people with dementia. International Psychogeriatrics 2012 June;24(6):856-70.

(423) Cohen-Mansfield J, Creedon MA, Malone T, Parpura-Gill A, Dakheel-Ali M, Heasly C. Dressing of cognitively impaired nursing home residents: description and analysis. Gerontologist 2006 February;46(1):89-96.

(424) The MARQUE project: Managing Agitation and Raising QUality of Life: Cluster RCT to improve agitation for people with dementia in care homes. 2016. ISCTRN registry. 28-52016.

Ref Type: Online Source

(425) Cohen-Mansfield J, Marx MS, Dakheel-Ali M, Regier NG, Thein K. Can persons with dementia be engaged with stimuli? Am J Geriatr Psychiatry 2010 April;18(4):351-62.

(426) Cohen-Mansfield J, Werner P. Management of verbally disruptive behaviors in nursing home residents. J Gerontol A Biol Sci Med Sci 1997 November;52(6):M369-M377.

(427) Cohen-Mansfield J, Marx MS, Thein K, Dakheel-Ali M. The impact of stimuli on affect in persons with dementia. J Clin Psychiatry 2011 April;72(4):480-6.

(428) Ballard C, Brown R, Fossey J, Douglas S, Bradley P, Hancock J et al. Brief psychosocial therapy for the treatment of agitation in Alzheimer disease (the CALM-AD trial). Am J Geriatr Psychiatry 2009 September;17(9):726-33.

(429) Livingston G, Kelly L, Lewis-Holmes E, Baio G, Morris S, Patel N et al. A systematic review of the clinical effectiveness and cost-effectiveness of sensory, psychological and behavioural interventions for managing agitation in older adults with dementia. Health Technol Assess 2014 June;18(39):1-vi.

(430) Cohen-Mansfield J. Nonpharmacological Management of Behavioral Problems in Persons with Dementia: The TREA Model. Alzheimer's Care Today 2000;1(4).

(431) Ballard C, Howard R. Neuroleptic drugs in dementia: benefits and harm. Nat Rev Neurosci 2006 June;7(6):492-500.

(432) Cohen-Mansfield J, Lipson S, Werner P, Billig N, Taylor L, Woosley R. Withdrawal of haloperidol, thioridazine, and lorazepam in the nursing home: a controlled, double-blind study. Arch Intern Med 1999 August 9;159(15):1733-40.

(433) Fox C, Crugel M, Maidment I, Auestad BH, Coulton S, Treloar A et al. Efficacy of Memantine for Agitation in Alzheimer's Dementia: A Randomised Double-Blind Placebo Controlled Trial. Plos One 2012;7(5).

(434) Howard RJ, Juszczak E, Ballard CG, Bentham P, Brown RG, Bullock R et al. Donepezil for the treatment of agitation in Alzheimer's disease. New England Journal of Medicine 2007 October 4;357(14):1382-92.

(435) Ballard C, Thomas A, Gerry S, Yu LM, Aarsland D, Merritt C et al. A double-blind randomized placebo-controlled withdrawal trial comparing memantine and antipsychotics for the long-term treatment of function and neuropsychiatric symptoms in people with Alzheimer's disease (MAIN-AD). J Am Med Dir Assoc 2015 April;16(4):316-22. 
(436) Drye LT, Spragg D, Devanand DP, Frangakis C, Marano C, Meinert CL et al. Changes in QTC interval in the citalopram for agitation in Alzheimer's disease (CitAD) randomized trial. Plos One 2014;9(6):e98426.

(437) Porsteinsson AP, Drye LT, Pollock BG, Devanand DP, Frangakis C, Ismail Z et al. Effect of citalopram on agitation in Alzheimer disease: the CitAD randomized clinical trial. JAMA 2014 February 19;311(7):682-91.

(438) Weintraub D, Drye LT, Porsteinsson AP, Rosenberg PB, Pollock BG, Devanand DP et al. Time to Response to Citalopram Treatment for Agitation in Alzheimer Disease. Am J Geriatr Psychiatry 2015 November;23(11):1127-33.

(439) Ho T, Pollock BG, Mulsant BH, Schantz O, Devanand DP, Mintzer JE et al. R- and Scitalopram concentrations have differential effects on neuropsychiatric scores in elders with dementia and agitation. Br J Clin Pharmacol 2016 September;82(3):784-92.

(440) Hedenmalm K, Guzey C, Dahl ML, Yue QY, Spigset O. Risk factors for extrapyramidal symptoms during treatment with selective serotonin reuptake inhibitors, including cytochrome P-450 enzyme, and serotonin and dopamine transporter and receptor polymorphisms. J Clin Psychopharmacol 2006 April;26(2):192-7.

(441) Schneider LS, Frangakis C, Drye LT, Devanand DP, Marano CM, Mintzer J et al. Heterogeneity of Treatment Response to Citalopram for Patients With Alzheimer's Disease With Aggression or Agitation: The CitAD Randomized Clinical Trial. American Journal of Psychiatry 2016 May;173(5):465-72.

(442) Street JS, Clark WS, Gannon KS, Cummings JL, Bymaster FP, Tamura RN et al. Olanzapine treatment of psychotic and behavioral symptoms in patients with Alzheimer disease in nursing care facilities: a double-blind, randomized, placebo-controlled trial. The HGEU Study Group. Arch Gen Psychiatry 2000 October;57(10):968-76.

(443) US Food and Drug Administration (FDA). Revised recommendations for Celexa (citalopram hydrobromide) related to a potential risk of abnormal heart rhythms with high doses. 18-32012. Silver Springs Md, FDA.

Ref Type: Online Source

(444) Pollock BG, Mulsant BH, Rosen J, Mazumdar S, Blakesley RE, Houck PR et al. A double-blind comparison of citalopram and risperidone for the treatment of behavioral and psychotic symptoms associated with dementia. Am J Geriatr Psychiatry 2007 November;15(11):94252.

(445) Pollock BG, Mulsant BH, Rosen J, Sweet RA, Mazumdar S, Bharucha A et al. Comparison of citalopram, perphenazine, and placebo for the acute treatment of psychosis and behavioral disturbances in hospitalized, demented patients. Am J Psychiatry 2002 March;159(3):460-5.

(446) Cummings JL, Lyketsos CG, Peskind ER, Porsteinsson AP, Mintzer JE, Scharre DW et al. Effect of Dextromethorphan-Quinidine on Agitation in Patients With Alzheimer Disease Dementia: A Randomized Clinical Trial. JAMA 2015 September 22;314(12):1242-54.

(447) Husebo BS, Ballard C, Sandvik R, Nilsen OB, Aarsland D. Efficacy of treating pain to reduce behavioural disturbances in residents of nursing homes with dementia: cluster randomised clinical trial. BMJ 2011;343:d4065. 
(448) Coupland C, Dhiman P, Morriss R, Arthur A, Barton G, Hippisley-Cox J. Antidepressant use and risk of adverse outcomes in older people: population based cohort study. BMJ 2011 August 2;343:d4551.

(449) Ballard CG, Bannister C, Oyebode F. Depression in dementia sufferers. Int J Geriatr Psychiatry 1996 June;11(6):507-15.

(450) Burns A. Affective Symptoms in Alzheimers-Disease. Int J Geriatr Psychiatry 1991 June;6(6):371-6.

(451) Greenwald BS, Kramerginsberg E, Marin DB, Laitman LB, Hermann CK, Mohs RC et al. Dementia with Coexistent Major Depression. American Journal of Psychiatry 1989 November;146(11):1472-8.

(452) Banerjee S, Hellier J, Dewey M, Romeo R, Ballard C, Baldwin R et al. Sertraline or mirtazapine for depression in dementia (HTA-SADD): a randomised, multicentre, doubleblind, placebo-controlled trial. Lancet 2011 July 30;378(9789):403-11.

(453) Zubenko GS, Zubenko WN, McPherson S, Spoor E, Marin DB, Farlow MR et al. A collaborative study of the emergence and clinical features of the major depressive syndrome of Alzheimer's disease. Am J Psychiatry 2003 May;160(5):857-66.

(454) Farina N, Morel L, Banerjee S. What is the therapeutic value of antidepressants in dementia? A narrative review. Int J Geriatr Psychiatry 2016.

(455) Zubenko GS, Moossy J. Major depression in primary dementia. Clinical and neuropathologic correlates. Arch Neurol 1988 November;45(11):1182-6.

(456) Orgeta V, Qazi A, Spector AE, Orrell M. Psychological treatments for depression and anxiety in dementia and mild cognitive impairment. Cochrane Database Syst Rev 2014;(1):CD009125.

(457) Orgeta V, Qazi A, Spector A, Orrell M. Psychological treatments for depression and anxiety in dementia and mild cognitive impairment: systematic review and meta-analysis. $\mathrm{Br} \mathrm{J}$ Psychiatry 2015 October;207(4):293-8.

(458) Nelson JC, Devanand DP. A Systematic Review and Meta-Analysis of Placebo-Controlled Antidepressant Studies in People with Depression and Dementia. Journal of the American Geriatrics Society 2011 April;59(4):577-85.

(459) Laitinen ML, Lonnroos E, Bell JS, Lavikainen P, Sulkava R, Hartikainen S. Use of antidepressants among community-dwelling persons with Alzheimer's disease: a nationwide register-based study. International Psychogeriatrics 2015 April;27(4):669-72.

(460) Bains J, Birks J, Dening T. Antidepressants for treating depression in dementia. Cochrane Database Syst Rev 2002;(4):CD003944.

(461) Drye LT, Martin BK, Frangakis CE, Meinert CL, Mintzer JE, Munro CA et al. Do treatment effects vary among differing baseline depression criteria in depression in Alzheimer's disease study +/- 2 (DIADS-2)? Int J Geriatr Psychiatry 2011 June;26(6):573-83. 
(462) Rosenberg PB, Drye LT, Martin BK, Frangakis C, Mintzer JE, Weintraub D et al. Sertraline for the treatment of depression in Alzheimer disease. Am J Geriatr Psychiatry 2010 February;18(2):136-45.

(463) Weintraub D, Rosenberg PB, Drye LT, Martin BK, Frangakis C, Mintzer JE et al. Sertraline for the treatment of depression in Alzheimer disease: week-24 outcomes. Am J Geriatr Psychiatry 2010 April;18(4):332-40.

(464) Roth M, Mountjoy CQ, Amrein R. Moclobemide in elderly patients with cognitive decline and depression: an international double-blind, placebo-controlled trial. Br J Psychiatry 1996 February;168(2):149-57.

(465) Bergh S, Selbaek G, Engedal K. Discontinuation of antidepressants in people with dementia and neuropsychiatric symptoms (DESEP study): double blind, randomised, parallel group, placebo controlled trial. BMJ 2012;344:e1566.

(466) Moran M, Lynch CA, Walsh C, Coen R, Coakley D, Lawlor BA. Sleep disturbance in mild to moderate Alzheimer's disease. Sleep Medicine 2005 July;6(4):347-52.

(467) Dauvilliers Y. Insomnia in patients with neurodegenerative conditions. Sleep Medicine 2007 December;8:S27-S34.

(468) Chwiszczuk L, Breitve M, Hynninen M, Gjerstad MD, Aarsland D, Rongve A. Higher Frequency and Complexity of Sleep Disturbances in Dementia with Lewy Bodies as Compared to Alzheimer's Disease. Neurodegener Dis 2016;16(3-4):152-60.

(469) Ju YES, Lucey BP, Holtzman DM. Sleep and Alzheimer disease pathology-a bidirectional relationship. Nature Reviews Neurology 2014 February;10(2):115-9.

(470) van Someren EJ, Hagebeuk EE, Lijzenga C, Scheltens P, de Rooij SE, Jonker C et al. Circadian rest-activity rhythm disturbances in Alzheimer's disease. Biol Psychiatry 1996 August $15 ; 40(4): 259-70$.

(471) McCrae CS, Dzierzewski JM, McNamara JP, Vatthauer KE, Roth AJ, Rowe MA. Changes in Sleep Predict Changes in Affect in Older Caregivers of Individuals with Alzheimer's Dementia: A Multilevel Model Approach. J Gerontol B Psychol Sci Soc Sci 2014 November 26.

(472) McCleery J, Cohen D, Sharpley A. Pharmacotherapies for sleep disturbances in Alzheimer's disease. Cochrane Databaseof Systematic Reviews 2014;(3).

(473) Forbes D, Blake C, Thiessen E, Peacock S, Hawranik P. Light therapy for improving cognition, activities of daily living, sleep, challenging behaviour, and psychiatric disturbances in dementia. Cochrane Database of Systematic Reviews 2014;CD003946. DOI: 10.1002/14651858.CD003946.pub4.

(474) Brown CA, Berry R, Tan MC, Khoshia A, Turlapati L, Swedlove F. A critique of the evidence base for non-pharmacological sleep interventions for persons with dementia. Dementia (London) 2013 March;12(2):210-37.

(475) McCurry SM, Gibbons LE, Logsdon RG, Vitiello MV, Teri L. Nighttime insomnia treatment and education for Alzheimer's disease: a randomized, controlled trial. J Am Geriatr Soc 2005 May;53(5):793-802. 
(476) Bourgeois J, Elseviers MM, Van BL, Petrovic M, Vander Stichele RH. One-year evolution of sleep quality in older users of benzodiazepines: a longitudinal cohort study in belgian nursing home residents. Drugs Aging 2014 September;31(9):677-82.

(477) Berry SD, Placide SG, Mostofsky E, Zhang Y, Lipsitz LA, Mittleman MA et al. Antipsychotic and Benzodiazepine Drug Changes Affect Acute Falls Risk Differently in the Nursing Home. J Gerontol A Biol Sci Med Sci 2016 February;71(2):273-8.

(478) Weich S, Pearce HL, Croft P, Singh S, Crome I, Bashford J et al. Effect of anxiolytic and hypnotic drug prescriptions on mortality hazards: retrospective cohort study. BMJ 2014;348:1996.

(479) Howell MJ, Schenck CH. Rapid Eye Movement Sleep Behavior Disorder and Neurodegenerative Disease. JAMA Neurol 2015 June;72(6):707-12.

(480) Jiang H, Huang J, Shen Y, Guo S, Wang L, Han C et al. RBD and Neurodegenerative Diseases. Mol Neurobiol 2016 March 31.

(481) Brodaty H, Burns K. Nonpharmacological management of apathy in dementia: a systematic review. Am J Geriatr Psychiatry 2012 July;20(7):549-64.

(482) Rajkumar AP, Ballard C, Fossey J, Corbett A, Woods B, Orrell M et al. Apathy and Its Response to Antipsychotic Review and Nonpharmacological Interventions in People With Dementia Living in Nursing Homes: WHELD, a Factorial Cluster Randomized Controlled Trial. J Am Med Dir Assoc 2016 May 12.

(483) Rosenberg PB, Lanctot KL, Drye LT, Herrmann N, Scherer RW, Bachman DL et al. Safety and efficacy of methylphenidate for apathy in Alzheimer's disease: a randomized, placebocontrolled trial. J Clin Psychiatry 2013 August;74(8):810-6.

(484) Samsi K, Manthorpe J. Everyday decision-making in dementia: findings from a longitudinal interview study of people with dementia and family carers. Int Psychogeriatr 2013 June;25(6):949-61.

(485) Karlawish JH. Living with dementia: caregiver perspectives. LDI Issue Brief 2002 June;7(8):1-4.

(486) Lord K, Livingston G, Cooper C. A systematic review of barriers and facilitators to and interventions for proxy decision-making by family carers of people with dementia. Int Psychogeriatr 2015 August;27(8):1301-12.

(487) Butcher HK, Holkup PA, Park M, Maas M. Thematic analysis of the experience of making a decision to place a family member with Alzheimer's disease in a special care unit. Res Nurs Health 2001 December;24(6):470-80.

(488) Hirschman KB, Kapo JM, Karlawish JH. Why doesn't a family member of a person with advanced dementia use a substituted judgment when making a decision for that person? Am J Geriatr Psychiatry 2006 August;14(8):659-67.

(489) Mezey M, Kluger M, Maislin G, Mittelman M. Life-sustaining treatment decisions by spouses of patients with Alzheimer's disease. J Am Geriatr Soc 1996 February;44(2):14450. 
(490) Lord K, Livingston G, Robertson S, Cooper C. How people with dementia and their families decide about moving to a care home and support their needs: development of a decision aid, a qualitative study. BMC Geriatr 2016;16(1):68.

(491) Lord K, Livingston G, Cooper C. A feasibility Randomised Controlled Trial of the Decide intervention: Dementia Carers Making Informed Decisions . British Journal Psychiatry open. In press 2016.

(492) Cooper C, Balamurali TB, Livingston G. A systematic review of the prevalence and covariates of anxiety in caregivers of people with dementia. Int Psychogeriatr 2007 April;19(2):175-95.

(493) Goren A, Montgomery W, Kahle-Wrobleski K, Nakamura T, Ueda K. Impact of caring for persons with Alzheimer's disease or dementia on caregivers' health outcomes: findings from a community based survey in Japan. BMC Geriatr 2016;16(1):122.

(494) Norton MC, Smith KR, Ostbye T, Tschanz JT, Corcoran C, Schwartz S et al. Greater risk of dementia when spouse has dementia? The Cache County study. J Am Geriatr Soc 2010 May;58(5):895-900.

(495) Cooper C, Balamurali TB, Selwood A, Livingston G. A systematic review of intervention studies about anxiety in caregivers of people with dementia. Int J Geriatr Psychiatry 2007 March;22(3):181-8.

(496) Gallagher D, Ni MA, Crosby L, Ryan D, Lacey L, Coen RF et al. Determinants of the desire to institutionalize in Alzheimer's caregivers. Am J Alzheimers Dis Other Demen 2011 May;26(3):205-11.

(497) Cooper C, Selwood A, Blanchard M, Walker Z, Blizard R, Livingston G. The determinants of family carers' abusive behaviour to people with dementia: Results of the CARD study. Journal of Affective Disorders 2010 February;121(1-2):136-42.

(498) Hoe J, Katona C, Orrell M, Livingston G. Quality of life in dementia: care recipient and caregiver perceptions of quality of life in dementia: the LASER-AD study. Int J Geriatr Psychiatry 2007 October;22(10):1031-6.

(499) Spijker, A, Vernooij-Dassen M, Vasse E. Effectiveness of nonpharmacological interventions in delaying the institutionalization of patients with dementia: a meta-analysis. Journal of the American Geriatrics Society 56, 1116-1128. 2008.

Ref Type: Generic

(500) Pinquart M, Sorensen S. Helping caregivers of persons with dementia: which interventions work and how large are their effects? International Psychogeriatrics 2006 December;18(4):577-95.

(501) Gitlin LN, Belle SH, Burgio LD, Czaja SJ, Mahoney D, Gallagher-Thompson D et al. Effect of multicomponent interventions on caregiver burden and depression: the REACH multisite initiative at 6-month follow-up. Psychol Aging 2003 September;18(3):361-74.

(502) Teri L, McCurry SM, Logsdon R, Gibbons LE. Training community consultants to help family members improve dementia care: a randomized controlled trial. Gerontologist 2005 December;45(6):802-11. 
(503) Li R, Cooper C, Bradley J, Shulman A, Livingston G. Coping strategies and psychological morbidity in family carers of people with dementia: a systematic review and metaanalysis. J Affect Disord 2012 June;139(1):1-11.

(504) Li R, Cooper C, Austin A, Livingston G. Do changes in coping style explain the effectiveness of interventions for psychological morbidity in family carers of people with dementia? A systematic review and meta-analysis. Int Psychogeriatr 2013 February;25(2):204-14.

(505) Corbett A, Stevens J, Aarsland D, Day S, Moniz-Cook E, Woods R et al. Systematic review of services providing information and/or advice to people with dementia and/or their caregivers. Int J Geriatr Psychiatry 2012 June;27(6):628-36.

(506) Sorensen S, Duberstein P, Gill D, Pinquart M. Dementia care: mental health effects, intervention strategies, and clinical implications. Lancet Neurol 2006 November;5(11):96173.

(507) Charlesworth G, Shepstone L, Wilson E, Reynolds S, Mugford M, Price D et al. Befriending carers of people with dementia: randomised controlled trial. BMJ 2008 June 7;336(7656):1295-7.

(508) Waldorff FB, Buss DV, Eckermann A, Rasmussen ML, Keiding N, Rishoj S et al. Efficacy of psychosocial intervention in patients with mild Alzheimer's disease: the multicentre, rater blinded, randomised Danish Alzheimer Intervention Study (DAISY). BMJ 2012;345:e4693.

(509) Koivisto AM, Hallikainen I, Valimaki T, Hongisto K, Hiltunen A, Karppi P et al. Early psychosocial intervention does not delay institutionalization in persons with mild Alzheimer disease and has impact on neither disease progression nor caregivers' wellbeing: ALSOVA 3-year follow-up. Int J Geriatr Psychiatry 2016 March;31(3):273-83.

(510) Charlesworth G, Burnell K, Crellin N, Hoare Z, Hoe J, Knapp M et al. Peer support and reminiscence therapy for people with dementia and their family carers: a factorial pragmatic randomised trial. J Neurol Neurosurg Psychiatry 2016.

(511) Mittelman MS, Roth DL, Coon DW, Haley WE. Sustained benefit of supportive intervention for depressive symptoms in caregivers of patients with Alzheimer's disease. Am J Psychiatry 2004 May;161(5):850-6.

(512) Joling KJ, van Marwijk HW, Smit F, van der Horst HE, Scheltens P, van de Ven PM et al. Does a family meetings intervention prevent depression and anxiety in family caregivers of dementia patients? A randomized trial. PLoS One 2012;7(1):e30936.

(513) Livingston G, Barber J, Rapaport P, Knapp M, Griffin M, King D et al. Long-term clinical and cost-effectiveness of psychological intervention for family carers of people with dementia: a single-blind, randomised, controlled trial. Lancet Psychiatry 2014 December;1(7):539-48.

(514) Livingston G, Barber J, Rapaport P, Knapp M, Griffin M, Romeo R et al. START (STrAtegies for RelaTives) study: a pragmatic randomised controlled trial to determine the clinical effectiveness and cost-effectiveness of a manual-based coping strategy programme in promoting the mental health of carers of people with dementia. Health Technology Assessment 2014 October;18(61):VII-241.

(515) Knapp M, King D, Romeo R, Schehl B, Barber J, Griffin M et al. Cost effectiveness of a manual based coping strategy programme in promoting the mental health of family carers 
of people with dementia (the START (STrAtegies for RelaTives) study): a pragmatic randomised controlled trial. Bmj-British Medical Journal 2013;347.

(516) Sommerlad A, Manela M, Cooper C, Rapaport P, Livingston G. START (STrAtegies for RelaTives) coping strategy for family carers of adults with dementia: qualitative study of participants' views about the intervention. BMJ open 2014;4(6):e005273.

(517) Nichols LO, Chang C, Lummus A, Burns R, Martindale-Adams J, Graney MJ et al. The costeffectiveness of a behavior intervention with caregivers of patients with Alzheimer's disease. J Am Geriatr Soc 2008 March;56(3):413-20.

(518) Department of Health. 'No Secrets' Guidance on developing and implementing multiagency policies and procedures to protect vulnerable adults from abuse. 2000.

(519) Cooper C, Selwood A, Blanchard M, Walker Z, Blizard R, Livingston G. Abuse of people with dementia by family carers: representative cross sectional survey. British Medical Journal 2009 January 22;338.

(520) Cooper C, Selwood A, Blanchard M, Livingston G. Abusive behaviour experienced by family carers from people with dementia: the CARD (caring for relatives with dementia) study. J Neurol Neurosurg Psychiatry 2010 June;81(6):592-6.

(521) Cooper C, Blanchard M, Selwood A, Walker Z, Livingston G. Family carers' distress and abusive behaviour: Iongitudinal study. Br J Psychiatry 2010 June;196(6):480-5.

(522) Cooper C, Katona C, Finne-Soveri H, Topinkova E, Carpenter Gl, Livingston G. Indicators of elder abuse: A crossnational comparison of psychiatric morbidity and other determinants in the Ad-HOC study. American Journal of Geriatric Psychiatry 2006 June;14(6):489-97.

(523) Natan MB, Lowenstein A, Eisikovits Z. Psycho-social factors affecting elders' maltreatment in long-term care facilities. Int Nurs Rev 2010 March;57(1):113-20.

(524) Cooper C, Dow B, Hay S, Livingston D, Livingston G. Care workers' abusive behaviour to residents in care homes: a qualitative study of types of abuse, barriers and facilitators to good care and development of an instrument for reporting of abuse anonymously. Int Psychogeriatr. In press 2012.

(525) Cooper C, Selwood A, Livingston G. The prevalence of elder abuse and neglect: a systematic review. Age Ageing 2008 March;37(2):151-60.

(526) Cooper C, Selwood A, Livingston G. Knowledge, detection and reporting of abuse by health and social care professionals: a systematic review. American Journal of Geriatric Psychiatry 2009;17(10):826-38.

(527) Kitchen G, Richardson B, Livingston G. Are nurses equipped to manage actual or suspected elder abuse? Professional Nurse 17[11], 647-650. 2002.

Ref Type: Journal (Full)

(528) Cooper C, Huzzey L, Livingston G. The effect of an educational intervention on junior doctors' knowledge and practice in detecting and managing elder abuse

COOPER2012. Int Psychogeriatr 2012 September;24(9):1447-53. 
(529) Richardson B, Kitchen G, Livingston G. The effect of education on knowledge and management of elder abuse: a randomized controlled trial. Age and Ageing 2002 September;31(5):335-41.

(530) Ayalon L, Lev S, Green O, Nevo U. A systematic review and meta-analysis of interventions designed to prevent or stop elder maltreatment. Age and Ageing 2016 March;45(2):21627.

(531) Steinert T, Noorthoorn EO, Mulder CL. The use of coercive interventions in mental health care in Germany and the Netherlands. A comparison of the developments in two neighboring countries. Frontiers in Public Health 2014;Available from: URL: http://journal.frontiersin.org/article/10.3389/fpubh.2014.00141/full\#B37

(532) Cooper C, Barber J, Griffin M, Rapaport P, Livingston G. Effectiveness of START psychological intervention in reducing abuse by dementia family carers: randomised controlled trial. International Psychogeriatrics 2016;28(6):881-7.

(533) Tschanz JT, Corcoran CD, Schwartz S, Treiber K, Green RC, Norton MC et al. Progression of cognitive, functional, and neuropsychiatric symptom domains in a population cohort with Alzheimer dementia: the Cache County Dementia Progression study. Am J Geriatr Psychiatry 2011 June;19(6):532-42.

(534) Xie J, Brayne C, Matthews FE. Survival times in people with dementia: analysis from population based cohort study with 14 year follow-up. British Medical Journal 2008 February 2;336(7638):258-62.

(535) Weuve J, Hebert LE, Scherr PA, Evans DA. Deaths in the United States among persons with Alzheimer's disease (2010-2050). Alzheimer's \& dementia 10[2], e40-46. 2016.

Ref Type: Generic

(536) Sampson EL, Gould V, Lee D, Blanchard MR. Differences in care received by patients with and without dementia who died during acute hospital admission: a retrospective case note study. Age and Ageing 2006 March;35(2):187-9.

(537) Morrison RS, Siu AL. A comparison of pain and its treatment in advanced dementia and cognitively intact patients with hip fracture. Journal of Pain and Symptom Management 2000 April;19(4):240-8.

(538) McCarthy M, AddingtonHall J, Altmann D. The experience of dying with dementia: A retrospective study. Int J Geriatr Psychiatry 1997 March;12(3):404-9.

(539) van der Steen JT, Albers G, Licht-Strunk E, Muller MT, Ribbe MW. A validated risk score to estimate mortality risk in patients with dementia and pneumonia: barriers to clinical impact. International Psychogeriatrics 2011 February;23(1):31-43.

(540) Barclay S, Froggatt K, Crang C, Mathie E, Handley M, lliffe S et al. Living in uncertain times: trajectories to death in residential care homes. British Journal of General Practice 2014 September;64(626):E576-E583.

(541) Jayes RL, Arnold RM, Fromme EK. Does this dementia patient meet the prognosis eligibility requirements for hospice enrollment? J Pain Symptom Manage 2012 November;44(5):7506. 
(542) Davies E, Higginson IJ. Better pallative care for older people. WHO Report 2004.

(543) van der Steen JT, Radbruch L, Hertogh CMPM, de Boer ME, Hughes JC, Larkin P et al. White paper defining optimal palliative care in older people with dementia: A Delphi study and recommendations from the European Association for Palliative Care. Palliative Medicine 2014 March;28(3):197-209.

(544) Chan D, Livingston G, Jones L, Sampson EL. Grief reactions in dementia carers: a systematic review. Int J Geriatr Psychiatry 2013 January;28(1):1-17.

(545) Lawrence V, Samsi K, Murray J, Harari D, Banerjee S. Dying well with dementia: qualitative examination of end-of-life care. Br J Psychiatry 2011 November;199(5):417-22.

(546) Kupeli N, Leavey G, Moore K, Harrington J, Lord K, King M et al. Context, mechanisms and outcomes in end of life care for people with advanced dementia. BMC Palliat Care 2016;15:31.

(547) Shega JW, Hougham GW, Stocking CB, Cox-Hayley D, Sachs GA. Management of noncancer pain in community-dwelling persons with dementia. Journal of the American Geriatrics Society 2006 December;54(12):1892-7.

(548) Mitchell SL, Kiely DK, Hamel MB. Dying with advanced dementia in the nursing home. Archives of Internal Medicine 2016;164(3):321-6.

(549) van der Steen JT, Meuleman-Peperkamp I, Ribbe MW. Trends in Treatment of Pneumonia among Dutch Nursing Home Patients with Dementia. Journal of Palliative Medicine 2009 September;12(9):789-95.

(550) Volicer L, Frijters DHM, van der Steen JT. Relationship between symptoms of depression and agitation in nursing home residents with dementia. Int J Geriatr Psychiatry 2012 July;27(7):749-54.

(551) Lichtner V, Dowding D, Esterhuizen P, Closs SJ, Long AF, Corbett A et al. Pain assessment for people with dementia: a systematic review of systematic reviews of pain assessment tools. Bmc Geriatrics 2014 December 17;14.

(552) Jordan A, Regnard C, O'Brien JT, Hughes JC. Pain and distress in advanced dementia: Choosing the right tools for the job. Palliative Medicine 2012 October;26(7):873-8.

(553) Cohen-Mansfield J, Thein K, Marx MS, Dakheel-Ali M, Jensen B. Sources of discomfort in persons with dementia. JAMA Intern Med 2013 July 22;173(14):1378-9.

(554) Sampson EL, Candy B, Jones L. Enteral tube feeding for older people with advanced dementia. Cochrane Database Syst Rev 2009;(2):CD007209.

(555) Palecek EJ, Teno JM, Casarett DJ, Hanson LC, Rhodes RL, Mitchell SL. Comfort feeding only: a proposal to bring clarity to decision-making regarding difficulty with eating for persons with advanced dementia. J Am Geriatr Soc 2010 March;58(3):580-4.

(556) Bentur N, Sternberg S, Shuldiner J, Dwolatzky T. Feeding tubes for older people with advanced dementia living in the community in Israel. Am J Alzheimers Dis Other Demen 2015 March;30(2):165-72. 
(557) Wada T, Imai H, Fukutomi E, Chen WL, Okumiya K, Ishimoto $Y$ et al. Preferred feeding methods for dysphagia due to end-stage dementia in community-dwelling elderly people in Japan. J Am Geriatr Soc 2014 September;62(9):1810-1.

(558) Ahronheim JC, Mulvihill M, Sieger C, Park P, Fries BE. State practice variations in the use of tube feeding for nursing home residents with severe cognitive impairment. J Am Geriatr Soc 2001 February;49(2):148-52.

(559) Livingston G, Lewis-Holmes E, Pitfield C, Manela M, Chan D, Constant E et al. Improving the end-of-life for people with dementia living in a care home: an intervention study. Int Psychogeriatr 2013 November;25(11):1849-58.

(560) Arcand M, Monette J, Monette M, Sourial N, Fournier L, Gore B et al. Educating nursing home staff about the progression of dementia and the comfort care option: impact on family satisfaction with end-of-life care. J Am Med Dir Assoc 2009 January;10(1):50-5.

(561) Sampson EL, Ritchie CW, Lai R, Raven PW, Blanchard MR. A systematic review of the scientific evidence for the efficacy of a palliative care approach in advanced dementia. International Psychogeriatrics 2005 March;17(1):31-40.

(562) Dening KH, Jones L, Sampson EL. Preferences for end-of-life care: A nominal group study of people with dementia and their family carers. Palliative Medicine 2013 May;27(5):409-17.

(563) Robinson L, Dickinson C, Bamford C, Clark A, Hughes J, Exley C. A qualitative study: Professionals' experiences of advance care planning in dementia and palliative care, 'a good idea in theory but ...'. Palliative Medicine 2013 May;27(5):401-8.

(564) Sampson EL, Thuné-Boyle I, Kukkastenvehmas R, Jones L, Tookman A, King M. Palliative care in advanced dementia: A mixed methods approach for the development of a complex intervention. BMC Palliative Care 2008;7:8.

(565) Tomlinson E, Spector A, Nurock S, Stott J. Euthanasia and physician-assisted suicide in dementia: A qualitative study of the views of former dementia carers. Palliative Medicine 2015 September;29(8):720-6.

(566) Kaplan DB, Berkman B. Dementia care: A global concern and social work challenge. International Social Work 2011 May;54(3):361-73.

(567) Kaplan DB, Andersen TC. The transformative potential of social work's evolving practice in dementia care. Journal of gerontological social work 2013;56:164-76.

(568) Somme D, Trouve H, Drame M, Gagnon D, Couturier Y, Saint-Jean O. Analysis of case management programs for patients with dementia: a systematic review. Alzheimers Dement 2012 September;8(5):426-36.

(569) Bodenheimer T, Wagner EH, Grumbach K. Improving primary care for patients with chronic illness: the chronic care model, Part 2. Journal of the American Medical Disorders Association 2002;288:1909-14.

(570) Roett, MA, Coleman MT. Practice improvement, part II: collaborative practice and teambased care. FP Essent 2013;414:11-8. 
(571) Bossen AL, Specht JKP, McKenzie SE. Needs of People with Early-Stage Alzheimer's Disease REVIEWING THE EVIDENCE. Journal of Gerontological Nursing 2009 March;35(3):8-15.

(572) Galvin JE, Valois L, Zweig Y. Collaborative transdisciplinary team approach for dementia care. Neurodener Dis Manag 2014;4:455-69.

(573) Kodner DL. All together now: a conceptual exploration of integrated care. Healthc Q 2009;13:6-15.

(574) Lim J, Goh J, Chionh HL, Yap P. Why do patients and their families not use services for dementia? Perspectives from a developed Asian country. International Psychogeriatrics 2012 October;24(10):1571-80.

(575) Nelson T, Livingston G, Knapp M, Manela M, Kitchen G, Katona C. Slicing the health service cake: the Islington study. Age Ageing 2002 November;31(6):445-50.

(576) Nelson T, Fernandez JL, Livingston G, Knapp M, Katona C. Does diagnosis determine delivery? The Islington study of older people's needs and health care costs. Psychol Med 2004 January;34(1):147-55.

(577) Warshaw GA, Bragg EJ. Preparing The Health Care Workforce To Care For Adults With Alzheimer's Disease And Related Dementias. Health Affairs 2014 April;33(4):633-41.

(578) Pimouguet C, Lavaud T, Dartigues JF, Helmer C. Dementia case management effectiveness on health care costs and resource utilization: a systematic review of randomized controlled trials. J Nutr Health Aging 2010 October;14(8):669-76.

(579) Reilly S, Miranda-Castillo C, Malouf R, Hoe J, Toot S, Challis D et al. Case management approaches to home support for people with dementia. Cochrane Database Syst Rev 2015;1:CD008345.

(580) Hickam, D. H., Weiss, J. W., Guise, J. M., Buckley, D., Motu'apuaka, M., Graham, E., Wasson, N., and Saha, S. Outpatient Case Management for Adults with Medical Illness and Complex Care Needs. Comparative Effectiveness Review No. 99. 2013 Jan.

(581) Tam-Tham H, Cepoiu-Martin C, Ronksley PE. Dementia case management and risk of longterm care placement: a systematic review and meta-analysis. International Journal of Geriatric Psychiatry 8[5], 426-436. 2012.

Ref Type: Generic

(582) Samus QM, Johnston D, Black BS, Hess E, Lyman C, Vavilikolanu A et al. A multidimensional home-based care coordination intervention for elders with memory disorders: the maximizing independence at home (MIND) pilot randomized trial. Am J Geriatr Psychiatry 2014 April;22(4):398-414.

(583) Callahan CM, Boustani MA, Unverzaqt FW, Austrom MG, Damush TM, Perkins AJ et al. Effectiveness of collaborative care for older adults with Alzheimer disease in primary care: a randomized controlled trial. JAMA 2006 May 10;10:2148-57.

(584) Amjad H, Carmichael D, Austin AM, Chang CH, Bynum JP. Continuity of Care and Health Care Utilization in Older Adults With Dementia in Fee-for-Service Medicare. JAMA Intern Med 2016 July 25. 
(585) Brown AF, Vassar SD, Connor KI, Vickrey BG. Collaborative Care Management Reduces Disparities in Dementia Care Quality for Caregivers with Less Education. Journal of the American Geriatrics Society 2013 February;61(2):243-51.

(586) Gitlin LN, Winter L, Dennis MP, Hodgson N, Hauck WW. A biobehavioral home-based intervention and the well-being of patients with dementia and their caregivers: the COPE randomized trial. JAMA 2010 September 1;304(9):983-91.

(587) Lai C, Yeung J, Mok V, Chi I. Special care units for dementia individuals with behavioural problems. Cochrane Database of Systematic Reviews 9 A.D. July 10;4:CD006470. doi: 10.1002/14651858.CD006470.pub2.

(588) Gaugler JE, Yu F, Krichbaum K, Wyman JF. Predictors of Nursing Home Admission for Persons with Dementia. Medical Care 2009 February;47(2):191-8.

(589) Luppa M, Luck T, Weyerer S, Konig HH, Brahler E, Riedel-Heller SG. Prediction of institutionalization in the elderly. A systematic review. Age and Ageing 2010 January;39(1):31-8.

(590) Matthews FE, Dening T. Prevalence of dementia in institutional care. Lancet 2002 July 20;360(9328):225-6.

(591) Bernstein AB, Remsburg RE. Estimated prevalence of people with cognitive impairment: results from nationally representative community and institutional surveys. Gerontologist 2007 June;47:350-4.

(592) Stewart R, Hotopf M, Dewey M, Ballard C, Bisla J, Calem M et al. Current prevalence of dementia, depression and behavioural problems in the older adult care home sector: the South East London Care Home Survey. Age and Ageing 2014 July;43(4):562-7.

(593) Seitz D, Purandare N, Conn D. Prevalence of psychiatric disorders among older adults in long-term care homes: a systematic review. International Psychogeriatrics 2010 November;22(7):1025-39.

(594) Centers for Medicare \& Medicaid Services. Guide to choosing a nursing home. Department of Health and Human Services Publication No. CMS-02174. 2002. Baltimore, MD: US, Available at: http://www.feddesk.com/freehandbooks/1216-4.pdf Exit Disclaimer.

Ref Type: Generic

(595) Zimmerman S, Anderson WL, Brode S, Jonas D, Lux L, Beeber AS et al. Systematic Review: Effective Characteristics of Nursing Homes and Other Residential Long-Term Care Settings for People with Dementia. Journal of the American Geriatrics Society 2013 August;61(8):1399-409.

(596) Hughes CM, Lapane K, Watson MC, Davies HT. Does organisational culture influence prescribing in care homes for older people? A new direction for research. Drugs Aging 2007;24(2):81-93.

(597) Chen Y, Briesacher BA, Field TS, Tjia J, Lau DT, Gurwitz JH. Unexplained variation across US nursing homes in antipsychotic prescribing rates. Arch Intern Med 2010 January 11;170(1):89-95. 
(598) Beerens H, Zwakhalen S, Verbeek H, Ruwaard D, Hamers JP. Factors Associated with Quality of Life of People with Dementia in Long-Term Care Facilities. Gerontologist 2013 November;53:417.

(599) Moyle W, O'Dwyer S. Quality of life in people living with dementia in nursing homes. Current Opinion in Psychiatry 2016;2012:480-4.

(600) Beerens HC, Sutcliffe C, Renom-Guiteras A, Soto ME, Suhonen R, Zabalequi A. Quality of life and quality of care for people with dementia receiving long term insitutional care or professional home care: the European RightTimePlaceCare study. Journal of the American Medical Disorders Association 2014;15:54-61.

(601) Jutkowitz E, Brasure M, Fuchs E, Shippee T, Kane RA, Fink HA et al. Care-Delivery Interventions to Manage Agitation and Aggression in Dementia Nursing Home and Assisted Living Residents: A Systematic Review and Meta-analysis. J Am Geriatr Soc 2016 March;64(3):477-88.

(602) Rokstad AM, Doble BS, Engedal K, Kirkevold O, Benth JS, Selbaek G. The impact of the Dementia $A B C$ educational programme on competence in person-centred dementia care and job satisfaction of care staff. Int J Older People Nurs 2016 November 20.

(603) Teri L, McKenzie G, Logsdon RG, McCurry SM, Bollin S, Mead J et al. Translation of two evidence-based programs for training families to improve care of persons with dementia. Gerontologist 2012 August;52(4):452-9.

(604) Teri L, McKenzie GL, Pike KC, Farran CJ, Beck C, Paun O et al. Staff training in assisted living: evaluating treatment fidelity. Am J Geriatr Psychiatry 2010 June;18(6):502-9.

(605) Ballard C, Corbett A, Howard R. Prescription of antipsychotics in people with dementia. Br J Psychiatry 2014 July;205(1):4-5.

(606) Thompson Coon J, Abbott R, Rogers M, Whear R, Pearsons S, Lang I. Interventions to reduce inappropriate prescribing of antipsychotic medications in people with dementia resident in care homes: a systematic review. Journal of the American Medical Disorders Association 2014;15:706-18.

(607) Ballard C, Orrell M, YongZhong S, Moniz-Cook E, Stafford J, Whittaker R et al. Impact of Antipsychotic Review and Nonpharmacological Intervention on Antipsychotic Use, Neuropsychiatric Symptoms, and Mortality in People With Dementia Living in Nursing Homes: A Factorial Cluster-Randomized Controlled Trial by the Well-Being and Health for People With Dementia (WHELD) Program. Am J Psychiatry 2016 March 1;173(3):252-62.

(608) Rapaport P, Livingston G, Mulla A, Murray J, Cooper C. A systematic review of the effective components of psychosocial interventions delivered by care home staff to people with dementia. BMJ open. In press 2017.

(609) Shah F, Burack O, Boockvar KS. Perceived barriers to communication between hospital and nursing home at time of patient transfer. J Am Med Dir Assoc 2010 May;11(4):239-45.

(610) Livingston G, Pitfield C, Morris J, Manela M, Lewis-Holmes E, Jacobs H. Care at the end of life for people with dementia living in a care home: a qualitative study of staff experience and attitudes. Int J Geriatr Psychiatry 2012 June;27(6):643-50. 
(611) Lester P, Stefanacci RG, Chen DG. Nursing home procedures on transitions of care. J Am Med Dir Assoc 2009 November;10(9):634-8.

(612) Anderson RA, Issel LM, McDaniel Jr RR. Nursing homes as complex adaptive systems: relationship between management practice and resident outcomes. Nurs Res 2003 January;52(1):12-21.

(613) Goodman C, Dening T, Gordon AL, Davies SL, Meyer J, Martin FC et al. Effective health care for older people living and dying in care homes: a realist review. BMC Health Serv Res 2016;16(1):269.

(614) McConnell ES, Karel MJ. Improving Management of Behavioral and Psychological Symptoms of Dementia in Acute Care: Evidence and Lessons Learned From Across the Care Spectrum. Nurs Adm Q 2016 July;40(3):244-54.

(615) Karantzas GC, Mellor D, McCabe MP, Davison TE, Beaton P, Mrkic D. Intentions to quit work among care staff working in the aged care sector. Gerontologist 2012 August;52(4):506-16.

(616) Karantzas GC, McCabe MP, Mellor D, Von TK, Davison TE, O'Connor D et al. Organizational climate and self-efficacy as predictors of staff strain in caring for dementia residents: A mediation model. Arch Gerontol Geriatr 2016 September;66:89-94.

(617) Feldman PH, Kane RL. Strengthening research to improve the practice and management of long-term care. Milbank Q 2003;81(2):179-220, 171.

(618) Rosenblatt A, Samus QM, Steele CD, Baker AS, Harper MG, Brandt J et al. The Maryland Assisted Living Study: prevalence, recognition, and treatment of dementia and other psychiatric disorders in the assisted living population of central Maryland. J Am Geriatr Soc 2004 October;52(10):1618-25.

(619) Kopetz S, Steele CD, Brandt J, Baker A, Kronberg M, Galik E et al. Characteristics and outcomes of dementia residents in an assisted living facility. Int J Geriatr Psychiatry 2000 July;15(7):586-93.

(620) Smith M, Buckwalter KC, Kang H, Ellingrod V, Schultz SK. Dementia-specific assisted living: clinical factors and psychotropic medication use. J Am Psychiatr Nurses Assoc 2008 March;14(1):39-49.

(621) Smith M, Buckwalter KC, Kang H, Ellingrod V, Schultz SK. Dementia care in assisted living: needs and challenges. Issues Ment Health Nurs 2008 August;29(8):817-38.

(622) Tolson D, Rolland Y, Andrieu S, Aquino JP, Beard J, Benetos A et al. International Association of Gerontology and Geriatrics: a global agenda for clinical research and quality of care in nursing homes. J Am Med Dir Assoc 2011 March;12(3):184-9.

(623) Verbeek H, van RE, Zwakhalen SM, Kempen GI, Hamers JP. Small, homelike care environments for older people with dementia: a literature review. Int Psychogeriatr 2009 April;21(2):252-64.

(624) Ausserhofer D, Deschodt M, De GS, van AT, Meyer G, Verbeek H et al. "There's No Place Like Home": A Scoping Review on the Impact of Homelike Residential Care Models on 
Resident-, Family-, and Staff-Related Outcomes. J Am Med Dir Assoc 2016 August 1;17(8):685-93.

(625) Annerstedt L. An attempt to determine the impact of group living care in comparison to traditional long-term care on demented elderly patients. Aging (Milano ) 1994 October;6(5):372-80.

(626) Te BS, Pot AM, Depla M, Smit D, de LJ, Eefsting J. Group living homes for older people with dementia: the effects on psychological distress of informal caregivers. Aging Ment Health 2008 November;12(6):761-8.

(627) Nakanishi M, Nakashima T, Sawamura K. Quality of life of residents with dementia in a group-living situation: an approach to creating small, homelike environments in traditional nursing homes in Japan. Nihon Koshu Eisei Zasshi 2012 January;59(1):3-10.

(628) Carrillo MC, Dishman E, Plowman T. Everyday technologies for Alzheimer's disease care: Research findings, directions, and challenges. Alzheimers \& Dementia 2009 November;5(6):479-88.

(629) Burstein AA, DaDalt O, Kramer B, D'Ambrosio L, Coughlin J. Technology and the caregiving dilemma: A comparative look at awareness, attitudes, and the role of technology. Alzheimer's \& Dementia: The Journal of the Alzheimer's Association 2014;10(4):P571.

(630) Bossen AL, Kim H, Williams KN, Steinhoff AE, Strieker M. Emerging roles for telemedicine and smart technologies in dementia care. Smart homecare technology and telehealth 2015;3:49.

(631) Bharucha AJ, Anand V, Forlizzi J, Dew MA, Reynolds CF, Stevens S et al. Intelligent Assistive Technology Applications to Dementia Care: Current Capabilities, Limitations, and Future Challenges. American Journal of Geriatric Psychiatry 2009 February;17(2):88-104.

(632) Robinson H, Macdonald B, Kerse N, Broadbent E. The psychosocial effects of a companion robot: a randomized controlled trial. J Am Med Dir Assoc 2013 September;14(9):661-7.

(633) Jøranson N, .Pedersen I, Ihlebæk C, Rokstad AM. Effects on symptoms of agitation and depression in persons with dementia participating in robot-assisted activity: A clusterrandomized controlled trial. J Am Med Dir Assoc 2016;16:867-73.

(634) Bharucha AJ, Anand V, Forlizzi J, Dew MA, Reynolds CF, Stevens S et al. Intelligent Assistive Technology Applications to Dementia Care: Current Capabilities, Limitations, and Future Challenges. American Journal of Geriatric Psychiatry 2009 February;17(2):88-104.

(635) Godwin KM, Mills WL, Anderson JA, Kunik ME. Technology-Driven Interventions for Caregivers of Persons With Dementia: A Systematic Review. American Journal of Alzheimer's Disease and Other Dementias 2013;28(3):216-22.

(636) Finkel S, Czaja SJ, Schulz R, Martinovich Z, Harris C, Pezzuto D. E-care: A telecommunications technology intervention for family caregivers of dementia patients. American Journal of Geriatric Psychiatry 2007 May;15(5):443-8.

(637) Connelly K, Laghari KUR, Mokhtari M, Falk TH. Approaches to Understanding the Impact of Technologies for Aging in Place: A Mini-Review. Gerontology 2014;60(3):282-8. 
(638) Sharkey N, Sharkey A. The Eldercare Factory. Gerontology 2012;58(3):282-8. 